\title{
Étude thermique globale du Rhône - Impacts hydrobiologiques des échauffements cumulés
}

\section{Global thermal study of the Rhone - Hydrobiological impact of cumulative warming}

\author{
M. Khalanski(1), G. Carrel(2) ${ }^{(2)}$ B. Desaint ${ }^{(3)}$, J.-F. Fruget( ${ }^{(4)}$, J.-M. Olivier(5), \\ A. Poirel(3), Y. Souchon ${ }^{(6)}$ \\ (1) Comité d'hydroécologie, Laboratoire National d'Hydraulique et Environnement, 6 quai Watier, BP 49, \\ 78401 Chatou Cedex, France \\ (2) Cemagref Aix-en-Provence, U.R. Hydrobiologie, 3275 route de Cézanne, CS 40061, \\ 13182 Aix-en-Provence Cedex 5, France \\ (3) EDF Division Technique Générale, Service Environnement Aquatique, 21 avenue de l'Europe, BP 41, \\ 38040 Grenoble Cedex, France \\ (4) ARALEP / Écologie des eaux des eaux douces, Domaine Scientifique de la Doua, Bâtiment CEI 66, \\ boulevard Niels Bohr, BP 2132, 69603 Villeurbanne Cedex, France \\ (5) Université Claude Bernard-Lyon 1, UMR CNRS 5023, Écologie des hydrosystèmes fluviaux, \\ 43 boulevard du 11 novembre 1918, 69622 Villeurbanne Cedex, France \\ (6) Cemagref Lyon, Laboratoire d'Hydroécologie Quantitative, 3 bis quai Chauveau, CP 220, \\ 69336 Lyon Cedex 09, France \\ e-mail : cecile.delattre@edf.fr
}

Résumé - L'étude thermique globale du Rhône, lancée en 2000 à l'initiative de la DRIRE Rhône-Alpes en partenariat avec la DIREN, l'agence de l'eau RM\&C, le Service de la Navigation et EDF, a pour objectif de déterminer l'incidence des rejets de chaleur cumulés sur le régime thermique du fleuve et ses conséquences hydrobiologiques. Après une caractérisation du régime thermique du Rhône (phase 1), puis une évaluation de l'échauffement apporté par les centres nucléaires de production d'électricité (CNPE) au cours de la phase 2, la phase 3 concerne l'impact hydrobiologique de ces échauffements cumulés. Cet article reprend, avec quelques éléments nouveaux, le rapport de synthèse de la phase 3 paru en avril 2006. Le Rhône est un fleuve totalement aménagé pour la production hydro-électrique et la navigation. Ces aménagements ont modifié les conditions hydro-morphologiques originelles et créé de nouveaux milieux (canaux, retenues, sections court-circuitées à débit régulé). À ces facteurs hydro-morphologiques contemporains qui structurent l'habitat physique des espèces, se superposent depuis la fin des années 70 des changements de la qualité chimique de l'eau (réduction de la pollution organique et de certaines pollutions toxiques). Par ailleurs, l'apparition et le développement d'espèces exogènes modifient la structure biologique et le fonctionnement de l'écosystème rhodanien. Au cours des trois dernières décennies, le régime thermique du Rhône a été affecté par le changement climatique. II s'est traduit par un échauffement moyen d'environ $1,5^{\circ} \mathrm{C}$ sur le Haut-Rhône et de $3,0^{\circ} \mathrm{C}$ sur le Bas-Rhône. Dans cette dérive générale des températures, sur les quinze dernières années les échauffements résiduels moyens cumulés liés 
aux rejets thermiques des CNPE sont estimés à environ $0,6^{\circ} \mathrm{C}$ à l'amont de Saint-Alban et $1,4^{\circ} \mathrm{C}$ à l'amont d'Aramon. L'étude des chroniques de la surveillance hydro-écologique à long terme des CNPE indique que les modifications constatées sur les peuplements de macro-invertébrés et de poissons s'expliquent en premier lieu par les effets du changement climatique. En réponse à l'élévation de température, une modification générale dans la structure des peuplements de macro-invertébrés et de poissons a été enregistrée sur le Haut-Rhône au cours des 25 dernières années et particulièrement pendant la dernière décennie. Les espèces thermophiles et lénitophiles sont favorisées alors que les espèces sténothermes d'eau froide et rhéophiles régressent. À Bugey, ces tendances sont plus nettement visibles dans les stations exposées en permanence à des échauffements plus élevés (environ $+5^{\circ} \mathrm{C}$ et $+9^{\circ} \mathrm{C}$ ) que dans celles situées après mélange du rejet. Sur le Bas-Rhône, où les peuplements sont déjà dominés par des taxons thermophiles et lénitophiles, une dérive moins marquée est également observée. Dans les tronçons court-circuités et les annexes hydrauliques, le régime thermique est en grande partie indépendant de la température du Rhône en période estivale. Dans les conditions actuelles, ces milieux à haute valeur écologique ne sont pas affectés par les rejets thermiques des CNPE. Cette étude formule également quelques recommandations pour des ajustements des programmes de surveillance hydro-écologique des CNPE et du programme de surveillance renforcé en cas de crise climatique ainsi que sur de nouvelles actions de recherche. Dans ce domaine, il est préconisé de développer des modèles hydrodynamiques d'habitat sur les secteurs à fort enjeu écologique couplant les conditions hydrologiques et thermiques.

Mots clés - température, échauffement, Rhône, suivi long terme, macro-invertébrés, poissons, impact thermique

\begin{abstract}
The global thermal study of the Rhone which was launched in 2000 at the initiative of the Rhone-Alps DRIRE (Regional Board for Industry, Research and Environment), in partnership with the DIREN Regional Environment Office, the RM\&C water board, the Rhone Navigation Authorities and EDF, marked an attempt to determine the impact of cumulative warm water releases on the thermal regime of the river as a whole, and the resulting hydrobiological effects. Following the description of the thermal regime of the Rhone (phase 1) and an assessment of warming generated by the nuclear power plants (CNPE) on the river (phase 2), phase 3 bears on the hydrobiological impact of the cumulative warming. This article summarizes, with some updates, the report on phase 3 which was issued in April 2006. The French section of the Rhone is equipped with hydropower and navigation installations which create hydro-morphological conditions which are quite distinct, ranging from semi-natural rapid flow to reservoirs with slower flow and including canals and bypass sections with controlled discharge, in the original bed of the river. These habitat-structuring hydro-morphological factors, since the end of the Seventies, have been compounded by changes in the chemical quality of the water (a reduction in organic pollution and in certain toxic pollutants) and the development of exogenous species which influence the biological structure and the dynamics of the Rhone ecosystem. Over the last three decades, the thermal regime of the Rhone has essentially been affected by climate change, which has resulted in warming on the order of $1.5^{\circ} \mathrm{C}$ in the upper Rhone and $3.0^{\circ} \mathrm{C}$ in the lower Rhone. Within this general shift in temperature, it is estimated that the mean contribution of releases from the nuclear plants is on the order of $0.6^{\circ} \mathrm{C}$ upstream Saint-Alban and $1.4^{\circ} \mathrm{C}$ upstream Aramon. Statistical processing of long-term hydro-ecological monitoring data from the nuclear plants has shown that the changes observed in macro-invertebrate and fish stocks can first be explained by the effects of climate change. In response to the rise in temperature, a general modification in the structure of macro-invertebrate and fish stocks has been found in the upper Rhone over the last 25 years, most particularly in the
\end{abstract}


last decade: species that prefer warm water and slow flows are favored, while cold-water stenothermic and rheophilic species show a tendency to regress. These changes are markedly more visible at the downstream sites close to the Bugey plant, permanently exposed to greater warming (around $+5^{\circ} \mathrm{C}$ and $+9^{\circ} \mathrm{C}$ ) than that found further downstream after complete mixing of the power plant releases. In the lower Rhone, where stocks were already dominated by thermophilic and slow-flow taxons, the same shift is found, though it is less marked. In the bypass sections and the secondary channels, the thermal regime is largely independent of the temperature in the Rhone in summer; under present conditions, these reaches of great ecological importance are not affected by the thermal releases from the nuclear power plants. Lastly, a few recommendations are formulated. These bear on changes to be made in programs for hydro-ecological monitoring of the nuclear plants and the stepped-up monitoring program in case of climate crisis, as well as on possible research. In this latter regard, the recommendation is made to develop hydrodynamic habitat models of sectors with high ecological importance, coupling hydrological and thermal conditions.

Key words - temperature, temperature rise, Rhône river, long term monitoring, macroinvertebrates, fish, thermal impact

\section{CONTEXTE ET OBJECTIFS DE L'ÉTUDE}

Le Rhône est le fleuve européen le plus équipé pour la production électrique d'origine thermique et hydraulique (Fig. 1). Avec 14 réacteurs, les quatre centres nucléaires de production d'électricité (CNPE) actuellement en service produisent $22 \%$ de l'électricité nucléaire française et les centrales hydrauliques fournissent $26 \%$ de la production d'électricité hydroélectrique nationale (Agence de l'Eau Rhône Méditerranée \& Corse, 2005). En 2004, environ 93,5 TWh et 13,7 TWh ont été produits respectivement par les CNPE rhodaniens et les centrales hydrauliques de la Compagnie nationale du Rhône (CNR).

En dehors de leur intérêt économique, ces sources d'énergie offrent deux avantages majeurs : l'énergie nucléaire contribue à réduire les émissions industrielles de gaz à effet de serre et l'hydroélectricité représente la plus grande part des énergies renouvelables dont la progressive substitution dans la consommation nationale est imposée par la directive européenne 2001/77/CE du 27 septembre 2001 relative à la promotion de l'électricité produite à partir de sources d'énergie renouvelables (SER).

Un processus d'amélioration de la qualité de l'eau et des milieux aquatiques a été engagé sur le Rhône en vue d'atteindre un " bon état écologique » ou un "bon potentiel écologique " d'ici à 2015 au titre de la directive cadre sur l'eau (DCE) du 23 octobre 2000, transcrite en droit français par la loi du 21 avril 2004. La mise en œuvre de la directive se fait par paliers selon la programmation suivante : état des lieux du district Rhône et côtiers méditerranéens, réalisé en 2005, programme de surveillance, plan de gestion pour la fin 2009 (qui constituera une révision du SDAGE établi au titre de la loi sur l'eau de 1992), programme de mesures à définir d'ici 2009. Parmi les points marquants de la démarche définie par la 


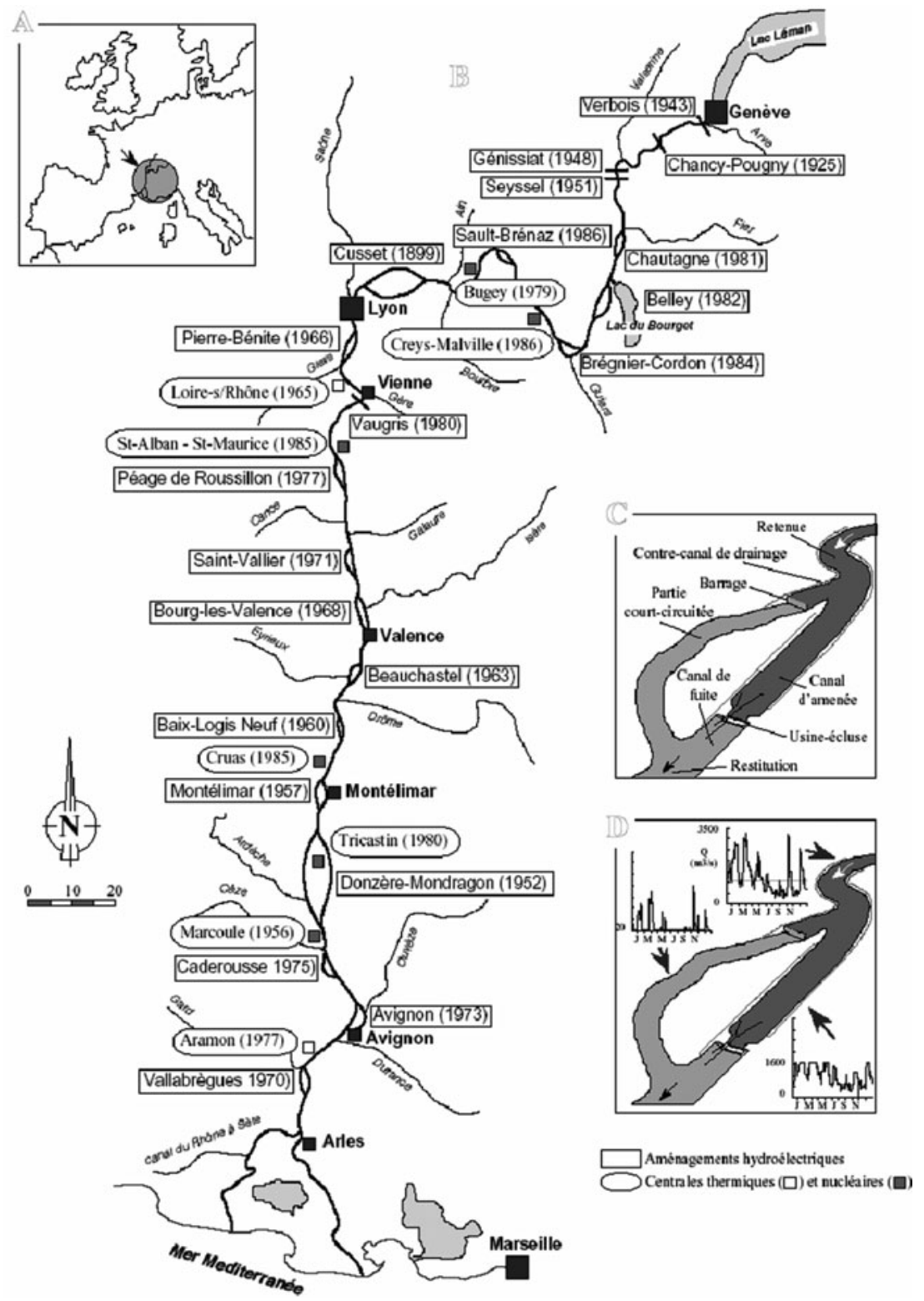

Fig. 1. Localisation des aménagements hydroélectriques et des centrales thermiques sur le Rhône de Genève à la Méditerranée (Fruget, 2003). A - Carte de localisation générale. B - Liste des ouvrages et sites industriels. C - Un aménagement hydroélectrique rhodanien type. D - Contrastes hydrologiques sur les secteurs de l'aménagement : caractéristiques des débits annuels.

Fig. 1. Location of hydroelectric dams and thermal power plants from Geneva to the Mediterranean Sea (Fruget, 2003). A - General location map. B - Dams and power plants. C - Standard hydraulic scheme on the Rhône river. D - Typical annual flow regime in the 3 parts of an hydraulic scheme. 
DCE figurent une obligation de résultats basée sur des indicateurs biologiques et chimiques, la prise en compte des contextes sociaux et économiques et la participation des acteurs de l'eau et du public.

Une des « onze questions importantes » identifiée dans la première consultation officielle relative à l'état des lieux concerne l'hydroélectricité (Comité de bassin Rhône Méditerranée \& Corse, 2004) : comment rendre compatible le développement d'une énergie renouvelable et la protection des milieux aquatiques? Le même questionnement s'applique aux centrales nucléaires de grande puissance installées sur le Rhône (Tab. I) et concerne en particulier l'incidence des rejets de chaleur cumulés sur le régime thermique du fleuve et leurs impacts environnementaux.

C'est dans cette perspective que s'inscrit l'étude thermique du Rhône, lancée en 2000 à l'initiative de la DRIRE Rhône-Alpes en partenariat avec la DIREN, l'agence de l'eau RM\&C, le service de la navigation et EDF. L'étude définie par un comité technique et suivie par un comité de pilotage, est constituée de trois étapes chronologiques:

- la phase 1 réalisée en 2000 et 2001 a donné une description du régime thermique du Rhône à

Tableau I. L'équipement thermique du Rhône depuis 1970. En 2006, les principaux rejets thermiques proviennent de trois CNPE en circuit ouvert: Bugey, Saint-Alban et Tricastin. RGG: réacteur graphitegaz, REP: réacteur à eau pressurisée, RNR: réacteur à neutrons rapides, THF : chaudière thermique à flamme. *En circuit fermé, la température du rejet dépend de la température et de l'humidité de l'air ; l'échauffement est minimum en été et maximum en hiver.

Table I. Thermal power plant equipment on the Rhone river since 1970. In 2006, the main contributors to thermal discharges are the three nuclear power plants equipped with once through cooling systems: Bugey, Saint-Alban and Tricastin. RGG: gas cooled reactor, REP: pressurized water reactor, RNR: fast neutron reactor, THF : fossil fuelled power plant. *In closed cooling water circuits, the discharge temperature depends on air temperature and humidity; the temperature rise is lowest in summer and highest in winter.

\begin{tabular}{|c|c|c|c|c|c|c|}
\hline \multirow[b]{2}{*}{ Sites } & \multirow{2}{*}{$\begin{array}{l}\text { Tranches et } \\
\text { puissance } \\
\text { électrique }\end{array}$} & \multirow{2}{*}{$\begin{array}{l}\text { Année de } \\
\text { mise en } \\
\text { service }\end{array}$} & \multirow{2}{*}{$\begin{array}{l}\text { Année } \\
\text { d'arrêt }\end{array}$} & \multicolumn{3}{|c|}{ Circuit de refroidissement } \\
\hline & & & & $\begin{array}{l}\text { Type } \\
\text { de } \\
\text { circuit }\end{array}$ & $\begin{array}{l}\text { Débit } \\
\text { rejeté } \\
\left(\mathrm{m}^{3} / \mathrm{s}\right)\end{array}$ & $\begin{array}{l}\text { Échauffement } \\
\text { maximum } \\
\text { dans l'ouvrage } \\
\text { de rejet }\end{array}$ \\
\hline Creys-Malville (RNR) & $1 \times 1200 \mathrm{MWe}$ & 1986 & 1998 & ouvert & 38 & $12^{\circ} \mathrm{C}$ \\
\hline Bugey 1 (RGG) & $1 \times 540 \mathrm{MWe}$ & 1972 & 1994 & ouvert & 31 & $10^{\circ} \mathrm{C}$ \\
\hline CNPE Bugey (REP) 2 \& 3 & $2 \times 900 \mathrm{MWe}$ & 1979 & & ouvert & $2 \times 46$ & $10^{\circ} \mathrm{C}$ \\
\hline $4 \& 5$ & $2 \times 900 \mathrm{MWe}$ & $1979-1980$ & & fermé & 11 & variable* \\
\hline Loire-sur-Rhône (THF) & $4 \times 250 \mathrm{MWe}$ & $1965-1971$ & 2005 & ouvert & $4 \times(7-9)$ & $12^{\circ} \mathrm{C}$ \\
\hline CNPE Saint-Aban (REP) & $2 \times 1300 \mathrm{MWe}$ & $1986-1987$ & & ouvert & $2 \times 64$ & $10^{\circ} \mathrm{C}$ \\
\hline CNPE Cruas (REP) & $4 \times 900 \mathrm{MWe}$ & 1984-1985 & & fermé & $4 \times 4$ & variable* \\
\hline CNPE Tricastin (REP) & $4 \times 900 \mathrm{MWe}$ & $1980-1981$ & & ouvert & $4 \times 47$ & $11^{\circ} \mathrm{C}$ \\
\hline Marcoule (RNR) & $1 \times 250 \mathrm{MWe}$ & 1973 & & ouvert & 10 & $7^{\circ} \mathrm{C}$ \\
\hline Aramon (THF) & $1 \times 700 \mathrm{MWe}$ & 1977 & & ouvert & 36 & $11,5^{\circ} \mathrm{C}$ \\
\hline
\end{tabular}


partir de données collectées en 15 stations de mesures sur la période 1977-1999 (Lauters \& Poirel, 2001a, 2001b);

- la phase 2, achevée en 2004 (Desaint, 2004) a évalué l'échauffement cumulé de l'amont vers l'aval apporté par les CNPE et actualisé les données de la phase initiale jusqu'en 2003;

- la phase 3, achevée en 2006, a réalisé une synthèse des suivis environnementaux des 4 CNPE.

Au cours de l'étude, l'épisode caniculaire de 2003 a été l'occasion de renforcer le recueil des données et de procéder à une première synthèse des connaissances disponibles en qualité d'eau et en hydrobiologie sous l'égide de la DIREN (DIREN de Bassin RMC, 2003).

Les équipes de chercheurs ayant réalisé la phase 3 (Cemagref Lyon et Aix-en-Provence, Université Lyon 1, ARALEP) ont analysé les chroniques de données disponibles sur le Rhône afin d'identifier et de caractériser :

- les descripteurs du régime thermique du fleuve ayant une pertinence biologique (Carrel, 2006a),

- la qualité physico-chimique de l'eau (Carrel, 2006b),

- les peuplements d'invertébrés et de plantes aquatiques (Bady \& Fruget, 2006 ; Fruget, 2006),

- les peuplements de poissons (Daufresne et al., 2005; Souchon et al., 2005; Olivier \& Carrel, 2006).

Ce travail décrit les évolutions spatiales et temporelles sur 25 à 30 ans en relation avec la composante naturelle de la température du fleuve et les échauffements cumulés des rejets thermiques des CNPE.
La synthèse de la phase 3 parue en avril 2006 et faisant l'objet de cet article présente les principaux éléments issus de ces rapports, complétés par des informations permettant de mettre en perspective ces données au regard des grands objectifs d'amélioration de la qualité écologique du fleuve.

\section{LES PRINCIPAUX FACTEURS DE CHANGEMENT DES PEUPLEMENTS DU RHÔNE}

\subsection{Facteurs hydro-morphologiques}

Les aménagements hydro-électriques et de navigation du Rhône ont créé des conditions hydro-morphologiques contrastées associant des sections à écoulement semi-rapide, des retenues à écoulement ralenti, des canaux et des tronçons court-circuités à débit régulé.

Au début du $19^{\mathrm{e}}$ siècle le Rhône, comme le Rhin, était un fleuve dont le lit essentiellement en tresses dans une large plaine alluviale formait des bras secondaires (lônes) offrant une vaste mosaïque d'habitats aquatiques et de milieux humides très diversifiés. Dans la seconde moitié du $19^{\mathrm{e}}$ siècle, la construction de digues longitudinales accompagnées d'épis transversaux a concentré les eaux dans un chenal principal et des bras secondaires aux berges stabilisées; le Rhône a été compartimenté en « casiers » (Fruget, 2003). À partir de 1950, les aménagements de la CNR ont créé des retenues et des canaux de dérivation, et ainsi rendu le fleuve navigable sur $310 \mathrm{~km}$. 
Entre 1948 et 1986, dix-huit aménagements ont été réalisés sur le Rhône. Quinze d'entre eux sont des aménagements au fil de l'eau conçus selon le même schéma (Fig. 2) : un barrage de dérivation constitue une retenue en amont, l'eau est dérivée dans un canal d'amenée vers une usine hydroélectrique équipée d'une écluse, après turbinage elle est évacuée dans le canal de fuite. En aval du barrage subsiste un tronçon courtcircuité (le vieux Rhône) qui reçoit, en dehors des périodes de crues, un débit réservé variable selon les aménagements (par exemple de $10 \mathrm{~m}^{3} / \mathrm{s}$ à $150 \mathrm{~m}^{3} / \mathrm{s}$ à Brégnier-Cordon) et parfois modulé selon la saison.

À Bugey, le Haut-Rhône est considéré comme semi-naturel, le courant reste fort sur toute la largeur du lit. A l'amont du site, la présence de lônes et d'îles délimitant des bras accentue la variété des faciès d'écoulement. Le régime hydraulique est toutefois sous la dépendance du fonctionnement hydraulique des aménagements situés en amont.

À l'aval de Lyon, les CNPE sont installés sur des retenues (Saint-Alban/ Saint-Maurice-l'Exil sur la retenue de Péage-de-Roussillon et Cruas-Meysse

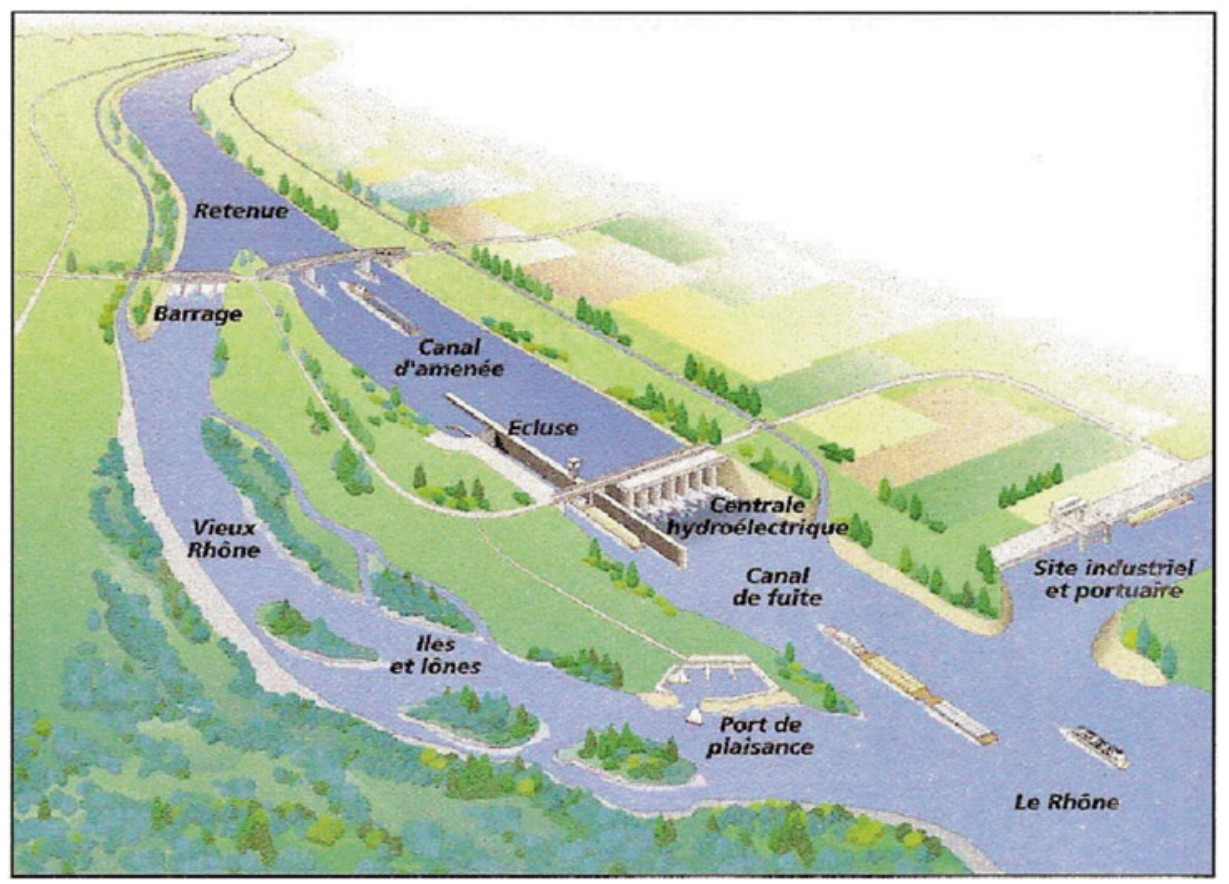

Fig. 2. Schéma de l'aménagement type du Rhône (six aménagements sur le Haut-Rhône et 12 sur le Bas-Rhône). Document CNR.

Fig. 2. Diagram of the standard hydraulic scheme on the Rhône river (six hydro plants on the Upper Rhône and 12 on the Lower Rhône). CNR document. 
sur la retenue de Montélimar) ou sur le canal de dérivation (Tricastin sur le canal de Donzère-Mondragon).

La vallée est ainsi cloisonnée longitudinalement par une succession de barrages, de seuils sur les tronçons court-circuités. Les échanges latéraux sont souvent supprimés, l'enfoncement du lit conduit à abaisser le niveau de la nappe alluviale (Fruget, 2003).

En regard de ces modifications hydro-morphologiques majeures et extensives de l'espace fluvial rhodanien, les seuils, les dispositifs de prise d'eau et de rejet créés pour les besoins des CNPE ont un impact physique réduit par leur faible emprise spatiale.

En aval de Lyon, sur le chenal principal du Rhône, en dehors des tronçons court-circuités, toutes les masses d'eau délimitées au titre de la DCE ont été pré-identifiées dans la catégorie des masses d'eau fortement modifiées (MEFM) en raison des impacts hydro-morphologiques identifiés dans l'état des lieux dressé par l'agence de l'eau RM\&C (Agence de
l'Eau Rhône Méditerranée \& Corse, 2004, 2005). En revanche, la masse d'eau du secteur non canalisé de Bugey (entre Sault-Brénaz et le pont de Jons), n'a pas été classée fortement modifiée; les impacts hydromorphologiques y ont été considérés comme faibles (Tab. II).

Bien que le régime hydrologique du Rhône soit marqué par sa relative régularité en raison de la multiplicité de ces apports (glaciaires, nivo-glaciaires, nivaux et pluviaux), au cours de la période d'étude il a été marqué par une grande variabilité de l'hydraulicité annuelle, avec des années sèches telles que 1989, 2003 et 2004 et des crues exceptionnelles (Fig. 3) dont les effets biologiques ont été sensibles sur le Bas-Rhône. Par ailleurs, la régulation du débit en sortie du Léman, les apports des affluents et la gestion de l'eau à court terme dans les retenues fonctionnant partiellement par éclusées ont modifié le régime hydrologique du fleuve.

Sur la section non aménagée de Bugey, des fluctuations de débit sont

Tableau II. Impacts hydro-morphologiques décrits dans l'état des lieux du Bassin RMC (Agence de l'Eau Rhône Méditerranée \& Corse, 2004). MEN: masse d'eau naturelle. MEFM: Masse d'eau fortement modifiée. PMRH: Prélèvements et modifications du régime hydrologique. OT: Ouvrages transversaux affectant la continuité amont-aval. Amgt: Aménagements (fonctionnement des milieux connexes)

Table II. Hydro-morphological impact described, in WFD characteristics, document regarding the RMC hydrographic district. MEN: natural water body. MEFM: heavily modified water body. PMRH: Water abstraction and modification of water regime. OT: Cross section obstacles limiting upstreamdownstream continuity. Amgt: equipment limiting lateral connectivity.

\begin{tabular}{lccccc}
\hline Limites amont-aval & Type de masse d'eau & CNPE/CPT & PMRH & OT & Amgt \\
\hline Sault-Brenaz - Jons & ME & Bugey & Faible & Faible & Faible \\
Jons - Saône & MEFM & & Moyen & Fort & Fort \\
Saône - Isère & MEFM & Saint-Alban & Moyen & Fort & Fort \\
Isère - Avignon & MEFM & Cruas-Tricastin & Faible & Moyen & Fort \\
Avignon - Beaucaire & MEFM & Aramon & Faible & Moyen & Fort \\
\hline
\end{tabular}




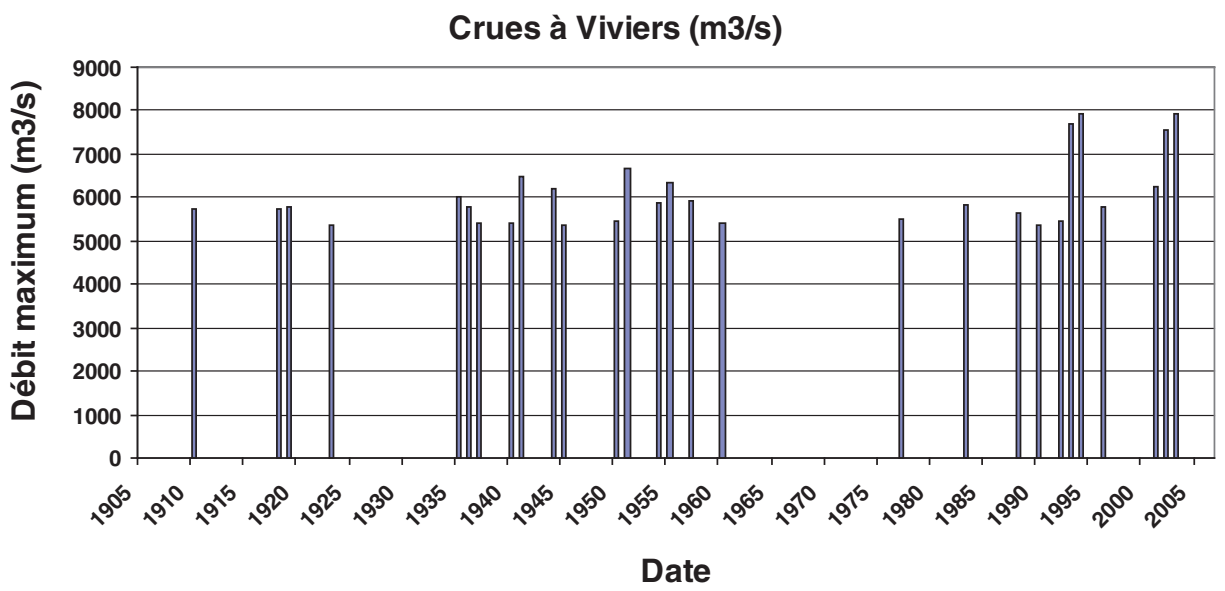

Fig. 3. Les grandes crues du Rhône depuis le début du XXe siècle (CNR, 2004).

Fig. 3. The main flooding events of the Rhone river since the beginning of the 20th century (CNR, 2004).

observées à court terme, allant de quelques heures à quelques jours, sous l'effet de variations imposées à la frontière suisse et/ou du régime d'éclusées dans les retenues gérées par la CNR. La fréquence accrue des très bas débits, voisins de $200 \mathrm{~m}^{3} / \mathrm{s}$, pourraient avoir des effets négatifs sur les stocks de poissons en « tirant vers le bas " le volume d'habitat disponible pour toute la faune aquatique (Souchon et al., 2005).

Dans la perspective du plan de gestion du district établi pour atteindre les objectifs de la DCE, il est envisagé de «modifier la gestion des ouvrages existants afin de tirer bénéfice de leur disponibilité en eau tout en réduisant les impacts sur les milieux aquatiques » (Comité de bassin Rhône Méditerranée \& Corse, 2004). L'augmentation des débits réservés dans les tronçons court-circuités, dont la valeur écologique est reconnue et soulignée dans l'état des lieux du bassin, constitue un des éléments de la restauration de ces milieux. Cette politique a été adoptée dans le cadre du programme décennal de restauration écologique et hydraulique du fleuve (« Programme décennal de restauration hydraulique et écologique du fleuve Rhône », DIREN Délégation de bassin RMC - 3 janvier 2000) et dans le nouveau plan Rhône approuvé en CIACT (Comité interministériel d'aménagement et de compétitivité des territoires) du 6 mars 2006.

\subsection{Le régime thermique}

Grâce aux mesures en continu de la température de l'eau effectuées sur le bassin rhodanien, notamment les mesures intégrées sur une heure à l'amont et à l'aval des centrales thermiques exploitées par EDF, le régime du Rhône a pu être caractérisé à diverses échelles spatio-temporelles (Poirel et al., 2008). Ainsi, la variabilité thermique à court et moyen terme a pu 
être identifiée au même titre que l'évolution thermique sur le long terme par des métriques adaptées en fonction des cycles biologiques des espèces considérées.

\subsubsection{Variations spatiales de température}

\section{Évolution longitudinale} de la température du Rhône

Sur l'axe principal du fleuve, le régime thermique du Rhône est principalement déterminé par la température de l'eau sortant du lac Léman et par celle des principaux affluents. Ces affluents produisent en été une discontinuité thermique plus ou moins importante en refroidissant le fleuve (Arve, Ain et surtout Isère) ou en le réchauffant (Saône) (Fig. 4).

\section{Hétérogénéité spatiale}

Lorsque le fleuve est considéré sur son axe latéral, et notamment à l'échelle d'un aménagement, des différences thermiques importantes existent entre le chenal principal (canaux, retenues, chenal unique) et les annexes (Vieux Rhône et ses anciens bras). Les variations de températures liées aux apports souterrains (nappe alluviale) peuvent être sensibles mais les échanges atmosphériques deviennent localement prépondérants. Les apports de nappe, plus froids en été que l'eau du fleuve, créent une mosaïque thermique près $\mathrm{du}$ fond. Les zones les plus froides peuvent abriter momentanément des organismes et leur permettre de passer sans dommage les périodes critiques de maximum thermique.

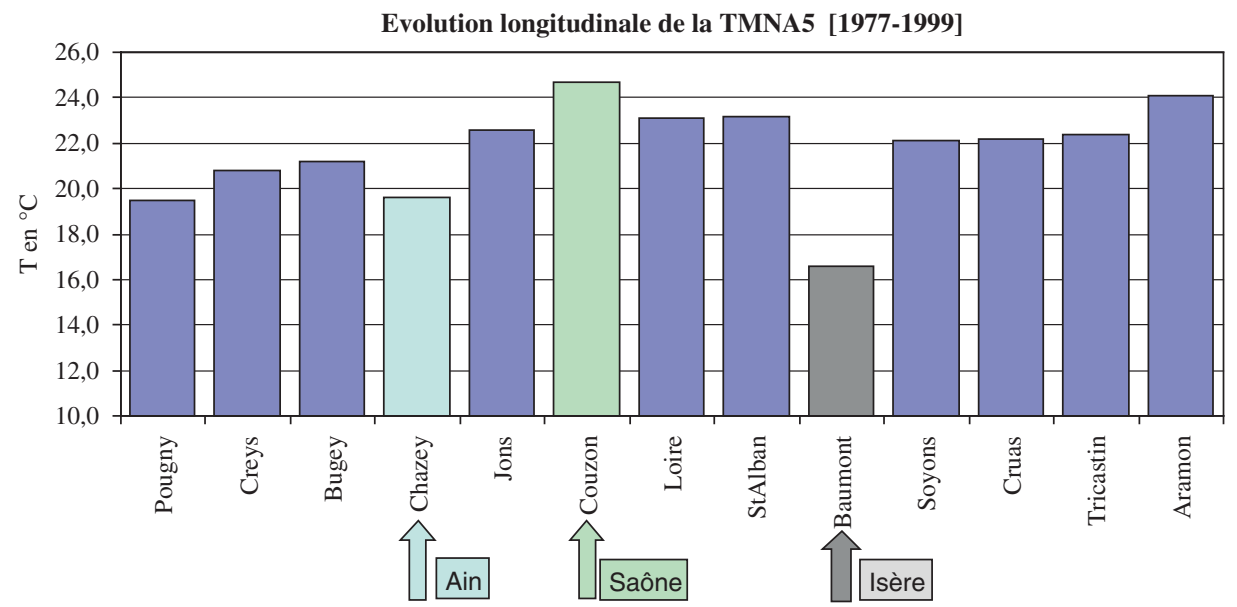

Fig. 4. Évolution longitudinale de la température du mois le plus chaud de période de retour 5 ans (TMNA5). Cette variable met en évidence les contrastes thermiques entre le Rhône et ses principaux affluents (Ain, Saône, Isère) et leur influence respective. Période 1977 à 1999 (Poirel et al., 2001).

Fig. 4. Longitudinal evolution of the warmest month temperature with a 5-year return period (TMNA5). This variable points out the thermal contrast between the Rhone river and its main tributaries (Ain, Saône, Isère) and their respective influence. 1977-1999 time series (Poirel et al., 2001). 
Ces « refuges thermiques » peuvent également fonctionner l'hiver avec des apports de nappe plus chauds que le cours d'eau. Lorsque les affluents ne présentent pas de discontinuités avec le chenal principal, en absence d'ouvrages constituant des obstacles, ils peuvent également représenter des zones refuges pour les poissons.

\subsubsection{Changement à long terme}

Au cours des 25 dernières années, le changement le plus remarquable est une augmentation très significative de la température moyenne annuelle (Fig. 5, Tab. III; Desaint, 2004). Une tendance similaire est observée pour les affluents du Rhône (Carrel, 2006b) et pour la Loire (Moatar \& Gailhard, 2006).
Sur la période considérée (19772004), cette augmentation de la température de l'eau est plus marquée dans le Bas-Rhône qu'en amont de Lyon. À l'amont immédiat des sites de production, l'écart sur la température moyenne annuelle varie entre $+1,6^{\circ} \mathrm{C}$ à Bugey et $+3,0^{\circ} \mathrm{C}$ à Aramon. La chronique, marquée par des années froides en début de période et par l'année record 2003 en fin de période, exacerbe cette tendance.

Les écarts maximum sur les moyesnnes annuelles atteignent $2,8^{\circ} \mathrm{C}$ à Bugey et $4,2^{\circ} \mathrm{C}$ à Aramon (Fig. 5), (Carrel, 2006a).

Le régime thermique du fleuve a été analysé (Carrel, 2006a) en découpant l'année en 5 périodes successives sur la base de seuils thermiques ayant une signification

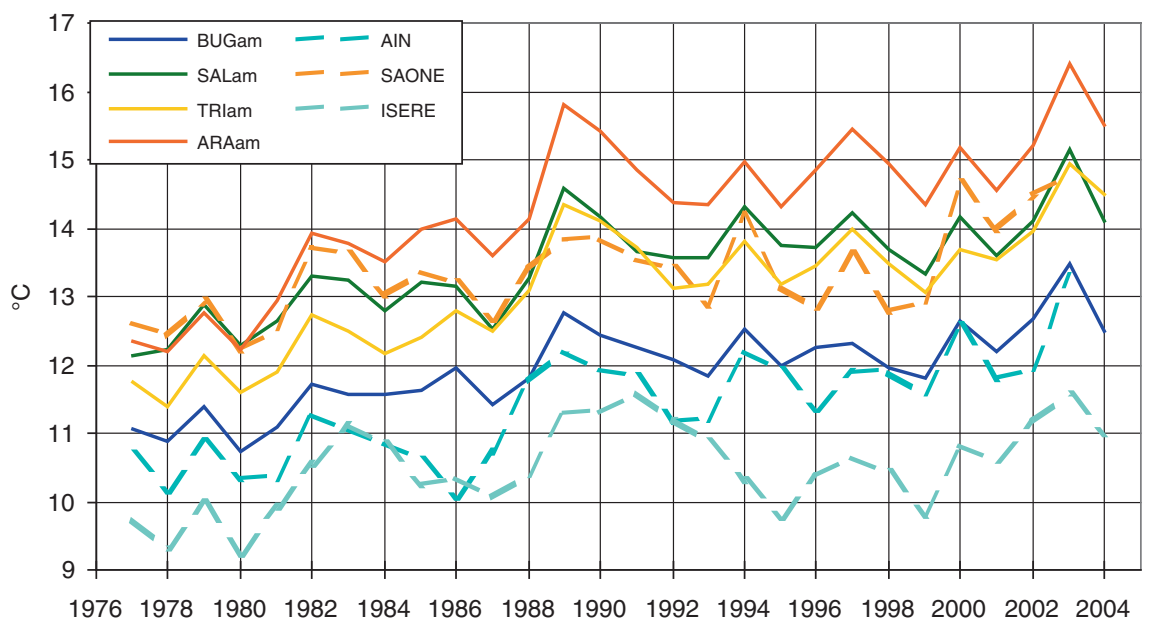

Fig. 5. Évolution de la température moyenne annuelle entre 1977 et 2004 sur les quatre stations du Rhône situées en amont des CNPE, et les trois principaux affluents : Ain, Saône et Isère.

Fig. 5. Evolution of the mean annual temperature between 1977 and 2004 at 4 Rhone river stations (upstream of NPP) and 3 main tributaries: Ain, Saône and Isère. 
Tableau III. Évolution de la température moyenne sur des périodes de cinq ans entre 1977 et 2004 sur les quatre stations du Rhône situées en amont des CNPE, et les trois principaux affluents : Ain, Saône et Isère.

Table III. Evolution of the five-year mean annual temperature between 1977 and 2004 at 4 Rhone river stations (upstream of NPP) and 3 main tributaries: Ain, Saône and Isère.

\begin{tabular}{lcccccc}
\hline Période & $1977-79$ & $1980-84$ & $1985-89$ & $1990-94$ & $1995-99$ & $2000-04$ \\
\hline BUGam & 11,1 & 11,3 & 11,9 & 12,2 & 12,1 & 12,7 \\
SALam & 12,4 & 12,8 & 13,3 & 13,9 & 13,7 & 14,2 \\
TRlam & 11,8 & 12,2 & 13,0 & 13,6 & 13,4 & 14,1 \\
ARAam & 12,4 & 13,3 & 14,3 & 14,8 & 14,8 & 15,4 \\
Ain & 10,6 & 10,8 & 11,1 & 11,7 & 11,7 & 12,4 \\
Saône & 12,7 & 13,0 & 13,3 & 13,6 & 13,1 & 14,5 \\
Isère & 9,7 & 10,3 & 10,5 & 11,1 & 10,2 & 11,0 \\
\hline
\end{tabular}

* sans 2004

biologique ${ }^{(1)}$. Le découpage est réalisé à partir d'un lissage annuel par séries de Fourier de la courbe des températures journalières. Dans l'exemple donné (Fig. 6), les périodes répondent aux définitions suivantes :

- du minimum thermique annuel de l'année (n) au seuil printanier $12^{\circ} \mathrm{C}$,

- du seuil printanier $12^{\circ} \mathrm{C}$ au seuil $18^{\circ} \mathrm{C}$,

- du seuil estival $18^{\circ} \mathrm{C}$ au maximum thermique,

- du maximum thermique au seuil automnal $12^{\circ} \mathrm{C}$,

- du seuil automnal $12^{\circ} \mathrm{C}$ au minimum thermique de l'année $(n+1)$.

Pour chaque période, trois variables ont été créées (Fig. 6) :

(1) Le choix fait dans cette présentation concerne principalement la reproduction des cyprinidés et le lecteur pourrait être surpris par les seuils fixés. La méthode utilisée peut être adaptée en regard des préoccupations des biologistes et des organismes étudiés.
- C : le jour de l'année fixant le début de la période compté à partir du $1^{\mathrm{er}}$ janvier,

- D : la durée de chaque période en jours,

- S : la somme des écarts thermiques pour chaque période calculée par différence entre les courbes lissées inter-annuelle et annuelle, et équivalente à un cumul de degrés-jours référencés par la courbe lissée inter-annuelle.

L'analyse des données a montré quatre tendances caractérisant le changement climatique des dernières décennies.

(1) Une augmentation de la somme des écarts thermiques $S$ a été observée sur les 5 périodes, avec un maximum pour les variables S3 et S4 correspondant respectivement à la somme des écarts thermiques entre le seuil $18^{\circ} \mathrm{C}$ et le maximum annuel, et à la somme des écarts thermiques entre le maximum et le seuil automnal $12^{\circ} \mathrm{C}$.

(2) Les seuils thermiques $12^{\circ} \mathrm{C}$ et surtout $18^{\circ} \mathrm{C}$ sont de plus en plus précoces. 
Température $\left({ }^{\circ} \mathrm{C}\right)$

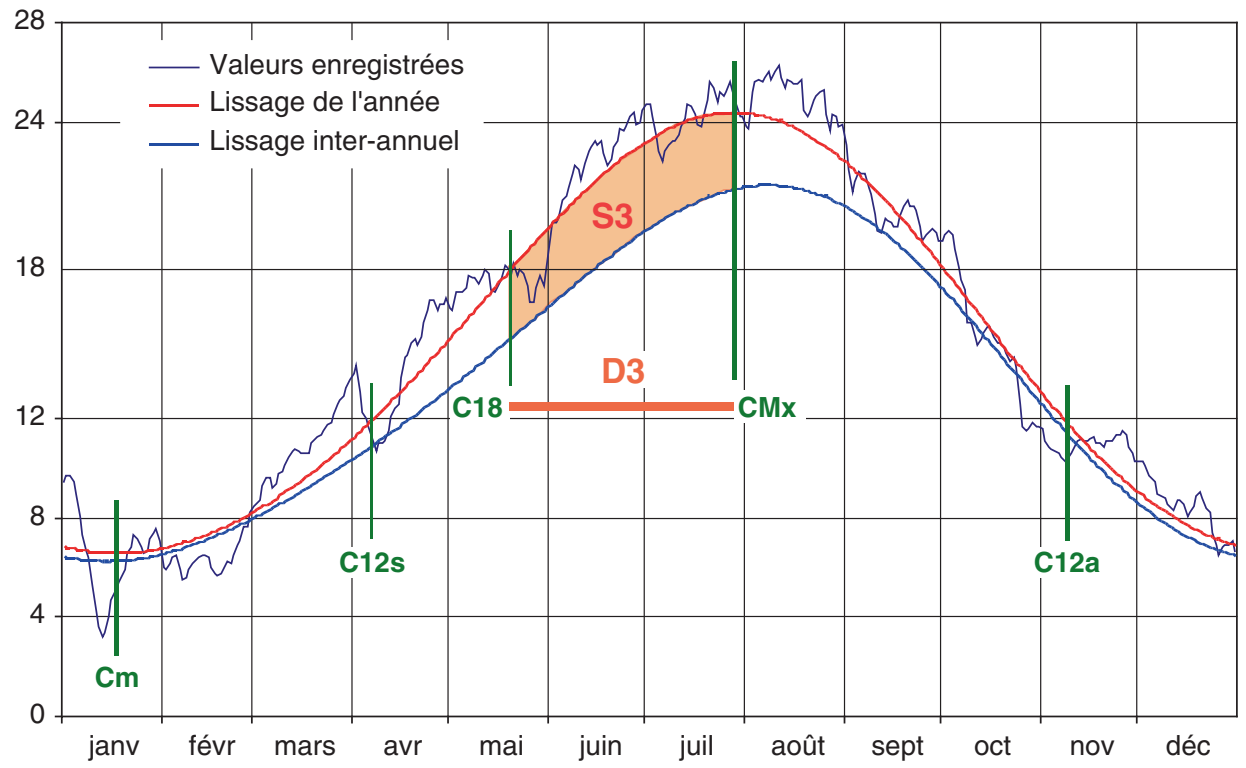

Fig. 6. Variables thermiques à Tricastin amont pour l'année 2003. La courbe supérieure (rouge) est la température lissée autour des valeurs moyennes journalières, la courbe inférieure (bleue) est obtenue par lissage sur la série complète de valeurs (1977 à 2004). Les valeurs des variables indiquées (C, S \& D) se rapportent à l'année 2003. Par exemple, S3 (somme en degrés-jours) est l'aire comprise entre les deux courbes, limitée par $\mathrm{C} 18$ (seuil $18^{\circ} \mathrm{C}$ ) et par $\mathrm{CMx}$ (maximum thermique annuel).

Fig. 6. Temperature upstream of Tricastin during the year 2003. The upper line (red) represents the 2003 smoothed average daily temperature values, the lower line (blue) the smoothed values over the 1977-2004 period. Values of variables (C, S \& D) are presented for the year 2003. (Cm: date of the minimum temperature; $\mathrm{C} 12$ and $\mathrm{C} 18$ beginning of the period above the 12 or $18^{\circ} \mathrm{C}$ threshold; $\mathrm{CMx}$ : date of the maximum temperature), for example S3 (sum in degree-days) is the area between the two smoothed curves, from $\mathrm{C} 18$ to $\mathrm{CMx}$.

(3) La durée des périodes D1 ( $\mathrm{Cm}$ à $\mathrm{C} 12 \mathrm{~s})$ et $\mathrm{D} 2$ (C12s à C18) a diminué et celle des périodes D3 et D4 s'est allongée.

(4) La période D5, la plus froide, s'est raccourcie.

\subsubsection{Les variations thermiques à moyen terme (de la semaine au mois)}

Les fluctuations conjuguées des débits respectifs du Rhône et de ses affluents, et des facteurs météoro- logiques induisent des variations de température sensibles à l'échelle de la semaine et du mois. Une des caractéristiques remarquables du régime thermique du Rhône réside dans les épisodes de chute brutale de la température qui durent de quelques jours à plusieurs semaines. Ils sont provoqués par la remontée d'eau profonde et froide de l'hypolimnion lémanique. Qualifiés de «gouttes froides », ils sont particulièrement visibles en saison chaude (Fig. 7). 


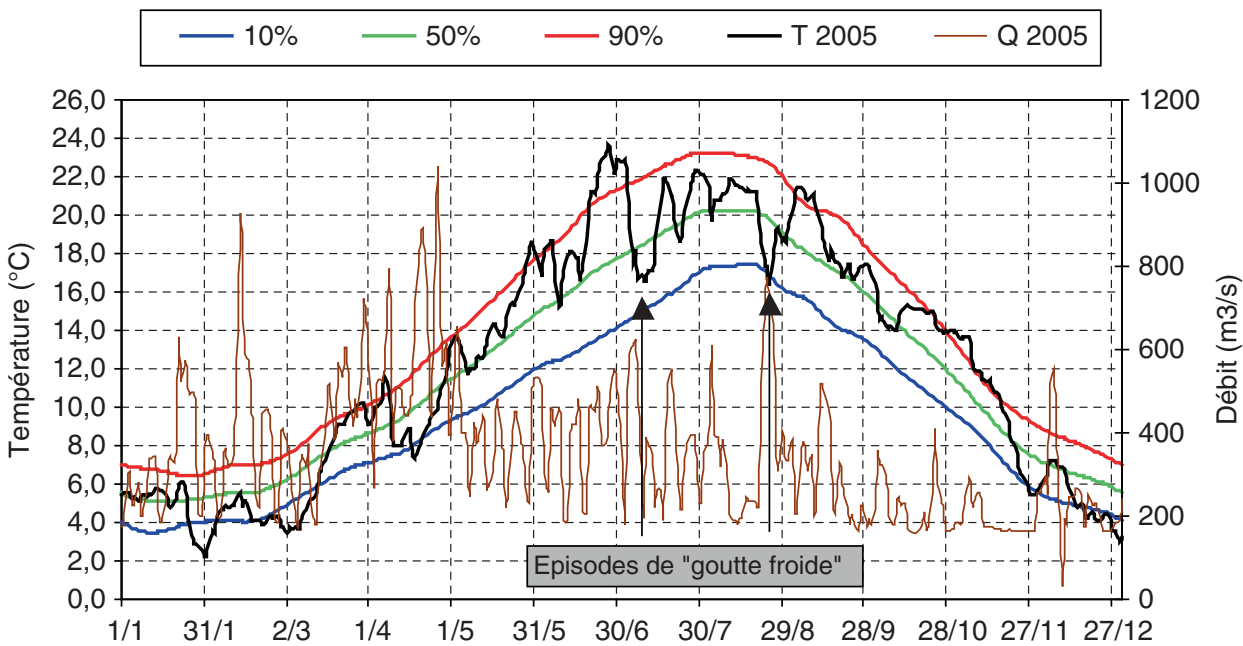

Fig. 7. Illustration des chroniques thermique et hydrologique du Rhône en amont du CNPE de Bugey en 2005 et localisation d'une succession d'épisodes qualifiés de " gouttes froides " marqués par une chute brutale de la température estivale. Évolution des quantiles de température (10\%, 50 \%, $90 \%)$ sur la période 1977-2004 (Daufresne \& Boët, 2005).

Fig. 7. Time series of mean daily temperatures and water discharges in 2005 upstream of Bugey NPP. Evolution of temperature percentiles (10\%, 50\%, 90\%) over the 1977-2004 period and brutal decrease of water temperature (gouttes froides) caused by cold water output from the deep layer of the Geneva Lake (Daufresne \& Boët, 2005).

Sur le Haut-Rhône, la chute de température peut atteindre $9^{\circ} \mathrm{C}$ en 24 heures. Les gouttes froides balaient ensuite le Rhône en se déplaçant vers l'aval avec une vitesse de propagation nettement plus lente que la propagation des variations de débit. Sur les données relatives à la goutte froide de fin juin 2003 représentées sur la figure 8 , deux traits majeurs de sa propagation apparaissent clairement, l'atténuation progressive de l'amplitude thermique et une augmentation de la durée de l'épisode vers l'aval.

\subsubsection{Les variations thermiques à court terme (de l'heure à la journée)}

De par leurs conditions morphodynamiques spécifiques, les tronçons du Rhône court-circuité (RCC) et les annexes hydrauliques peu profondes et à faible écoulement présentent en période estivale un régime thermique caractérisé par des variations nycthémérales de plus vaste amplitude que dans le chenal principal. Ces phénomènes sont renforcés par la présence de seuils dans les tronçons courtcircuités.

Dans le chenal principal du Rhône, les variations nycthémérales restent de faible amplitude. Sur la période 1977-1999, l'amplitude moyenne a été de $0,7^{\circ} \mathrm{C}$ à Bugey et de $0,6^{\circ} \mathrm{C}$ à l'amont de Saint-Alban dans la retenue. Pendant les mois de juin à septembre, l'amplitude moyenne a été de $1,1^{\circ} \mathrm{C}$ à Bugey et seulement de $0,7^{\circ} \mathrm{C}$ à l'amont de Saint-Alban. 
Transfert de la "goutte froide" de la fin du mois de juin 2003

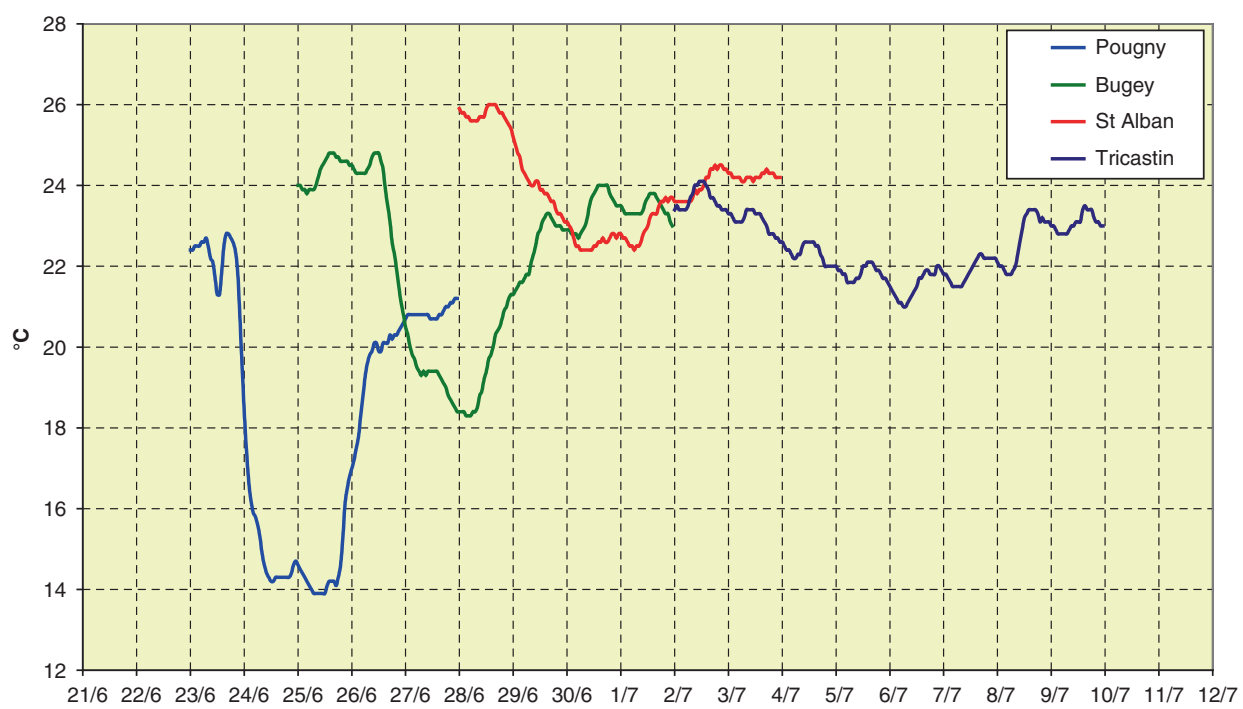

Fig. 8. Évolution de la température du Rhône depuis Pougny jusqu'à Tricastin lors d'un épisode de goutte froide en juin-juillet 2003 (Desaint, 2004).

Fig. 8. Evolution of the Rhône river temperature between Pougny and Tricastin during a Geneva Lake cold water output event in June-July 2003 (Desaint, 2004).

Dans les tronçons court-circuités, l'étalement de la lame d'eau sur un lit large et peu profond favorisent les échanges thermiques air-eau. Les variations nycthémérales peuvent alors dépasser $4^{\circ} \mathrm{C}$. En 2003, l'amplitude journalière dans le tronçon courtcircuité de l'aménagement de Péagede-Roussillon en aval de Saint-Alban a atteint $2,7^{\circ} \mathrm{C}$ en moyenne avec un maximum de $5,0^{\circ} \mathrm{C}$. Par comparaison, l'amplitude journalière à l'entrée du canal de dérivation, à la station « $S A L$ aval ", était de $1,0^{\circ} \mathrm{C}$ en moyenne et de $2,5^{\circ} \mathrm{C}$ au maximum (Tab. IV, Fig. 9).

À Péage-de-Roussillon, seule section du Rhône où un rejet thermique est susceptible de modifier le régime thermique d'un ancien chenal du fleuve par un apport direct, la température des eaux de l'ancien Rhône est essentiellement dépendante des conditions climatiques externes. Malgré le contexte hydraulique local, la température est nettement moins influencée par l'échauffement résiduel du CNPE de Saint-Alban que ne le sont le chenal, les canaux d'amenée et de fuite de l'usine hydro-électrique.

\subsubsection{Modifications induites par les rejets des CNPE}

Afin de comparer les échauffements résiduels résultant des rejets thermiques, d'une part et la dérive climatique, d'autre part, des données 
Tableau IV. Températures moyennes et amplitudes thermiques dans le Rhône et dans le tronçon court-circuité (RCC) de Péage-de-Roussillon en aval du CNPE de Saint-Alban en 2003.

Table IV. Average temperature and thermal range in the Rhône river and in the by-passed section (RCC) of Péage-de-Roussillon, downstream of Saint-Alban NPP, in 2003.

\begin{tabular}{|c|c|c|c|c|c|}
\hline \multirow[t]{2}{*}{ Station } & \multicolumn{2}{|c|}{ Température moyenne } & \multicolumn{2}{|c|}{ Amplitude journalière } & \multirow{2}{*}{$\begin{array}{c}\text { Amplitude } \\
\text { maximale } \\
\text { journalière }\end{array}$} \\
\hline & annuelle & $\begin{array}{c}\text { de juin à } \\
\text { septembre }\end{array}$ & $\begin{array}{l}\text { moyenne } \\
\text { annuelle }\end{array}$ & $\begin{array}{c}\text { de juin à } \\
\text { septembre }\end{array}$ & \\
\hline Saint-Alban aval* & $16,5^{\circ} \mathrm{C}$ & $25,1^{\circ} \mathrm{C}$ & $1,0^{\circ} \mathrm{C}$ & $1,3^{\circ} \mathrm{C}$ & $2,5^{\circ} \mathrm{C}$ \\
\hline $\mathrm{RCC}$ & $16,5^{\circ} \mathrm{C}$ & $23,8^{\circ} \mathrm{C}$ & $2,7^{\circ} \mathrm{C}$ & $3,4^{\circ} \mathrm{C}$ & $5,0^{\circ} \mathrm{C}$ \\
\hline
\end{tabular}

*station mi-rejet (voir carte du secteur en annexe).

Amplitudes journalières à l'aval de St Alban pendant l'été 2003

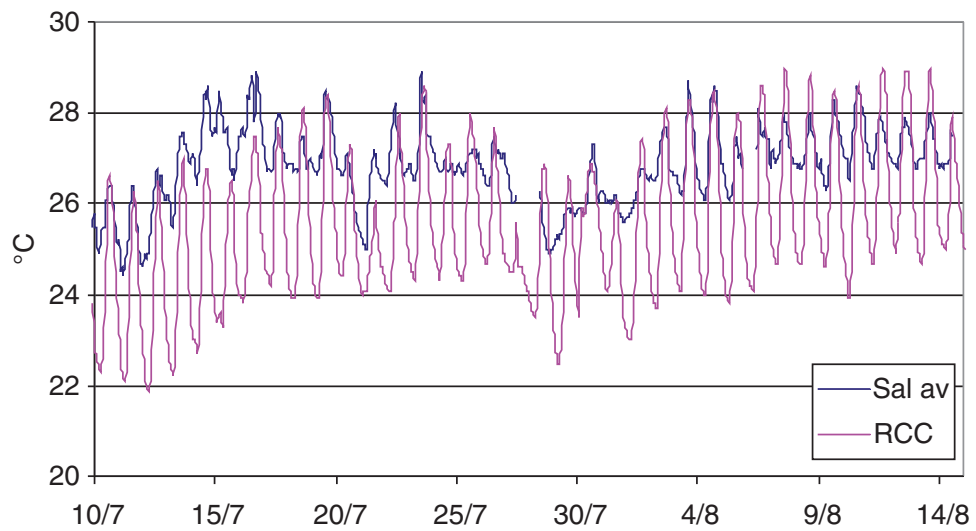

Fig. 9. Chronique des températures mesurées à la station aval de Saint-Alban (Sal mi-rejet) et dans le Rhône court-circuité (RCC) en juillet et août 2003. La position des stations est donnée sur la carte en annexe.

Fig. 9. Time series of temperature measured at the monitoring station (Sal mi-rejet) downstream of Saint-Alban and in the by-passed section (RCC) in July and August 2003. The sampling sites location is presented on the map in appendix.

de l'étude thermique phase 2 (Desaint, 2004) ont été synthétisées sur la figure 10. Le site de Cruas dont toutes les tranches sont en circuit fermé n'a pas été pris en compte en raison d'apports thermiques négligeables. En un point donné, l'échauffement résiduel est évalué par calcul à partir des rejets thermiques des CNPE situés en amont : ainsi, au niveau d'Aramon, le calcul de l'échauffement résiduel tient compte des rejets de Bugey, SaintAlban et Tricastin.

L'échauffement résiduel moyen à l'amont des CNPE du Bas-Rhône $\left(+0,6^{\circ} \mathrm{C}\right.$ à $\left.+1,4^{\circ} \mathrm{C}\right)$ est nettement 


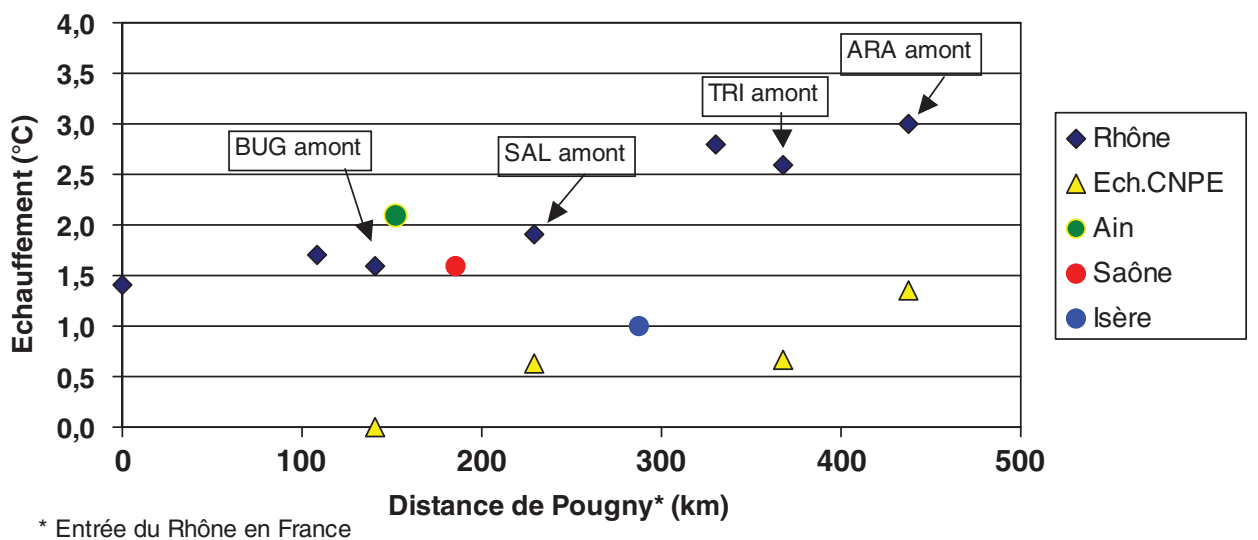

Fig. 10. Échauffements résiduels moyens liés aux rejets des CNPE de Bugey, Saint-Alban, Tricastin et le CPT d'Aramon (période 1991-2003, triangles jaune) et élévation de la température moyenne annuelle entre 1977 et 2004 sur 7 stations du Rhône de Pougny à Aramon et sur les principaux affluents.

Fig. 10. Residual temperature rise resulting from Bugey, Saint-Alban, Tricastin NPP discharges (1991-2003 mean, yellow triangles) and elevation of mean annual temperature between 1977 and 2004 at 7 stations on the Rhône river between Pougny (Swiss border) and Aramon and on the main tributaries.

inférieur à l'élévation de température moyenne constatée entre 1977 et 2004 qui varie de $+1,5^{\circ} \mathrm{C}$ à l'amont du CNPE de Bugey à $+3,0^{\circ} \mathrm{C}$ en amont du centre de production thermique d'Aramon.

Pour éviter une interprétation abusive de ces courbes, il faut attirer l'attention sur deux points :

- d'une part, il s'agit d'échauffements résiduels moyens causés par les rejets des CNPE, ce qui implique que des valeurs plus élevées sont observées,

- d'autre part, l'effet climatique évalué d'après la tendance linéaire des températures sur la période 1977-2004, ne se cumule pas directement aux échauffements des rejets thermiques; en effet, les échauffements observés ne sont pas rigoureusement égaux à la somme des échauffements dus aux facteurs climatiques seuls et de ceux induits par les rejets des CNPE.

Quoiqu'il en soit, dans l'hypothèse d'une poursuite de la tendance climatique sans modification significative de l'hydrologie, la part des apports thermiques par les centrales devrait diminuer en relatif dans l'avenir.

\subsection{La qualité de l'eau}

\subsubsection{Paramètres classiques}

Une étude des paramètres relevés sur les 13 stations du bassin rhodanien faisant l'objet d'un suivi permanent (Carrel, 2006b), met en évidence les différences existantes entre le 
Haut-Rhône (et son affluent l'Ain) d'une part et le Bas-Rhône en aval de Lyon (et la Saône) d'autre part. Bien que les grands tronçons du fleuve présentent une pollution urbaine décroissante de l'amont vers l'aval, la Saône et le Bas-Rhône sont nettement plus minéralisés, plus riches en nitrates et en phosphates. L'Ain présente les caractéristiques du Haut-Rhône, mais son fonctionnement hydraulique se traduit par des épisodes marqués de sursaturation en oxygène dissous.

L'analyse de la variabilité interannuelle indique une baisse du phosphate, de l'azote ammoniacal et de la $\mathrm{DBO}_{5}$, indicateurs de pollutions d'origine urbaine. En revanche, les teneurs en nitrates, principalement d'origine agricole, augmentent. Par ailleurs, la conductivité est en hausse sur le HautRhône parallèlement à la hausse des chlorures dont l'usage hivernal intensif sur le réseau routier est bien perceptible sur le long terme.

Selon la Circulaire DCE 20512 (Combe et al., 2001), les limites de bonne qualité pour l'oxygène dissous se situent dans l'intervalle de 6 à $8 \mathrm{mg} / \mathrm{L}$ au centile 90. Sur tout le Rhône, l'oxygénation de l'eau reste satisfaisante, même lors de l'épisode caniculaire de 2003 (Tab. V). À cette occasion, il a été observé des variations nycthémérales très importantes d'oxygène dissous dans le tronçon court-circuité à Péage-de-Roussillon ; la végétation fixée est responsable de ces variations dans ce tronçon où l'eau s'écoule sur une faible profondeur.

L'état des lieux réalisé au titre de l'application de la DCE (Ministère de l'écologie et du développement durable, 2005), prévoit l'atteinte du bon état physico-chimique en 2015 (Tab. VI).

\subsubsection{Polluants potentiellement toxiques}

Malgré une réduction des rejets de substances potentiellement toxiques sur les sites industriels du " corridor de la chimie " en aval de Lyon, l'état des lieux de la DCE considère que la qualité chimique restera moyenne pour les métaux et sur une partie du fleuve pour les pesticides et les micropolluants organiques. Le

Tableau V. Concentration en oxygène dissous (mg/L) de juin à septembre 2003.

Table V. Dissolved oxygen concentration (mg/L) from June to September 2003.

\begin{tabular}{lccc}
\hline CNPE & Sites & Percentile 25 & Percentile 75 \\
\hline Bugey & Amont CNPE & 7,0 & 8,1 \\
& Aval CNPE & 7,1 & 8,5 \\
Saint-Alban & Amont CNPE & 6,6 & 7,7 \\
& Aval CNPE & 6,5 & 7,9 \\
Rhône & RCC péage & 4,6 & 8,6 \\
Cruas & Amont CNPE & 6,0 & 7,2 \\
& Aval CNPE & 6,3 & 7,8 \\
Tricastin & Amont CNPE & 6,3 & 8,4 \\
& Aval CNPE & 5,9 & 7,1 \\
\hline
\end{tabular}


Tableau VI. Qualité physico-chimique prévue en 2015 d'après l'état des lieux du Bassin RM\&C (Agence de l'Eau Rhône Méditerranée \& Corse, 2004). MOOX : matières organiques oxydables, AZOT : matières azotées, NITR : nitrates, PHOS : matières phosphorées. Bon : bon état.

Table VI. Physico-chemical quality predicted in 2015 based on the WFD characteristics document for the RMC hydrographic district (Agence de l'Eau Rhône Méditerranée \& Corse, 2004). MOOX: oxidable organic matter, AZOT: nitrogen-containing compounds, NITR: nitrates, PHOS: phosphorus-containing compounds, Bon: good status.

\begin{tabular}{lcccccc}
\hline Limites amont-aval & Type de masse d'eau & CNPE/CPT & MOOX & AZOT & NITR & PHOS \\
\hline Sault-Brenaz - Jons & MEN & Bugey & Bon & Bon & Bon & Bon \\
Jons - Saône & MEFM & & Bon & Bon & Bon & Bon \\
Saône - Isère & MEFM & Saint-Alban & Bon & Bon & Bon & Bon \\
Isère - Avignon & MEFM & Cruas-Tricastin & Bon & Bon & Bon & Bon \\
Avignon - Beaucaire & MEFM & Aramon & Bon & Bon & Bon & Bon \\
\hline
\end{tabular}

compartiment sédimentaire constitue un réservoir de polluants susceptibles de contaminer les organismes aquatiques vivant dans les sédiments ou par remise en suspension lors des crues.

À l'occasion de campagnes de mesures réalisées en 2005 et 2006, la présence de polychlorobiphényles (PCB) a été détectée dans des poissons du Rhône pêchés dans la région lyonnaise à des concentrations supérieures à la limite indiquée dans le règlement CE 199/2006. Des initiatives ont été prises par les autorités en vue de connaître l'état de la contamination et de gérer le risque sanitaire en recourant à des interdictions de consommation de poissons. Les analyses réalisées par le Cemagref sur les sédiments dans lesquels s'accumulent les substances hydrophobes telles que les PCB, indiquent qu'il s'agit d'une pollution étendue, causée par des apports diffus et essentiellement ancienne car les couches profondes des carottes de sédiment sont 5 fois plus contaminées que le sédiment superficiel (Babut et Miege, 2007).

Tableau VII. Qualité chimique prévue en 2015 d'après l'état des lieux du Bassin RMC (Agence de l'Eau Rhône Méditerranée \& Corse, 2004). MTx : métaux, PEST : pesticides, MPOr : micropolluants organiques, Bon : bon état, Moyen : état moyen.

Table VII. Chemical quality predicted in 2015 based on the WFD characteristics document for the RMC hydrographic district (Agence de l'Eau Rhône Méditerranée \& Corse, 2004). MTx: metals, PEST: pesticides, MPOr: organic micropollutants, Bon: good status, Moyen: medium status.

\begin{tabular}{lccccc}
\hline Limites amont-aval & Type de masse d'eau & CNPE/CPT & MTx & PEST & MPOr \\
\hline Sault-Brenaz - Jons & MEN & Bugey & Moyen & Bon & Moyen \\
Jons - Saône & MEFM & & Moyen & Bon & Moyen \\
Saône - Isère & MEFM & Saint-Alban & Moyen & Moyen & Moyen \\
Isère - Avignon & MEFM & Cruas-Tricastin & Moyen & Moyen & Moyen \\
Avignon - Beaucaire & MEFM & Aramon & Moyen & Bon & Bon \\
\hline
\end{tabular}




\section{3 ÉVOLUTION DE LA FAUNE ET DE LA FLORE AQUATIQUE DU RHÔNE EN RELATION AVEC LE RÉGIME THERMIQUE}

\subsection{Macro-invertébrés}

\subsubsection{Structure spatiale et aménagements}

Sur le Haut-Rhône, le peuplement de macro-invertébrés a été affecté par les aménagements (Dessaix et al.,
Tableau VIII. Écart relatif de richesse taxonomique entre l'amont du CNPE de Bugey et l'amont des CNPE de Saint-Alban, Cruas et Tricastin.

Table VIII. Difference in taxonomic richness between sites upstream of Saint-Alban, Cruas et Tricastin NPP and upstream of Bugey NPP.

\begin{tabular}{lcc}
\hline & Totale & Moyenne \\
\hline SAL amont - BUG amont & $-21 \%$ & $-24 \%$ \\
CRU amont - BUG amont & $-34 \%$ & $-24 \%$ \\
TRI amont - BUG amont & $-36 \%$ & $-27 \%$ \\
\hline
\end{tabular}

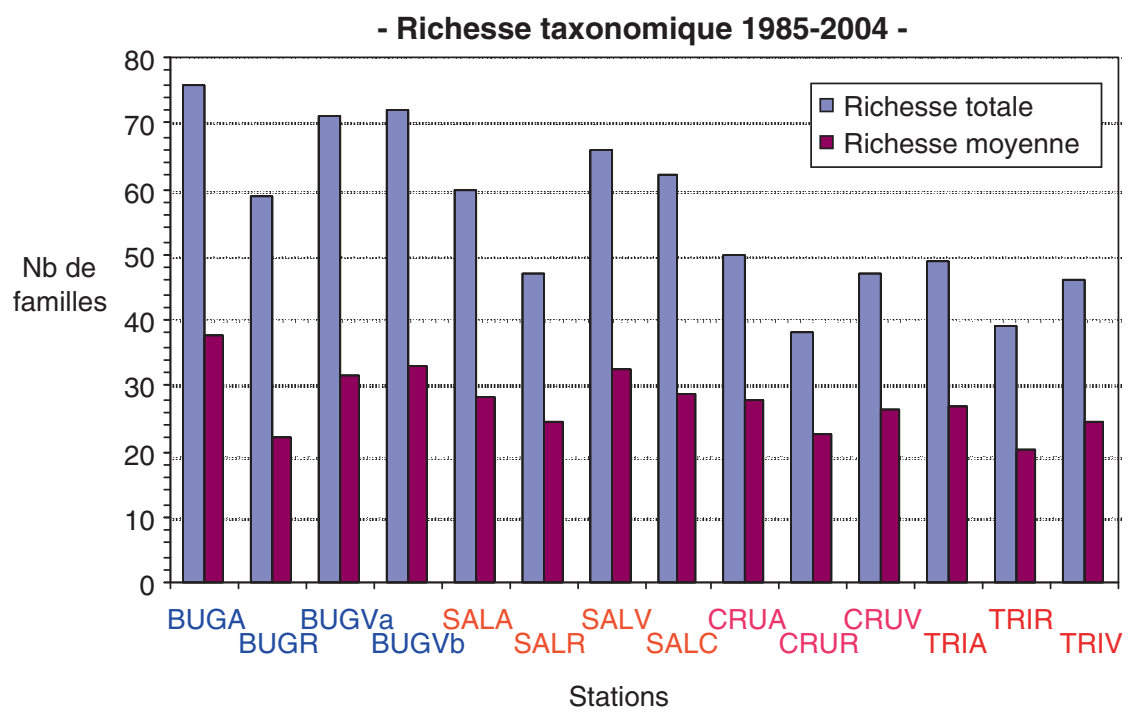

Fig. 11. Richesse taxonomique totale et moyenne annuelle des familles de macro-invertébrés, calculée sur la chronique des données collectées de 1985 à 2004 en amont et en aval des CNPE (Bady \& Fruget, 2006). BUG : Bugey, SAL : Saint-Alban, CRU : Cruas, TRI : Tricastin. A : amont, R : rejet, $\mathrm{V}$ : aval, C : tronçon court-circuité. Localisation des stations sur les cartes en annexe : BUGVa : I3, BUGVb : 14, SALV : 3.

Fig. 11. Total taxonomic richness and yearly average richness of macroinvertebrates families, based on data collected from 1985 to 2004 upstream and downstream of NPP (Bady \& Fruget, 2006). BUG: Bugey, SAL: Saint-Alban, CRU: Cruas, TRI: Tricastin. A: upstream, R: discharge, V: downstream, C: by-passed section. See maps in annex for the location of stations: BUGVa: I3, BUGVb: I4, SALV: 3. 
1995) avec une régression ou disparition de larves d'insectes rhéophiles (des Plécoptères, différentes espèces d'Ephémères Heptageniidae et des Trichoptères filtreurs) et le développement concomitant de taxons à affinité lénitique tels que Potamanthus luteus (Ephémèroptère), Polycentropus flavomaculatus et $\mathrm{Hy}$ droptila sp. (Trichoptères), Asellus aquaticus (Crustacé), plusieurs espèces de Mollusques Gastéropodes et de Planaires.

Dans le Rhône à Lyon, une forte réduction du nombre d'espèces de Trichoptères ( $47 \%$ ) et d'Ephémèroptères $(42 \%)$ a été observée après la mise en service du barrage de Pierre-Bénite en 1966 (Usseglio-Polatera, 1985).

La richesse taxonomique totale (tous les taxons observés pendant les vingt ans de mesures) et la richesse taxonomique moyenne annuelle (Fig. 11) diminuent de l'amont vers l'aval.

Par rapport à l'amont de Bugey, les richesses taxonomiques annuelles moyennes à l'amont de SaintAlban, Cruas et Tricastin sont plus basses d'environ $25 \%$ (Tab. VIII). Cette baisse par rapport au milieu semi-naturel de Bugey s'explique par un appauvrissement des taxons d'insectes sur les stations du BasRhône situées dans des retenues. Malgré une différence d'habitat; la charge polluante organique et en substances potentiellement toxiques contribue également à cet écart.

\subsection{2 Évolutions temporelles}

Évolution de la diversité et de l'abondance des macro-invertébrés

Entre 1975 et 1988, Roger et al. (1990) ont montré une perte de richesse taxonomique sur le site de Bugey sur les stations amont et aval du CNPE. Cet appauvrissement a été principalement causé par une disparition de taxons d'insectes (Tab. IX).

Cependant, une augmentation de la richesse taxonomique a été observée au cours des dernières années sur le site de Bugey (Fig. 12).

À Saint-Alban, une augmentation de la richesse taxonomique s'est

Tableau IX. Richesse taxonomique des macro-invertébrés sur le site de Bugey en 1975 et en 1988 (Roger et al., 1990). PLE : Plécoptères, TRI : Trichoptères, EPH : Ephémèroptères, COL : Coléoptères, DIP : Diptères, SAPLA : Sangsues et Planaires, MOLL : Mollusques,

Table IX. Taxonomic richness of macroinvertebrates in the Bugey area in 1975 and in 1988 (Roger et al., 1990). 1: upstream right bank, 3: downstream right bank, 4: downstream left bank. PLE : Plecoptera, TRI: Trichoptera, EPH: Ephemeroptera, COL: Coleoptera, DIP: Diptera, SAPLA: Leeches and Planaria, MOLL: Mollusks.

\begin{tabular}{lccccccccccc}
\hline Station & Année & PLE & TRI & EPH & COL & DIP & SAPLA & MOLL & Divers & Total & Total insectes \\
\hline 1, amont RD & 1975 & 4 & 27 & 10 & 7 & 6 & 6 & 8 & 5 & 73 & 54 \\
& 1988 & 3 & 16 & 6 & 5 & 7 & 6 & 4 & 5 & 52 & 37 \\
3, aval RD & 1975 & 4 & 27 & 10 & 7 & 6 & 6 & 8 & 5 & 73 & 54 \\
\multirow{3}{*}{ 4, aval RG } & 1988 & 4 & 17 & 5 & 6 & 5 & 6 & 4 & 4 & 51 & 37 \\
& 1975 & 4 & 27 & 10 & 7 & 6 & 6 & 8 & 5 & 73 & 54 \\
& 1988 & 1 & 15 & 9 & 13 & 7 & 6 & 7 & 5 & 63 & 45 \\
\hline
\end{tabular}




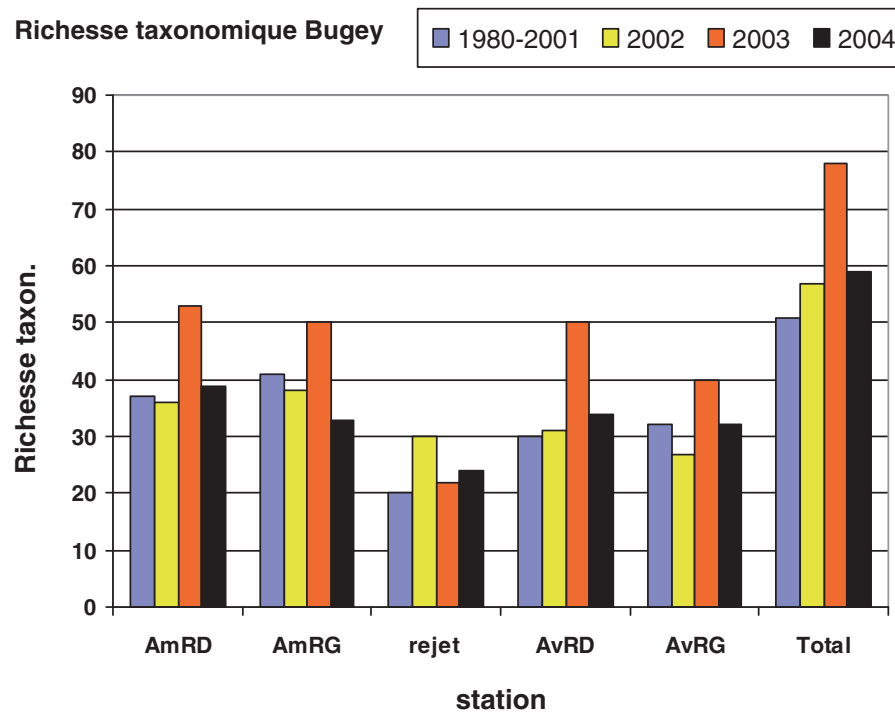

Fig. 12. Richesse taxonomique des macro-invertébrés échantillonnés sur des substrats artificiels en amont et en aval du CNPE de Bugey. Am : amont du CNPE. Av : aval du CNPE. RD, RG : rive droite, rive gauche.

Fig. 12. Taxonomic richness of macro-invertebrates sampled in artificial substrates upstream and downstream of Bugey NPP. Am: upstream the NPP. Av: downstream the NPP. RD, RG: right bank, left bank.

Tableau X. Comparaison de la richesse taxonomique moyenne de trois stations du site de Saint-Alban (Fruget, 2006) sur deux périodes. Localisation des stations sur la carte en annexe : SAL amont : 1, SAL aval proche : 2, Rhône court-circuité : 4.

Table X. Comparison of average taxonomic richness over two time periods at three sampling sites in the area of Saint-Alban (Fruget, 2006). See map in annex for the location of sampling sites: SAL amont: 1, SAL aval proche: 2, Rhône court-circuité: 4

\begin{tabular}{lccc}
\hline Période & $\begin{array}{c}\text { SAL } \\
\text { Amont }\end{array}$ & $\begin{array}{c}\text { SAL aval } \\
\text { proche }\end{array}$ & $\begin{array}{c}\text { Rhône } \\
\text { court-circuité }\end{array}$ \\
\hline $1985-1995$ & 24 & 20 & 22 \\
$1996-2004$ & 34 & 25 & 30 \\
\hline
\end{tabular}

également manifestée depuis 1996 (Fig. 13, Tab. X). Sur ce site, bien que les effectifs varient fortement d'une année à l'autre, une diminution des effectifs est observée depuis 2000 en raison de la chute d'abondance des crustacés Isopodes Asellidae concomitante au développement de l'Amphipode envahissant Dikerogammarus villosus; il faut toutefois remarquer que les effectifs varient fortement d'une année à l'autre (Dessaix \& Fruget, 2008).

\section{Analyse des chroniques de relevés \\ Les données collectées entre 1985 et 2004 en amont et en aval des quatre}



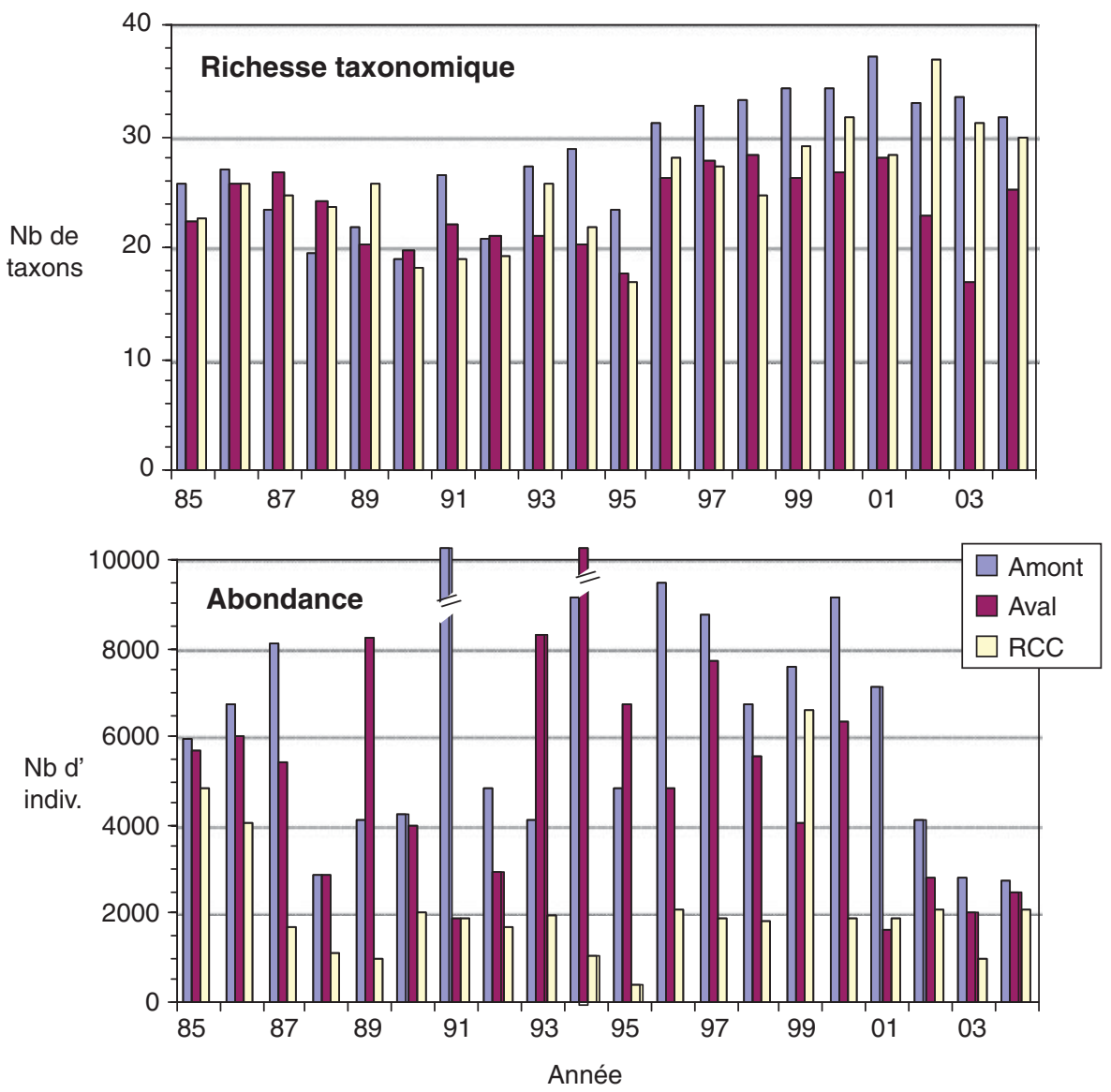

Fig. 13. Évolution de la richesse taxonomique et de l'abondance moyenne obtenue par substrat artificiel à l'amont, à l'aval du CNPE de Saint-Alban et dans le tronçon court-circuité (RCC). Localisation des stations sur la carte en annexe : SAL amont : 1, SAL aval proche : 2 , Rhône court-circuité : 4.

Fig. 13. Evolution of the taxonomic richness and the average abundance per unit artificial substrate at three sampling sites upstream (Amont) and downstream (Aval) of Saint-Alban NPP and in the by-passed section (RCC). See map in annex for the location of sampling sites.

CNPE (9 stations) ont été traitées par des analyses statistiques multitableaux (co-inertie multiple : ACOM), le niveau taxonomique étant la famille (Bady \& Fruget, 2006).

La méthode appliquée définit une structure de compromis représentative de l'ensemble des vingt années (Fig. 14) et des 9 stations.
Le site de Bugey, et notamment Bugey amont, se distingue cependant de l'ensemble des autres stations, par une plus grande richesse en taxons d'insectes.

La figure 14 montre l'existence de plusieurs ensembles distincts d'années, regroupés en trois périodes principales dans le tableau XI. 


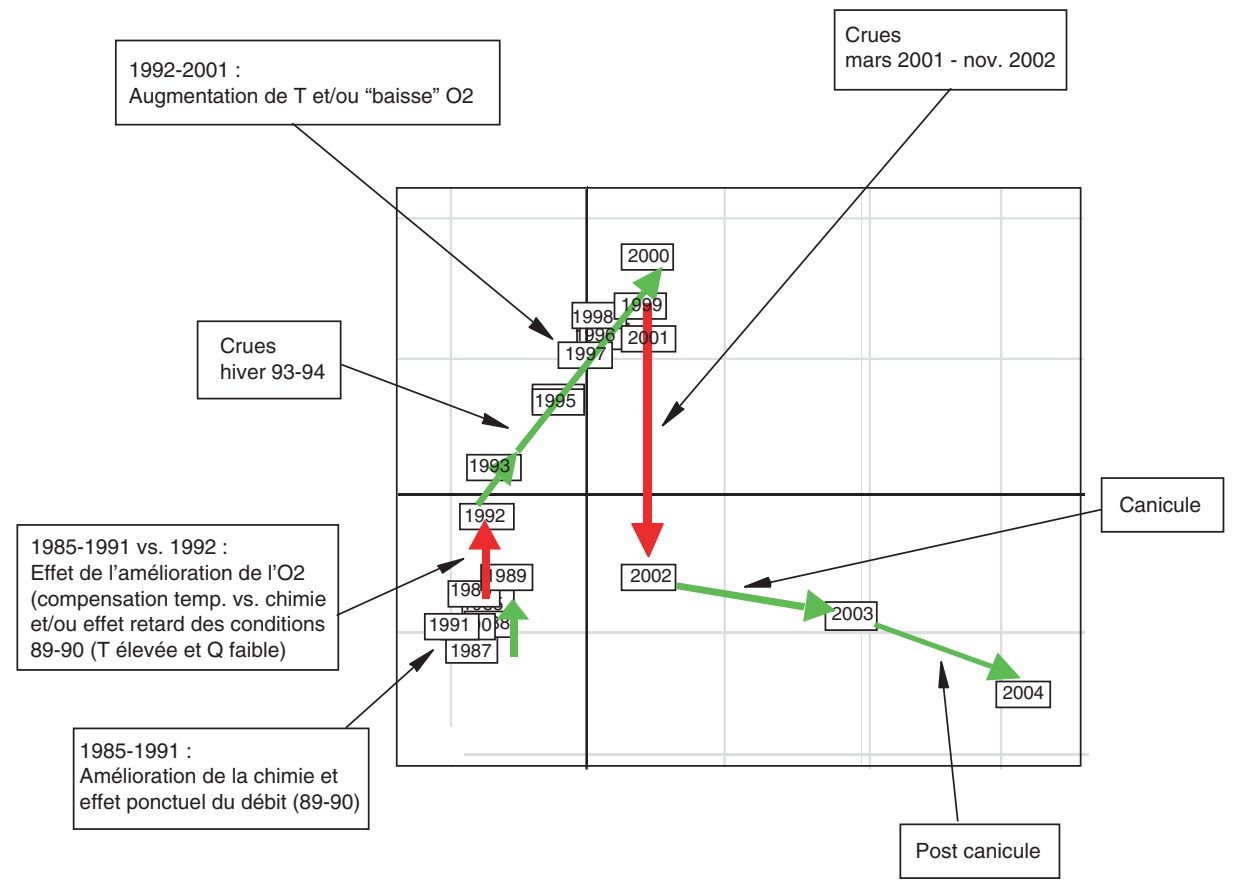

Fig. 14. Représentation de la structure temporelle de référence : les 20 années sur le premier plan factoriel du compromis (Bady \& Fruget, 2006).

Fig. 14. Diagram of the reference temporal structure resulting from a multiple co-inertia analysis: the 20 years are plotted against the first rank co-inertia axes (Bady \& Fruget, 2006).

Parmi les facteurs explicatifs, la qualité de l'eau doit être considérée au même titre que les facteurs thermique et hydrologique. La température et l'hydrologie influent sur la qualité d'eau, par exemple via des processus de dilution/concentration (débit), de déplacement des équilibres chimiques ou de modification de l'activité biologique (température) affectant le $\mathrm{pH}$ et l'oxygène dissous notamment.

En résumé, la première période 1985-1991 a été marquée par la variabilité chimique et hydrologique, tandis que la structure des peuplements a été peu modifiée. Au cours de la période 1992-2002, une dérive temporelle des conditions environnementales s'est instaurée, vraisemblablement liée à la thermie. C'est à partir de cette période que les peuplements ont le plus changé. La fin de la période (2003, année de la canicule et 2004) présente l'évolution la plus marquée.

L'étude de Daufresne et al. (2004), sur le site de Bugey a précisé les changements survenus sur le HautRhône de 1980 à 1999 :

- d'une part une disparition progressive des taxons d'eau froide et rhéophiles (préférant les forts 
Tableau XI. Les grands traits des évolutions de la faune macro-invertébrée et les évènements caractéristiques (Bady \& Fruget, 2006).

Table XI. Main characteristics of the evolution of the macro-invertebrate fauna and noteworthy events (Bady \& Fruget, 2006).

\begin{tabular}{ll}
\hline Périodes & Évènements caractéristiques et taxons structurant le peuplement \\
\hline 1985-1991 & Fortes concentrations en phosphate, azote ammoniacal et DBO5. \\
& Présence de Mollusques Bithyniidae, Physidae et Valvatidae (de Saint-Alban à \\
& Tricastin). Trichoptères Hydropsychidae à Tricastin. Trichoptères Rhyacophilidae \\
& et Plécoptères Chloroperlidae à Bugey. \\
& Fort étiage en 1989-1990. \\
& Achètes Piscicolidae et Mollusques Lymnaeidae (essentiellement à Cruas et \\
& Tricastin). \\
& 1990-1991 : Extension des Trichoptères Ecnomidae sur le Bas-Rhône \\
& 1992-1993 : Crue de l'hiver 93-94. \\
& Installation du Bivalve Corbicula fluminea. \\
& 1994-2001: Amélioration de la qualité de l'eau (phosphate, azote ammoniacal \\
& et DBO5) et augmentation progressive de la température. \\
& Trichoptère Ecnomidae Ecnomus tenellus, Mollusques Corbicula fluminea et \\
& Sphaeridae, Crustacés Asellidae (Bas-Rhône essentiellement) et des Ephé- \\
& mères Baetidae à Bugey. \\
& Minéralisation de l'eau et température plus élevée. Crues de mars 2001 et \\
novembre 2002. & Installation et extension de nouvelles espèces sur le Bas-Rhône (certaines \\
envahissantes) : crevette Atyaephyra desmarestii, Amphipode Gammaridae Di- \\
kerogammarus villosus, Polychète Hypania invalida. Augmentation générale des \\
Ephémères Heptageniidae (excepté Saint-Alban) et Caenidae, des Diptères \\
Cerapotogonidae (excepté Cruas) et des Trichoptères Hydroptilidae.
\end{tabular}

courants) tels que les Plécoptères (Chloroperla, Nemura, Protonemura, Amphinemura, Brachyptera), Trichoptères (Rhyacophila, Athripodes), Ephéméroptères (Ecdyonurus, Caenis), Diptères Stratyomiidae;

- d'autre part un développement de taxons thermophiles et lénitophiles (préférant les courants faibles) tels que les Hétéroptères (Corixa), Trichoptères (Oecetis), Diptères (Athricops, Lepidostoma), Odonates (Coenagrion, Platycnemis), Mollusques Gastéropodes
(Potamopyrgus Theodoxus) et bivalves (Corbicula), Planaires (Dendrocoelum)...

\subsubsection{Les espèces introduites}

La faune de macro-invertébrés du Rhône comporte 25 espèces exotiques dont 11 ont été introduites au cours de la dernière décennie. Dans la liste (Tab. XII) fournie par Bady \& Fruget (2006), plusieurs espèces sont connues comme envahissantes.

Ces espèces sont des compétiteurs d'espèces indigènes, c'est le 
Tableau XII. Liste des espèces exotiques d'invertébrés nouvelles dans le Rhône, les espèces apparues depuis 1993 sont notées en caractères gras (Bady \& Fruget, 2006).

Table XII. List of new exotic Invertebrates species in the Rhône River, the species identified since 1993 are indicated in bold characters (Bady \& Fruget, 2006).

\begin{tabular}{|c|c|c|c|}
\hline $\begin{array}{l}\text { Groupe } \\
\text { zoologique }\end{array}$ & Rhône & Bas-Rhône & Haut-Rhône \\
\hline Cnidaires & Craspedacusta sowerbyi & Cordylophora caspia & \\
\hline Bryozoaires & Pectinatella magnifica & Urnatella gracilis & \\
\hline Turbellariés & Dugesia tigrina & & \\
\hline Polychètes & Hypania invalida & & \\
\hline Oligochètes & Branchiura sowerbyi & & \\
\hline Gastéropodes & $\begin{array}{c}\text { Menetus dilatatus } \\
\text { Physa acuta } \\
\text { Gyraulus parvus } \\
\text { Potamopyrgus } \\
\text { antipodarum }\end{array}$ & Lithoglyphus naticoïdes & \\
\hline Bivalves & $\begin{array}{c}\text { Dreissena polymorpha } \\
\text { Corbicula fluminea }\end{array}$ & & \\
\hline Crustacés & $\begin{array}{c}\text { Atyaephyra desmarestii } \\
\text { Gammarus roeseli } \\
\text { Orchestia cavimana } \\
\text { Orconectes limosus }\end{array}$ & $\begin{array}{c}\text { Chelicorophium curvispinum } \\
\text { Crangonyx pseudogracilis } \\
\text { Dikerogammarus villosus } \\
\text { Gammarus tigrinus } \\
\text { Hemimysis anomala } \\
\text { Jaera istri } \\
\text { Procambarus clarkii }\end{array}$ & $\begin{array}{l}\text { Pacifastacus } \\
\text { leniusculus }\end{array}$ \\
\hline
\end{tabular}

cas notamment chez les Amphipodes (cf. Dessaix \& Fruget, 2008). Elles peuvent également modifier les réseaux trophiques en éliminant le plancton végétal et influencer la qualité de l'eau en filtrant des particules nutritives en suspension (Mollusque Corbicula).

Contrôle de la biomasse phytoplanctonique par le bivalve Corbicula

La chlorophylle a est l'indicateur le plus couramment utilisé pour quantifier la biomasse des algues planctoniques dans les eaux douces et marines. Sur la chronique des concentrations en chlorophylle a dans le Rhône à Bugey (Fig. 15a), trois périodes distinctes sont visibles, caractérisées par:

- de faibles biomasses entre 1979 et 1984 (maximum annuel $<10 \mu \mathrm{g} / \mathrm{L}$ ),

- des biomasses plus élevées entre 1985 et 1990 (maximum annuel de 10 à $50 \mu \mathrm{g} / \mathrm{L})$,

- le retour aux faibles biomasses de la première période de 1991 à 2003. 


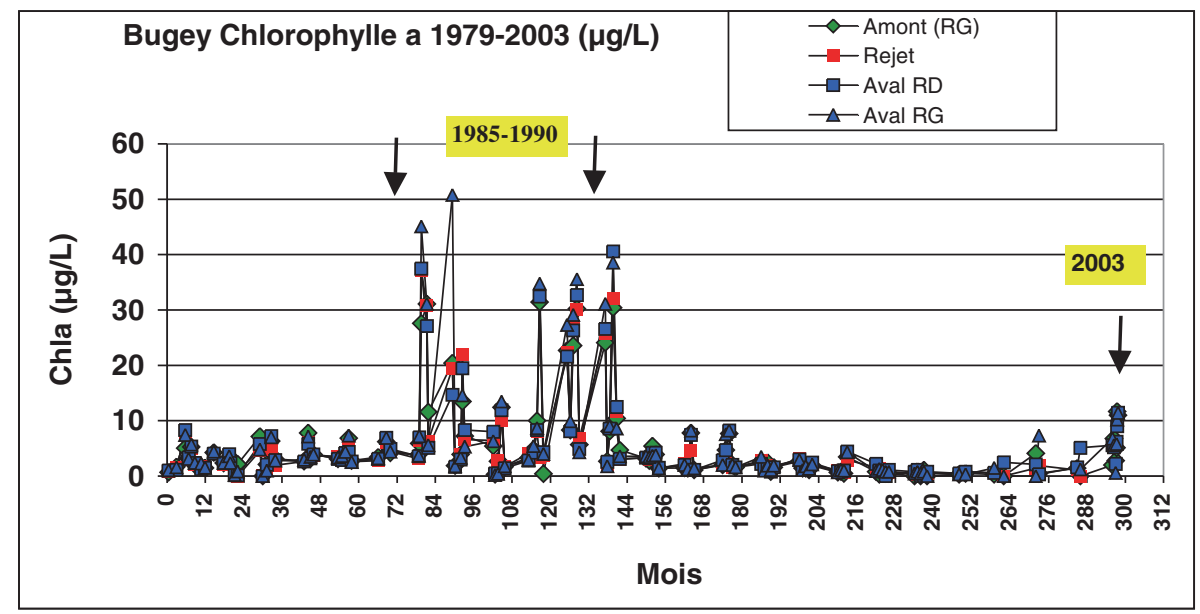

(a)

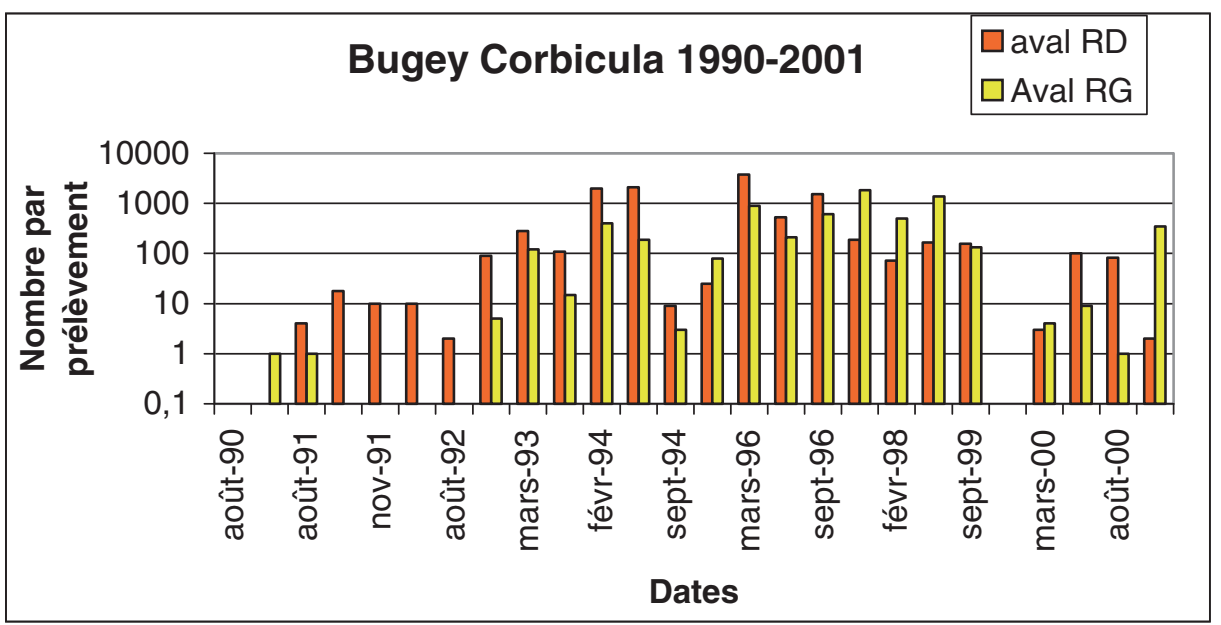

(b)

Fig. 15. (a) Chronique de la biomasse du phytoplancton (chlorophylle a) dans le Rhône sur le site de Bugey. (b) Abondance du bivalve Corbicula depuis l'introduction de l'espèce en 1990 dans le Rhône sur le site de Bugey.

Fig. 15. (a) Time series of phytoplankton biomass (chlorophyll a) in the Rhône River at Bugey. (b) Abundance of the Corbicula bivalve since the introduction of this species in 1990 in the Rhone River in the Bugey area. 

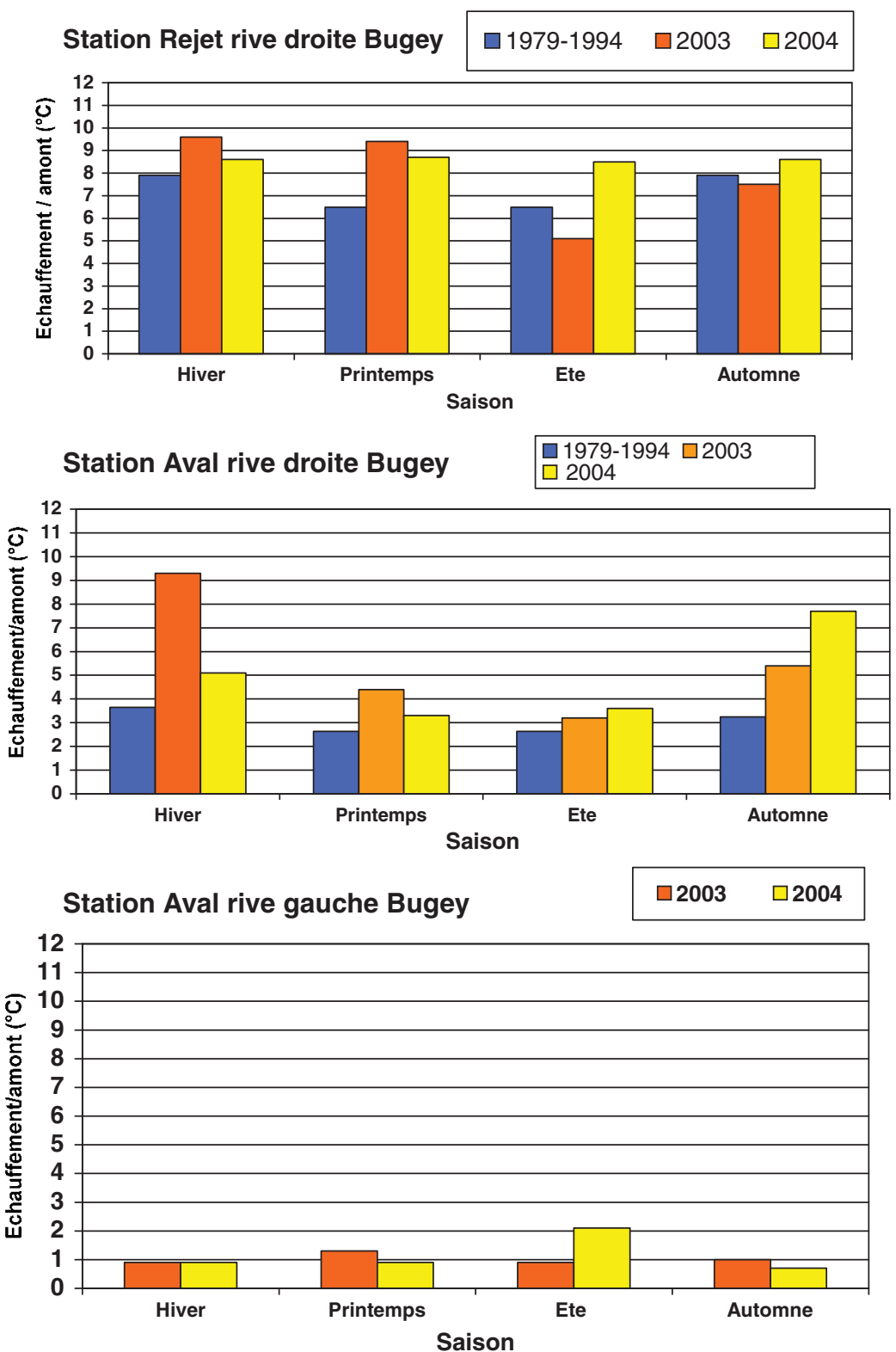

Fig. 16. Échauffement saisonnier entre amont et aval aux trois stations échantillonnées en aval du CNPE de Bugey. Données de Ginot et al. (1996) pour la période 1979-1994. Données Cemagref Lyon pour les années 2003 et 2004.

Fig. 16. Seasonal upstream-downstream temperature rise at three sampling sites downstream of Bugey NPP. Data in Ginot et al. (1996) for the 1979-1994 period. CEMAGREF data for the year 2003 and 2004. 
De telles modifications de la teneur en chlorophylle planctonique traduisent généralement un changement fonctionnel significatif, l'augmentation de biomasse étant un signe d'eutrophisation.

Deux causes peuvent être évoquées pour expliquer l'augmentation très nette de la biomasse. La mise en service de l'aménagement de SaultBrénaz en 1986 a entraîné un ralentissement de l'eau susceptible de favoriser la croissance du phytoplancton et la mise en eau du réservoir a probablement augmenté les apports en sels nutritifs issus du lessivage du sol inondé.

Pour expliquer la chute brutale de la biomasse phytoplanctonique à partir de 1991, deux causes interviennent conjointement : la réduction des apports en phosphates et le développement important du bivalve Corbicula fluminea, une espèce exotique apparue dans le Haut-Rhône en 1990 (Fig. 15b). Cette espèce envahissante est capable de consommer la plus grande partie du phytoplancton dans les grandes rivières et dans les lacs.

\subsubsection{Effets des rejets thermiques}

\section{Étude sur le site de Bugey}

Sur ce site, le panache d'eau échauffée des tranches en circuit ouvert du CNPE suit la rive droite jusqu'au confluent avec l'Ain. Les échauffements décroissent progressivement du rejet vers l'aval (Fig. 16, Tab. XIII). Une variation saisonnière est particulièrement marquée à la station 3 en rive droite : l'échauffement estival est moins fort en raison du débit élevé pendant cette période. La station 4, située en rive gauche, conserve en partie les caractéristiques thermiques du Rhône amont et est moins échauffée que le Rhône après la zone de mélange.

L'existence de relevés de macroinvertébrés par substrats artificiels placés dans cette section du fleuve à l'aval du rejet thermique a permis de mettre en valeur les changements biologiques s'opérant dans la zone de diffusion du panache.

Les données obtenues en 2003 et 2004 (Roger et al., 2004, 2005), présentent un intérêt particulier car ces deux années sont caractérisées par des conditions climatiques (fortes températures et faibles débits) qui pourraient se rapprocher de la situation moyenne prévue vers la fin du $\mathrm{XXI}^{\mathrm{e}}$ siècle.

En 2003 et 2004 (Fig. 17), la richesse taxonomique et l'indice de qualité biologique ont été stables dans la gamme des échauffements $\mathrm{de}+1^{\circ} \mathrm{C}$ à $+6^{\circ} \mathrm{C}$ (stations 4 en rive gauche et 3 en rive droite). En

Tableau XIII. Échauffements moyens mesurés aux stations aval du CNPE de Bugey.

Table XIII. Average temperature elevation measured at three sampling sites downstream of Bugey NPP.

\begin{tabular}{|c|c|c|c|c|c|}
\hline \multirow[b]{2}{*}{ Période } & \multicolumn{3}{|c|}{ Échauffement moyen mesuré } & \multicolumn{2}{|c|}{ Échauffement après mélange } \\
\hline & Station Rejet RD & Station 3 RD & Station $4 \mathrm{RG}$ & Fréquence $50 \%$ & Fréquence $90 \%$ \\
\hline 1979-1994 & $7,2^{\circ} \mathrm{C}$ & $3,0^{\circ} \mathrm{C}$ & $0,6^{\circ} \mathrm{C}^{*}$ & & \\
\hline 2003 & $7,3^{\circ} \mathrm{C}$ & $3,9^{\circ} \mathrm{C}$ & $1,0^{\circ} \mathrm{C}$ & & \\
\hline 2004 & $8,6^{\circ} \mathrm{C}$ & $4,9^{\circ} \mathrm{C}$ & $1,2^{\circ} \mathrm{C}$ & & \\
\hline 1991-2003 & & & & $1,5^{\circ} \mathrm{C}$ & $3,2^{\circ} \mathrm{C}$ \\
\hline
\end{tabular}

*moyenne de 16 mesures entre 1978 et 1989 (Roger et al., 1990). 


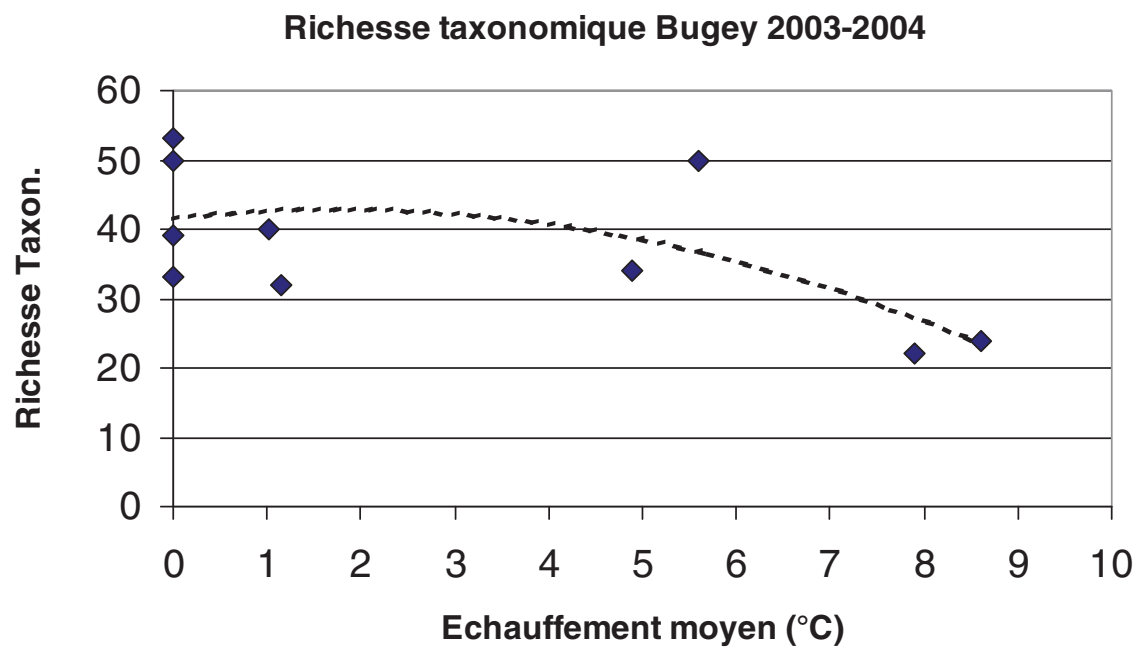

Indice biologique IQBP Bugey 2003-2004
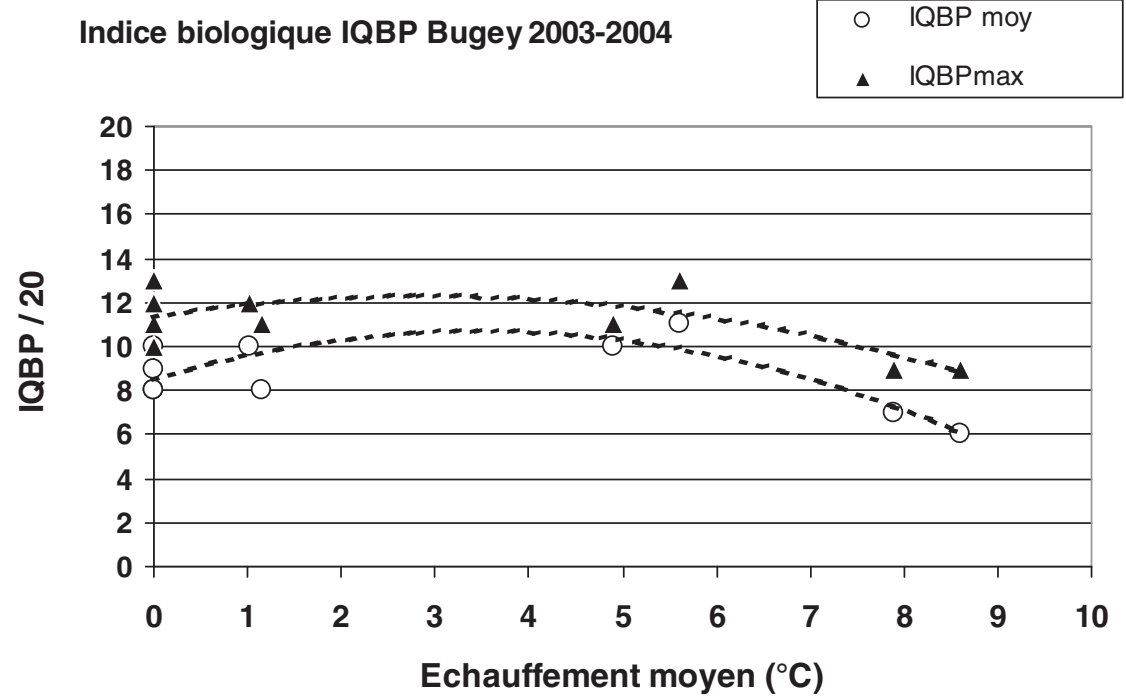

Fig. 17. Richesse taxonomique et indice de qualité biologique potentielle (IQBP) représentés en fonction de l'échauffement moyen aux stations amont et aval du CNPE de Bugey en 2003 et 2004.

Fig. 17. Evolution of species richness and biological quality index (IQBP) as a function of temperature rise upstream-downstream of Bugey power plant in 2003 and 2004. 
revanche, une chute de ces deux descripteurs du peuplement a été observée à la station « rejet » où l'échauffement moyen est de l'ordre de $+8^{\circ} \mathrm{C}$ à $+9^{\circ} \mathrm{C}$.

Trois taxons dominent très largement le peuplement de macroinvertébrés de toutes les stations: les Crustacés Amphipodes Gammaridés, les larves de Diptères Chironomidés et les Annélides Oligochètes. Les Gammaridés présentent des biomasses très élevées mais aussi très variables, probablement en raison de la sensibilité thermique de ce groupe chez lequel les abondances diminuent lorsque la température excède $20^{\circ} \mathrm{C}$ (Roger et al., 2004).

D'autres taxons plus thermophiles, comme le ver Planaire Dugesia tigrina, sont plus abondants au moment du maximum thermique. Enfin, des espèces préférant les écoulements lents sont apparues en 2003 dans le Rhône à Bugey : il s'agit des Trichoptères Ecnomidae (Ecnomus tenelIus), Polycentropodidae (Cyrnus trimaculatus), Leptoceridae (Oecetis). En 2004, seul Oecetis a été retrouvé sur le site.

Sur les sites du Bas-Rhône (Bady \& Fruget, 2006; Fruget, 2006), une chute de richesse spécifique a été observée dans les stations proches des rejets par rapport à l'amont (Fig. 12). À Saint-Alban, où l'échauffement moyen est d'environ $5^{\circ} \mathrm{C}$ entre la station de référence amont (pk 47) et la station en aval proche (pk 49), la baisse de richesse entre les prélèvements unitaires (substrats artificiels) de chaque station passe de $5 \%$ pour la période 1985-1995 à $24 \%$ pour la période 1996-2004.

Conjointement à l'échauffement de l'eau aux stations en aval des CNPE, d'autres facteurs concourent probablement à l'explication de ces écarts. À Bugey par exemple, la diversité des conditions d'habitat, plus grande à l'amont qu'à l'aval du CNPE, peut expliquer en partie l'écart négatif constaté entre aval et amont.

\subsubsection{Conclusions}

II existe un gradient longitudinal de richesse taxonomique depuis Bugey, site le plus riche notamment en familles d'insectes, jusqu'à Tricastin, site appauvri d'environ $25 \%$ par rapport à Bugey.

Sur le Haut-Rhône, les relevés effectués entre 1975 et 1988 révèlent une baisse de la richesse taxonomique des insectes. L'étude statistique réalisée sur les données de 1980 à 1999 montre la disparition progressive des taxons d'eau froide préférant les forts courants et leur remplacement par des taxons thermophiles préférant les courants faibles. Ce changement dans la structure du peuplement, qui concerne aussi bien les stations situées en amont qu'en aval du CNPE, peut être mis en relation d'une part avec le changement climatique et d'autre part avec l'aménagement hydraulique du fleuve.

Sur le Bas-Rhône, une évolution temporelle assez comparable du peuplement de macro-invertébrés se manifeste sur l'ensemble des sites, avec un décalage dans le temps. La dernière période (2000-2004) est marquée par l'influence des espèces exotiques et invasives introduites dans les années 1990, avec un impact sur les crustacés autochtones qui pourrait être important. 
Effet des rejets thermiques des CNPE. Dans les canaux de rejets des CNPE ou à proximité immédiate des rejets thermiques, la richesse taxonomique chute systématiquement par rapport à l'amont. À Bugey, cela correspond à un échauffement permanent de $+8^{\circ} \mathrm{C}$ à $+9^{\circ} \mathrm{C}$. En 2003 et 2004, dans les stations en aval de Bugey où l'échauffement atteint $+1^{\circ} \mathrm{C}$ à $+6^{\circ} \mathrm{C}$, la richesse taxonomique et l'indice de qualité biologique sont restés stables par rapport à l'amont. À Saint-Alban, la richesse unitaire de la station aval proche, échauffée d'environ $+5^{\circ} \mathrm{C}$, est en moyenne de $15 \%$ inférieure à celle de la station amont. Une baisse modérée de la richesse taxonomique a été observée à Cruas et Tricastin par rapport à l'amont. Des causes autres que la température, comme les différences stationnelles, pourraient également expliquer en partie ces écarts.

Depuis 1991, et l'installation du bivalve Corbicula, le peuplement de macro-invertébrés est de plus en plus influencé par la présence d'espèces exotiques.

\subsection{Macrophytes}

Les observations réalisées depuis 1985 dans la retenue de Péagede-Roussillon sur les phanérogames aquatiques en amont et en aval du CNPE de Saint-Alban permettent de suivre la richesse spécifique de ce peuplement et fournissent des données semi-quantitatives sur l'abondance des macrophytes aquatiques (Fruget, 2006).

Ces relevés sont effectués sur une bande parallèle à la rive à l'amont et à l'aval du CNPE. Le rejet du CNPE sur la rive gauche a tendance à s'écarter de la rive puis à s'en rapprocher sous l'effet du courant dirigé vers l'entrée du canal de dérivation de l'usineécluse. II est ainsi possible de comparer les caractéristiques quantitatives et qualitatives du peuplement sur quatre sites :

- l'amont du CNPE en rive gauche (pk 46-47), sans échauffement provenant du CNPE,

- l'aval du CNPE en rive droite (pk 49-50), à échauffement faible et intermittent (environ $+1,5^{\circ} \mathrm{C}$ ),

- l'aval proche du CNPE en rive gauche (pk 49-50), à échauffement permanent de $+5^{\circ} \mathrm{C}$,

- l'aval éloigné du CNPE en rive gauche (pk 50-51), à échauffement permanent moyen de $+2,3^{\circ} \mathrm{C}$.

Tous sites confondus, la richesse spécifique des phanérogames a diminué de 13 à 7 espèces entre 1985 et 1990 puis ré-augmenté pour atteindre 15 espèces en 2003 et 11 espèces en 2004.

La chronique de la richesse spécifique annuelle (Fig. 18) montre deux particularités :

- le site aval éloigné en rive gauche est le plus souvent moins riche que les trois autres,

- le site aval proche en rive gauche se distingue depuis 1998 par une richesse supérieure à celle des trois autres (Tab. XIV).

Ainsi, de manière récurrente depuis plusieurs années, et ce, en dépit d'un mélange plus effectif du rejet, la richesse floristique et l'abondance sont nettement moindres à la station aval éloignée en rive gauche. L'écart de richesse floristique par rapport à la zone aval proche en rive gauche a même 


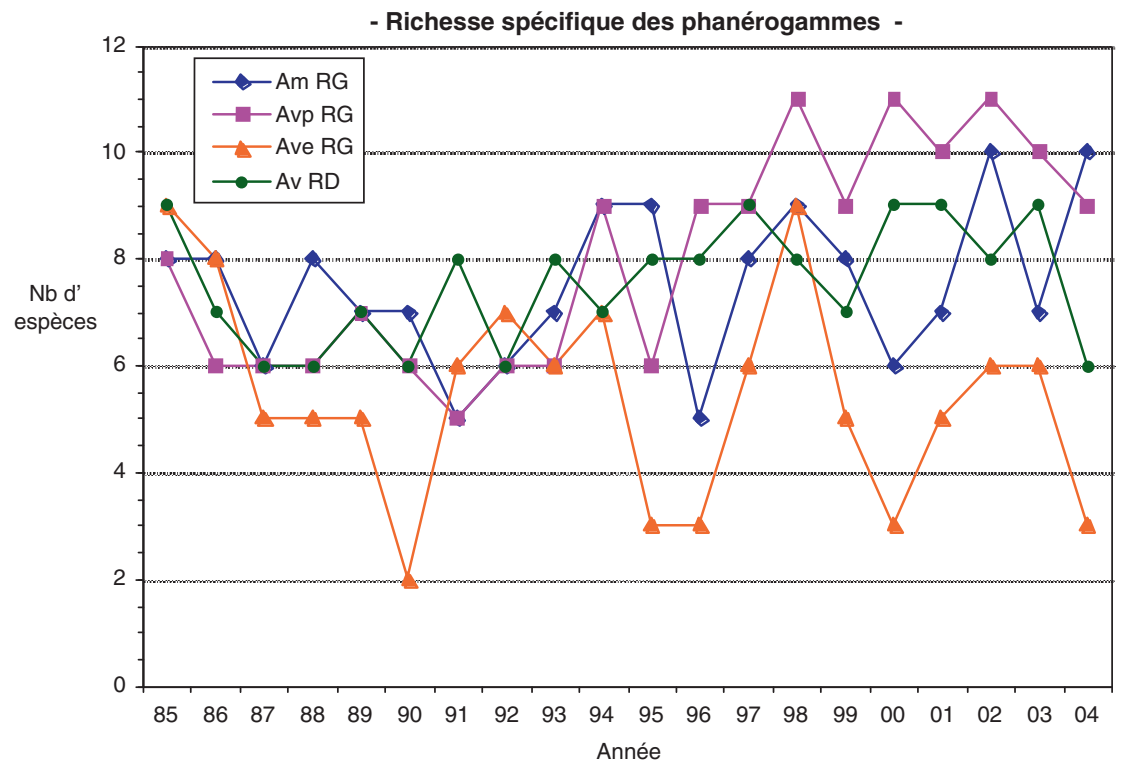

Fig. 18. Richesse spécifique moyenne du peuplement de phanérogames aquatiques échantillonnées sur quatre stations du suivi hydrobiologique du CNPE de Saint-Alban (Fruget, 2006). Les codes sont donnés dans le tableau XIV.

Fig. 18. Average species richness of aquatic phanerogames recorded at the four Saint-Alban NPP monitoring sites (Fruget, 2006). Codes are given in Table XIV.

Tableau XIV. Richesse spécifique moyenne du peuplement de phanérogames aquatiques échantillonnées pendant deux périodes en quatre sites à l'amont et à l'aval du CNPE de Saint-Alban (Fruget, 2006). Am : amont, Avp : aval proche, Ave : aval éloigné, RD : Rive droite, RG : Rive gauche.

Table XIV. Average species richness of aquatic phanerogames recorded during two periods upstream and downstream of the Saint-Alban NPP. Am: upstream, Avp: downstream close to NPP, Ave: downstream further from NPP, RD: right bank, $R G$ : left bank

Am RG Avp RG Ave RG AvRD

\begin{tabular}{ccccc}
\hline $1985-1994$ & 7 & 7 & 6 & 7 \\
$1995-2004$ & 8 & 10 & 5 & 8
\end{tabular}

été multiplié par 5 entre les périodes 1985-1994 et 1995-2004 (cf. Tab. XIV).

Malgré la grande homogénéité de la structure rivulaire le long de la retenue (enrochements des berges et banquettes limoneuses), le peuplement de macrophytes semble ainsi défavorisé par la combinaison de deux facteurs : d'une part le facteur thermique avec un échauffement permanent compris entre $2^{\circ} \mathrm{C}$ et $5^{\circ} \mathrm{C}$, d'autre part le caractère morphodynamique de cette zone à l'extrémité aval de la retenue (rupture de pente plus proche de la rive et zone d'implantation plus limitée afin d'éviter le batillage, accroissement de la vitesse du courant). 
Le facteur thermique semble prendre une part essentielle dans l'explication de ces évolutions.

En conclusion, dans l'hypothèse où seul le facteur thermique intervient dans la structure du peuplement, ces données indiquent que la diversité des macrophytes est peu affectée par un échauffement permanent de $+5^{\circ} \mathrm{C}$ (Avp RG), même si une réduction de la variété et de l'abondance des peuplements est observée localement à l'aval du CNPE (Ave RG).

\subsection{Poissons}

\subsubsection{Structure spatiale du peuplement de poissons et aménagements du Rhône}

Au milieu du $X X^{e}$ siècle, la rupture de la continuité engendrée par la construction des barrages sur le Haut-Rhône, la création de nouveaux milieux (retenues et canaux) et la vidange périodique des retenues ont entraîné un déclin des Salmonidés et des Cyprinidés d'eau vive ainsi que la disparition de l'apron. Les Cyprinidés euryèces (gardon, goujon, chevaine) dominent dans les secteurs aménagés; des espèces rhéophiles se maintiennent toutefois dans les tronçons court-circuités (barbeau fluviatile, vandoise, spirlin et Salmonidés) et sont présentes dans le secteur du Bugey (Fruget, 2003).

Sur le Bas-Rhône, le peuplement originel composé de Salmonidés, de Cyprinidés d'eau vive et comportant des migrateurs amphihalins (lamproie marine, alose et anguille) a évolué après les aménagements vers un peuplement dominé par les Cyprinidés d'eau calme (gardon, ablette) et la quasi-disparition des espèces migratrices (Carrel et al., 1995). Actuellement, une nette différence oppose ainsi le secteur du HautRhône à Bugey qui conserve certains caractères originels sur le plan morpho-dynamique (vitesse rapide, persistance de substrat grossier) au Bas-Rhône transformé en chenal dimensionné pour la navigation à grand gabarit, différence apparente dans la structure des peuplements de poissons.

La figure 19 illustre les différences relevées en 2004 et 2005 entre le peuplement de poissons du Haut-Rhône à Bugey et celui du Bas-Rhône.

Le peuplement du site de Bugey est caractérisé par cinq espèces dont les abondances et les occurrences sont nettement plus importantes que dans les autres sites : le spirlin, la vandoise, le barbeau fluviatile, le vairon et le blageon. Les espèces thermophiles et lénitophiles apparaissent comme représentatives des sites du BasRhône (gardon, ablette, brème bordelière, perche-soleil, poisson-chat, brème commune, rotengle et tanche). L'anguille est également beaucoup plus largement échantillonnée dans le Bas-Rhône que dans le Haut- Rhône.

Dans ce contexte aménagé, les sections court-circuitées jouent un rôle prépondérant dans le maintien de nombreuses populations, en particulier pour les espèces se reproduisant sur des substrats grossiers (graviers, galets) ou des seuils naturels. Les espèces les plus lentiques, utilisant souvent un support végétal pour déposer leur ponte, trouvent dans les sections aménagées des conditions propices à leur reproduction.

II faut enfin signaler le rôle des annexes hydrauliques latérales artificielles, telles que les caissons 

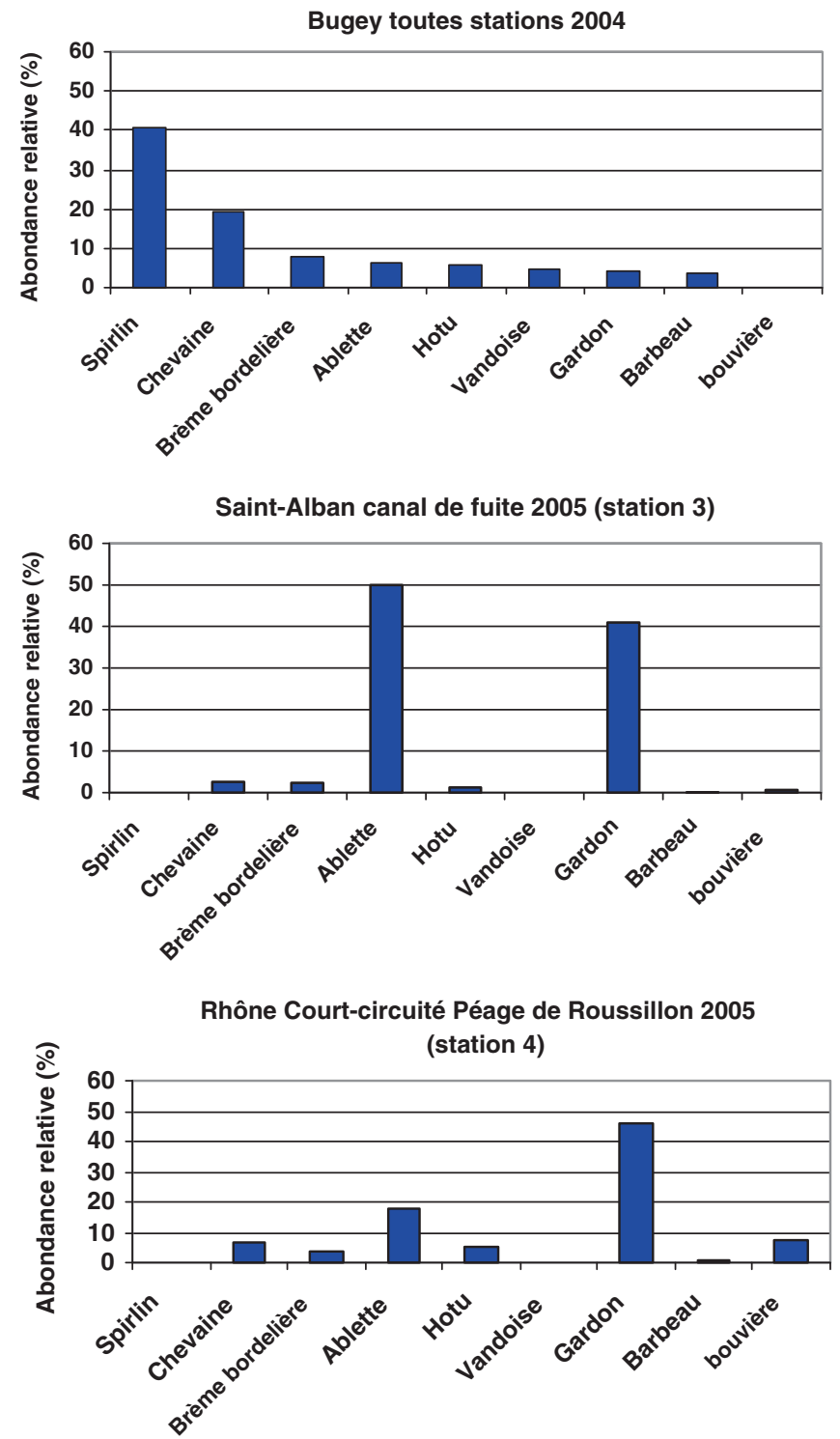

Fig. 19. Abondances relatives des principales espèces de poissons échantillonnées à Bugey, à SaintAlban à l'extrémité du canal de fuite et dans le tronçon court-circuité de l'aménagement de Péagede-Roussillon.

Fig. 19. Strength of the main fish species collected near Bugey, at the end of the outlet canal in Saint-Alban, and in the by-passed section of the Péage-de-Roussillon scheme. 
délimités par les digues submersibles dans le bas Rhône, qui par leur richesse en juvéniles de poissons jouent un rôle important dans le recrutement de plusieurs espèces sur ce secteur (Nicolas \& Pont, 1995).

\section{Les espèces introduites}

Les causes des introductions d'espèces listées dans le tableau XV sont l'aquaculture, les introductions volontaires, la colonisation à partir des réseaux de canaux.

Depuis la fin des années 1980, trois espèces exotiques sont apparues sur l'axe rhodanien :

- le pseudorasbora, Pseudorasbora parva (Cyprinidé), sur les sites du Bas-Rhône (à partir de 1989) et de Bugey (à partir de 1993),

- le carassin argenté, Carassius auratus gibelio (Cyprinidé), sur les sites du Bas-Rhône en 1989,

- le silure en 1987 sur le Bas-Rhône et en 1997 sur le site de Bugey.

Le succès de ces introductions pourrait être lié au changement climatique.
II pourrait notamment favoriser l'expansion du silure (Daufresne et al., 2005). Les conséquences de l'expansion du silure restent à établir. II peut en effet exercer une forte pression de prédation et agir en tant que compétiteur sur les espèces carnassières.

\subsection{2 Évolution du peuplement et changement climatique}

Traitement des données recueillies sur les sites de CNPE du Rhône

II est largement admis par la communauté scientifique que la température moyenne planétaire a augmenté d'environ $+0,74^{\circ} \mathrm{C}$ au cours des années 1906-2005 (IPCC, 2007). Toute recherche sur le régime thermique des cours d'eau doit aujourd'hui prendre en compte la dérive climatique observée au cours des dernières décennies. C'est en ce sens qu'une première étude a été réalisée par le Cemagref, groupement de Lyon, à partir des données collectées dans le cadre de la surveillance hydro-écologique du CNPE de Bugey

Tableau XV. Liste des espèces de poissons introduites dans le bassin du Rhône (Carrel, 2002).

Table XV. List of fish species introduced to the Rhône river basin (Carrel, 2002).

\begin{tabular}{|c|c|c|c|}
\hline Période d'introduction & Famille & Nom français & Nom latin \\
\hline 1880’s & Salmonidae & Truite arc en ciel & Onchorhynchus mykiss \\
\hline $\begin{array}{c}\text { Fin du } X I X^{e} \text { siècle et début } \\
\text { du } X X^{e} \text { siècle }\end{array}$ & Salmonidae & Saumon de fontaine & Salvelinus fontinalis \\
\hline 1860 's & Percidae & Grémille & Gymnocephalus cernua \\
\hline 1880’s & Cyprinidae & Hotu & Chondrostoma nasus \\
\hline 1920's & Centrarchidae & Perche soleil & Lepomis gibbosus \\
\hline 1920’s & Ictaluridae & Poisson-chat & Ameirus melas \\
\hline 1930's & Percidae & Sandre & Stizostedion lucioperca \\
\hline 1930's & Poeciliidae & Gambusie & Gambusia affinis \\
\hline 1940's & Centrarchidae & Black bass & Micropterus salmoides \\
\hline Depuis $1956 ?$ & Cyprinidae & Able de Heckel & Leucaspius delineatus \\
\hline 1987 & Siluridae & Silure & Silurus glanis \\
\hline 1989 & Cyprinidae & Pseudorasbora & Pseudorasbora parva \\
\hline 1989 & Cyprinidae & Carassin doré & Carassius auratus gibelio \\
\hline
\end{tabular}


Tableau XVI. Classement des principales espèces de poissons présentes dans le Rhône selon un indice de thermophilie défini par Daufresne et Boët (2005).

Table XVI. Classification of the Rhone river main fish species according to an affinity for high temperatures index defined by Daufresne and Boët (2005).

\begin{tabular}{lclclc}
\hline \multicolumn{1}{c}{ Espèce } & $\begin{array}{c}\text { Indice de } \\
\text { thermophilie }\end{array}$ & Espèce & $\begin{array}{c}\text { Indice de } \\
\text { thermophilie }\end{array}$ & Espèce & $\begin{array}{c}\text { Indice de } \\
\text { thermophilie }\end{array}$ \\
\hline Brème bordelière & 1 & Ablette & 0,75 & Brochet & 0,25 \\
Brème & 1 & Barbeau & 0,75 & Chevaine & 0,25 \\
Poisson-chat & 1 & Bouvière & 0,75 & Goujon & 0,25 \\
Rotengle & 1 & Gardon & 0,75 & Perche & 0,25 \\
Silure & 1 & Spirlin & 0,75 & Perche soleil & 0,25 \\
Tanche & 1 & & & Hotu & 0 \\
& & & & Vandoise & 0 \\
\hline
\end{tabular}

depuis 1979 (Daufresne et al., 2004). Dans le cadre du groupe de travail "Thermie et Hydrobiologie », constitué à l'initiative d'EDF et du Cemagref, puis de la phase III de l'étude thermique du Rhône, ce travail a été repris, étendu aux autres CNPE du Rhône et actualisé en utilisant les observations faites jusqu'en 2003 (Daufresne \& Boët, 2005) et 2004 (Daufresne et al., 2005).

Pour étudier statistiquement les effets de l'échauffement par les CNPE en prenant en compte l'augmentation de la température attribuable au changement climatique, Daufresne et
Boët (2005) ont affecté aux principales espèces de poissons un indice de thermophilie qui caractérise leur préférence thermique. L'indice varie entre 0 et 1 ; il est maximum pour les poissons thermophiles et minimum pour les espèces d'eau plus froide (Tab. XVI).

Parallèlement à l'élévation des températures annuelles moyennes, le traitement statistique (analyse factorielle des correspondances) a mis en évidence une évolution de la structure des peuplements de poissons sur les quatre sites de CNPE du Rhône (Fig. 20). Cette évolution se caractérise par la régression des " espèces

Fig. 20. [en pages suivantes] Colonne de gauche : chronique des coordonnées factorielles de l'AFC des pêches sur les quatre sites de CNPE sur le Rhône (Daufresne et al., 2005), la première année est 1979. La contribution des principales espèces et les traits écologiques vis-à-vis de la thermie sont représentés à droite des diagrammes. Les valeurs correspondant aux données moyennées sur toutes les stations de pêches d'un site sont en trait plein, celles relatives aux stations en amont des CNPE sont représentées par des carrés ouverts, celles relatives aux stations en aval des CNPE sont représentées par des triangles ouverts. Colonne de droite : chronique des températures moyennes annuelles relevées en amont des quatre sites de CNPE.

Fig. 20. [see next pages] Left column: annual mean scores (solid line: all sampling sites pooled) of samples and species scores on COA axes for the 4 study areas near nuclear power plants on the Rhone river (First year of study is 1979). For each study area, only the first axis with a significant temporal trend is shown. Temporal trends in sampling sites located upstream (open squares) and downstream (open triangles) from the nuclear power plant are represented. Right column : mean annual temperature series recorded upstream of the 4 NPP sites. 

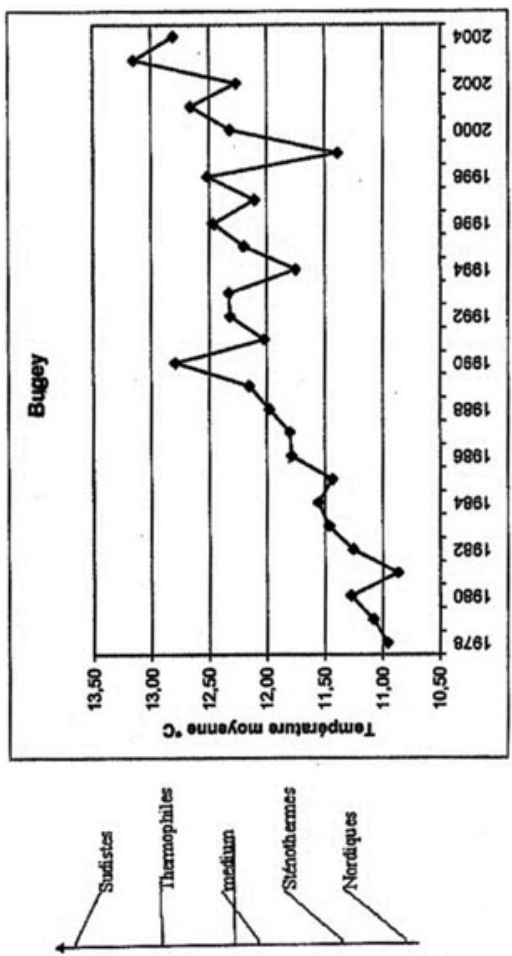

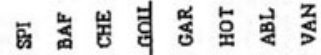
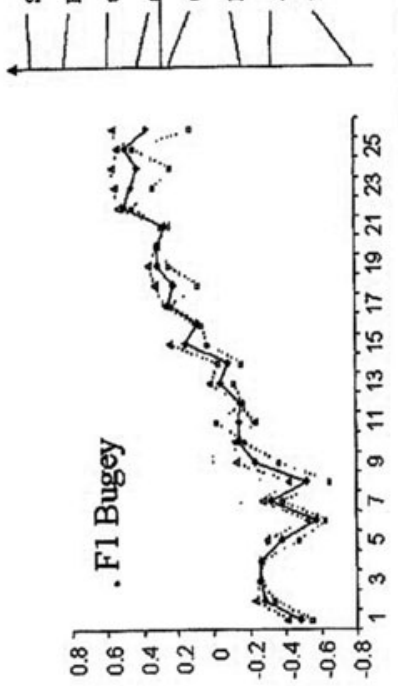
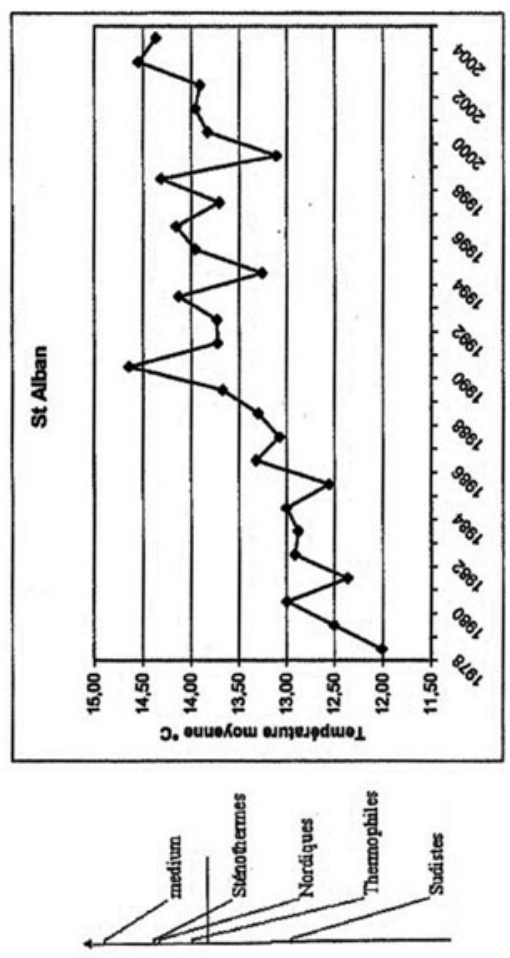

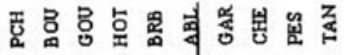
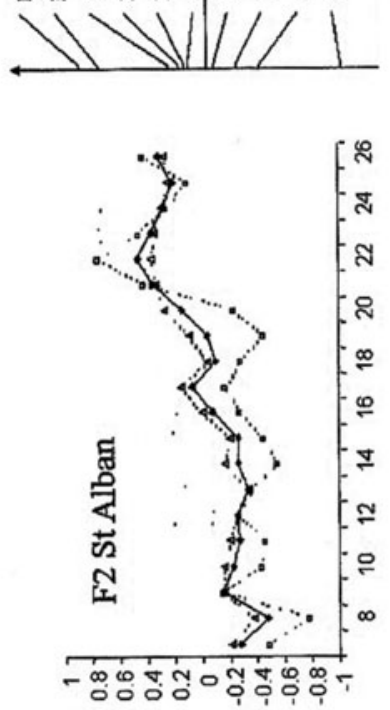

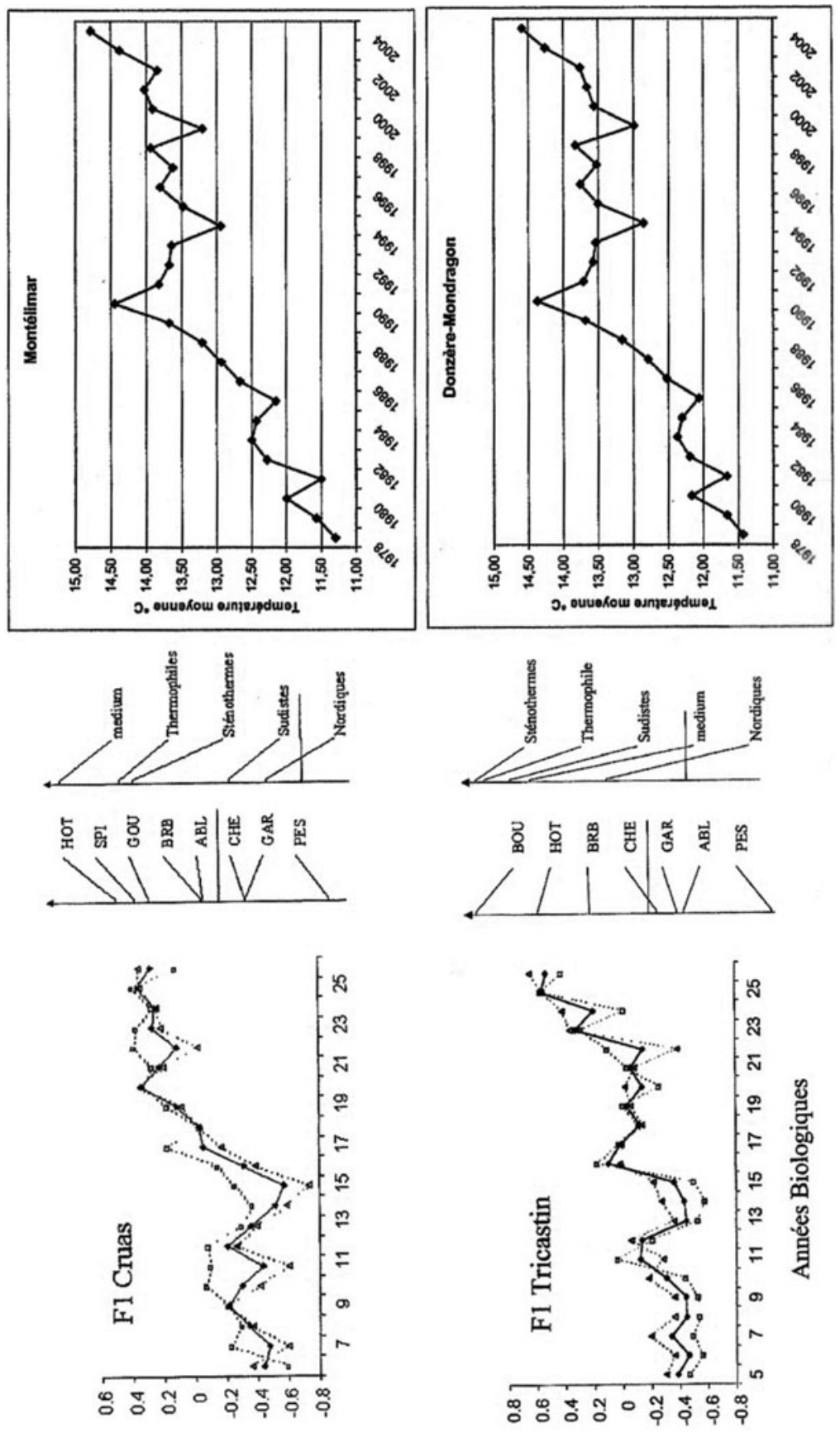

ลิ่
कo 
d'eau froide » au profit des espèces plus thermophiles. Elle est plus marquée sur le Haut-Rhône qu'à l'aval de Lyon.

Sur le Haut-Rhône, à Bugey, le peuplement a réagi le plus nettement à l'élévation tendancielle de la température par une dérive vers les espèces thermophiles : le spirlin, le chevaine et le barbeau remplacent progressivement la vandoise parmi les espèces dominantes. L'ombre commun n'est plus capturé depuis 2001. Chez les espèces marginales de ce peuplement, des changements ont aussi été notés : la brème bordelière, la loche franche et le silure sont en expansion alors que le brochet régresse.

Dans la mesure où les communautés piscicoles en aval de Lyon sont constituées d'espèces plus thermophiles, les modifications liées au changement climatique sont moins marquées que sur le Haut-Rhône, elles sont cependant visibles. Ce sont des évènements d'ordre hydrologique : étiages sévères et crues remarquables, comme les deux grandes crues d'octobre 1993 et de janvier 1994 qui participent à la modification du peuplement.

Il est intéressant de remarquer que l'année 2003, caractérisée par une longue période de canicule, s'inscrit dans la tendance établie sur tous les sites.

Un autre enseignement de cette étude, également visible sur la figure 20, réside dans le fait que les mêmes dérives temporelles sont observées en amont et en aval des CNPE. La modification du peuplement de poissons s'explique par l'effet prépondérant du changement climatique qui tend à masquer l'influence des rejets thermiques. La même étude réalisée sur les données des CNPE de Nogent-sur-Seine, de Belleville et de Chinon sur la Loire, aboutit aux mêmes conclusions (Daufresne \& Boët, 2005).

Pour mettre en évidence l'influence des rejets thermiques des CNPE, plusieurs études ont été réalisées sur le site de Bugey.

\subsubsection{Effet des rejets thermiques du CNPE du Bugey}

Le site de Bugey présente plusieurs particularités intéressantes pour mettre en évidence l'effet des rejets thermiques:

- il est situé sur le Haut-Rhône dans la partie la plus naturelle du fleuve,

- le CNPE comporte deux tranches en circuit ouvert qui produisent des rejets thermiques importants, les échauffements aux stations situées en aval en rive droite étant en permanence compris en moyenne entre $+5^{\circ} \mathrm{C}$ et $+8^{\circ} \mathrm{C}$ (Tab. XVII),

- l'échantillonnage des poissons se fait selon une procédure standardisée inchangée depuis 1979, en des stations situées à l'aval immédiat du rejet en rive droite soumises à un échauffement permanent significatif plus élevé que l'échauffement à l'aval éloigné du site.

Une première étude a porté sur les données collectées entre 1979 et 1994 (Ginot et al., 1996). En fonction de la température relevée au moment des pêches, trois « groupes thermiques » ont été distingués.

1. Groupe des températures de $7^{\circ} \mathrm{C}$ à $24^{\circ} \mathrm{C}$.

Ce groupe central comporte huit espèces dominantes qui constituent $96 \%$ de l'effectif total : 
Tableau XVII. Échauffement aux stations de pêches en aval du CNPE de Bugey par rapport aux températures relevées aux stations en amont. Intervalle des moyennes saisonnières sur la période 1979-1994 (Cattanéo et al., 2001), intervalle des mesures effectuées en 2001 (Roger \& Capra, 2002). La station 4 a été prospectée jusqu'en 1988.

Table XVII. Temperature rise at sampling sites downstream of Bugey NPP compared to the temperature recorded upstream. Range of seasonal means for the 1979-1994 period (Cattanéo et al., 2001), range of measurements in 2001 (Roger \& Capra, 2002). Station 4 was studied up to 1988 .

\begin{tabular}{cccc}
\hline Stations & \multicolumn{3}{c}{ Echauffement $\left({ }^{\circ} \mathrm{C}\right)$} \\
\cline { 2 - 4 } & $\frac{1979-1994}{\text { intervalle }}$ & intervalle & Moyenne \\
\hline 4 (rejet) & 9,2 à 11,4 & & \\
5 (proche rejet) & 6,5 à 8,2 & $7,5-9,4$ & 8,0 \\
$6 D$ & 3,7 à 5,9 & $1,4-7,7$ & 5,4 \\
7 & 2,6 à 3,6 & & \\
8 & 0,9 à 1,8 & & 1,1 \\
$6 G$ & 0,5 & $0,4-2,1$ & \\
\hline
\end{tabular}

\section{Groupes thermiques Bugey 1979-1984}

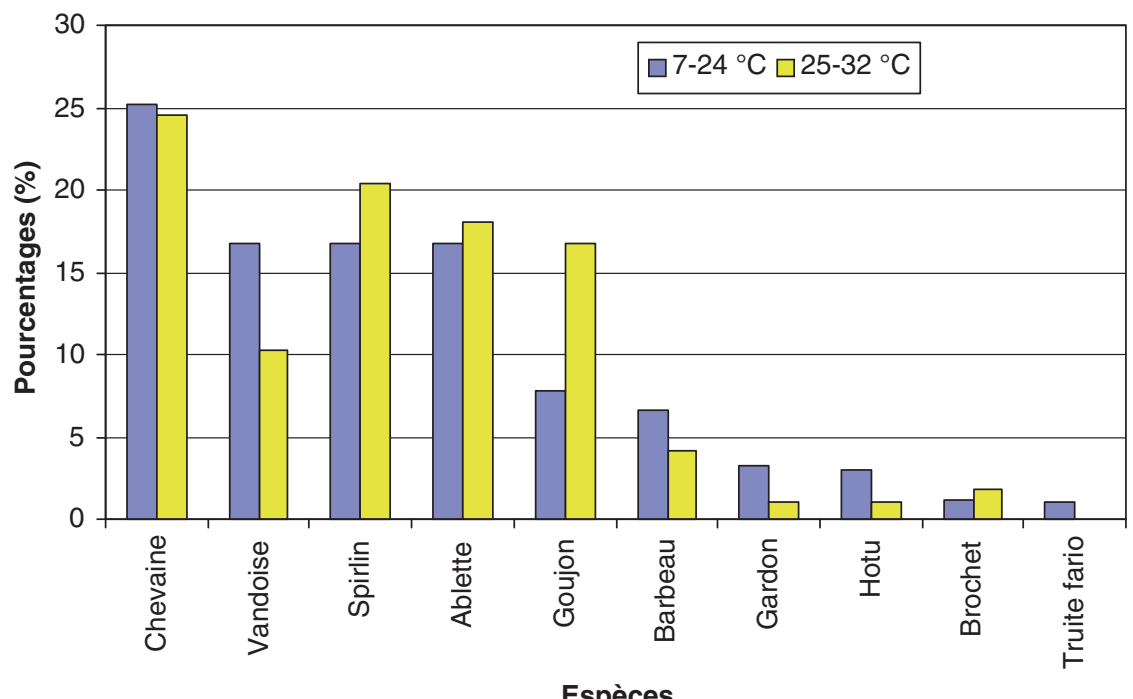

Fig. 21. Effectifs des espèces de poissons pêchés sur le site de Bugey dans deux groupes thermiques (température relevée au moment de la pêche) (Ginot et al., 1996).

Fig. 21. Relative abundance of fish caught in the Bugey study area for two thermal groups (temperature conditions at the time of sampling) (Ginot et al., 1996). 
chevaine, vandoise, spirlin, ablette, goujon, barbeau, gardon, hotu. La vandoise est mieux représentée que le spirlin. La truite fario y est très peu représentée (Fig. 21).

2. Groupe des températures de $25^{\circ} \mathrm{C}$ à $32{ }^{\circ} \mathrm{C}$.

II a été identifié dans les pêches d'été aux stations échauffées, le cortège des huit espèces dominantes représente $96 \%$ de l'effectif total. La vandoise est moins représentée que le spirlin et la truite fario est absente (Fig. 21).

3. Groupe des températures $<7^{\circ} \mathrm{C}$.

II rassemble les pêches hivernales et comporte les mêmes espèces dominantes avec une meilleure représentation de la vandoise $(45 \%)$ et de la truite fario (4\%).

Une étude statistique par AFC a été effectuée sur les données relatives au CNPE de Bugey de 1979 à 2004 pour rechercher l'impact local des rejets thermiques (Daufresne et al., 2005). La variance inter-stationnelle est trois fois plus basse que la variance interannuelle. L'analyse est de ce fait entachée d'incertitude mais elle permet toutefois de discriminer les deux groupes de stations : échauffées et non échauffées. Parmi les stations non échauffées se trouve la station 6G, en aval du CNPE mais en rive gauche; l'échauffement y est faible et intermittent selon le débit du fleuve et le fonctionnement des tranches en circuit ouvert.

Sur l'ensemble des données, le même noyau des huit espèces dominantes que celui identifié sur les données de 1979 à 1994 est retrouvé. Par rapport aux stations non échauffées, les stations échauffées en permanence de $+5^{\circ} \mathrm{C}$ à $+8^{\circ} \mathrm{C}$ en moyenne se caractérisent par :

- une proportion plus élevée de spirlins et d'ablettes parmi les espèces dominantes,

- une proportion plus élevée de blageons, de brèmes bordelières, de silures parmi les espèces marginales.

Les stations non échauffées conservent plus de truites, d'épinoches, et de loches franches parmi les espèces marginales.

Cette étude permet de conclure que les écarts entre stations échauffées et non échauffées vont globalement dans le même sens que les effets du changement climatique.

\subsubsection{Effet des facteurs hydrologique} et thermique sur la reproduction des Cyprinidés dans le Bas-Rhône

Olivier et Carrel (2006) ont analysé les données relatives aux Cyprinidés juvéniles collectées de 1982 à 2004 sur les trois sites situés en aval de Lyon : Saint-Alban, Cruas et Tricastin, en vue de dégager des tendances dans les séries chronologiques et de rechercher des liens avec les variables hydrologiques et thermiques.

Les données piscicoles sont restreintes aux vingt espèces de cyprinidés capturées sur le Bas-Rhône et les cohortes des juvéniles nés dans l'année en cours (poissons d'âge $0^{+}$). Le succès interannuel de la reproduction est estimé à partir des captures de fin d'été de cette classe d'âge.

L'analyse des données hydrologiques et thermiques montre d'une part le synchronisme de variation de ces facteurs sur les différents sites et 
d'autre part le lien étroit entre hydrologie et thermie, en particulier l'effet des débits élevés (moyens ou de crues). Ces derniers se traduisent par un retard dans l'échauffement de l'eau au printemps ou même par un écart à la courbe de référence inter-annuelle.

L'analyse des données biologiques montre le rôle prépondérant de huit espèces : gardon, ablette, chevaine, hotu, goujon, brème bordelière, bouvière et barbeau fluviatile, l'extrême variabilité interannuelle du recrutement au cours de cette chronique, important chez de nombreuses espèces pour les années 1986, 1989, puis à partir de 1994 sur la plupart des sites.

L'effet intersites n'est pas significatif, ce qui signifie que l'essentiel de la variabilité est d'origine temporelle et intéresse l'ensemble du Bas-Rhône. Une augmentation très nette de la richesse spécifique apparaît après 1993 puis se stabilise, à l'exception de l'année 1999 dans les sites aval.

Les données mettent également en évidence une alternance entre un peuplement dominé par des espèces lénitophiles (dont la plus représentative est le gardon) et un peuplement dans lequel la proportion d'espèces rhéophiles augmente (hotu, blageon, spirlin, barbeau). Le phénomène s'accentue à compter de 1994 sur les deux secteurs aval sur le Bas-Rhône. Un changement d'état du système entre 1993 et 1994 fait suite aux crues majeures survenues au cours de l'hiver 1993-1994. Elles constituent un épisode bref mais remarquable, un « reset event » postérieur à l'aménagement industriel de la vallée du Rhône, ayant entraîné de profonds bouleversements des dépôts sédimentaires et de la charge alluviale, un important nettoyage du substrat.
Des débits relativement faibles et stables pendant le printemps et l'été s'avèrent favorables à la reproduction des Cyprinidés. Des crues survenant pendant la fraie, l'éclosion et les premiers jours suivant l'apparition des larves, peuvent en revanche avoir un effet négatif sur le recrutement. L'ablette, espèce à ponte multiple, s'accommode mieux que d'autres espèces des fluctuations importantes de débits.

L'analyse conjointe de la variabilité thermique, hydrologique et biologique sur les sites du Bas-Rhône montre que la tendance à l'augmentation de la température printanière et estivale s'accompagne d'une augmentation du recrutement de la majorité des espèces de Cyprinidés. Elle met en évidence une structure temporelle comportant trois ensembles d'années :

- les années «froides q qui regroupent la période 1982-1988 et les années 1992, 1995, 1999 et 2001,

- les années plus chaudes dont les seuils thermiques à $12^{\circ} \mathrm{C}$ et $18^{\circ} \mathrm{C}$ sont plus précoces,

- les années 2003 et 2004 marquées par des températures estivales particulièrement élevées.

Le succès de la reproduction des cyprinidés, favorisé à la fois par des températures printanières et estivales élevées, par la prolongation des périodes chaudes, par la relative stabilité hydrologique et par la modicité des débits instaurées dans un contexte «chaud», ne doit pas laisser présumer une augmentation parallèle du recrutement parmi les autres classes d'âge. En effet, l'analyse effectuée ne prend pas en compte les poissons ayant franchi le cap du premier hiver. 
Ces résultats ne donnent donc aucune indication sur la relation entre le succès de reproduction et le recrutement dans les populations.

\subsubsection{Conclusions}

De l'analyse globale effectuée sur les peuplements de poissons des quatre sites de CNPE pendant la période de 25 ans entre 1979 et 2004 , quelques conclusions ressortent.

1. Une évolution similaire est observée sur tous les sites : les espèces d'eau froide régressent au profit des espèces thermophiles, elle est particulièrement nette à $\mathrm{Bu}$ gey et est interprétée comme une réponse au changement climatique manifesté par l'augmentation de la température de l'eau.

2. Le peuplement de poissons du Rhône évolue aussi sous l'effet de l'introduction de nouvelles espèces qui peuvent être favorisées par la dérive climatique.

3. L'effet du changement climatique est prépondérant sur l'effet des rejets thermiques des CNPE dans la mesure où la même tendance est observée sur les stations en amont et en aval des CNPE. L'effet des rejets thermiques intervient en second ordre mais il est toutefois décelable localement sur le site de Bugey dans les stations qui subissent un échauffement permanent et supérieur à l'échauffement après mélange $\left(+3^{\circ} \mathrm{C}\right.$ à $\left.+9^{\circ} \mathrm{C}\right)$. II se manifeste par une plus forte représentation des espèces thermophiles en ces stations.

4. Sur le Bas Rhône, le réchauffement précoce de l'eau au printemps et les températures printanières et estivales élevées favorisent la reproduction des Cyprinidés lorsque le débit reste relativement faible et stable.

5. L'hydrologie influe dans le BasRhône sur la variabilité du régime thermique, mais les grandes crues intervenues pendant l'hiver 19931994 et celles qui ont suivies ont nettement marqué l'évolution ultérieure du recrutement en favorisant à nouveau des espèces plus rhéophiles et lithophiles, entraînant ainsi une élévation de la richesse spécifique du peuplement de poissons.

6. La réintroduction des grands migrateurs dans le Rhône est un des objectifs de gestion prioritaires; la migration peut être perturbée au printemps par des variations thermiques de grande amplitude.

\section{QUELQUES CONCLUSIONS ET RECOMMANDATIONS}

De par ses aménagements hydroélectriques et ses centrales thermiques nucléaires, le Rhône est aujourd'hui un des grands fleuves européens équipés pour la production d'énergie. Les quatre CNPE actuellement en service sur le fleuve produisent près du quart de l'électricité nucléaire française. Cette capacité de production doit rester compatible avec les objectifs d'atteinte du bon état ou du bon potentiel écologique fixé par la DCE à l'horizon 2015. II était donc indiqué de donner une vision d'ensemble sur les rejets thermiques de ces installations non seulement sous l'aspect des échauffements cumulés mais aussi sur leur impact hydrobiologique. 


\subsection{Les invariants : l'aménagement énergétique du fleuve Rhône}

De profondes modifications morphologiques, hydrologiques, sédimentologiques sont intervenues dans le fleuve au cours du siècle dernier, elles jouent un rôle déterminant dans la structuration des peuplements aquatiques. Les composantes biologiques sont très liées aux conditions d'habitat originelles du fleuve, plus ou moins modifiées par les aménagements. Ainsi, parmi les secteurs étudiés, celui de Bugey a été peu transformé morphologiquement, alors que celui de Saint-Alban l'a été assez radicalement. II faut aussi mentionner que la résistance du peuplement résiduel tient beaucoup au maintien et parfois à la réhabilitation des tronçons du Rhône court-circuité (RCC), qui sont constitués par l'ancien lit du fleuve (près de $150 \mathrm{~km}$ cumulés) : ces tronçons servent de zones de refuge, de reproduction et de vie à la plupart des espèces du Rhône.

II existe par ailleurs, comme dans tout cours d'eau, un gradient longitudinal naturel des conditions hydrologiques, morphologiques et de température, qui influence fortement la répartition des communautés de macro-invertébrés et de poissons; ce gradient est très spécifique sur le Rhône avec plusieurs ruptures très nettes marquées par des apports périphériques : influence lacustre du Léman, influence glacio-nivale de l'Arve, influence de deux rivières pluviales (l'Ain et la Saône), influence nivale de l'Isère et enfin de l'Ardèche et de la Durance; il est nécessaire de garder à l'esprit cette succession pour bien interpréter toute tendance observée.

Les apports thermiques provenant des CNPE, qui ont été évalués dans le cadre des phases 1 et 2, induisent des échauffements résiduels moyens qui atteignent $+0,6^{\circ} \mathrm{C}$ à $+1,4^{\circ} \mathrm{C}$ de l'amont de Saint-Alban à l'amont d'Aramon. À l'aval immédiat des rejets thermiques, avant mélange complet des eaux, l'échauffement est beaucoup plus élevé : il atteint par exemple parfois $+9^{\circ} \mathrm{C}$ en rive droite en aval du CNPE de Bugey, la rive gauche n'étant pas échauffée.

\subsection{Les changements intervenus au cours des 25 dernières années}

Depuis la fin des années 1970, l'hydrosystème rhodanien évolue sous l'action conjuguée de plusieurs facteurs dont les effets sont appréhendés grâce notamment aux programmes de surveillance hydro-écologique des CNPE.

Une liste des principales causes des changements identifiés sur les vingt-cinq dernières années a été dressée :

- la dérive de la température de l'eau sous l'effet du changement climatique en cours a entraîné un échauffement moyen d'environ $+1,5^{\circ} \mathrm{C}$ sur le Haut-Rhône et $+3,0^{\circ} \mathrm{C}$ sur le Bas-Rhône,

- les évènements hydrologiques tels que les crues exceptionnelles de l'hiver 1993-1994 ou les étiages prolongés,

- la réduction de la pollution organique grâce aux efforts consentis pour le traitement de la pollution urbaine et qui a permis le maintien d'un niveau d'oxygénation satisfaisant même pendant l'épisode de canicule en 2003 ,

- la réduction de certaines pollutions potentiellement toxiques, mais la présence de polluants persistants 
tels que les PCB dans les sédiments,

- le développement d'espèces exogènes, particulièrement depuis 1990,

- la réalisation de nouveaux aménagements pendant la durée des suivis hydrobiologiques tels que la retenue de Sault-Brénaz (1986) en amont du CNPE de Bugey.

\subsection{Les réponses biologiques aux changements}

Confrontées à ces changements, les composantes biologiques évoluent par des modifications structurelles ou disparaissent des secteurs; les tendances ont été caractérisées dans cette étude par plusieurs traitements statistiques. Pour le facteur thermique, les analyses statistiques sont fondées sur des données:

- acquises au pas de temps horaire,

- moyennées à la journée pour les études statistiques, notamment lors des phases 1 et 2 de l'étude,

- agrégées à des pas de temps supérieurs (hebdomadaire à plurimensuel) pour l'évaluation de leur impact écologique.

En effet, les données collectées dans le cadre des suivis hydrobiologiques et piscicoles des CNPE sont acquises dans le but de mettre en évidence des modifications structurales au sein des peuplements et de les suivre sur le moyen ou le long terme, les peuplements étant considérés comme des intégrateurs des modifications à ces échelles de temps.

En réponse à l'élévation de la température sous l'effet du changement climatique, une modification générale dans la structure des peuplements de macro-invertébrés et de poissons a été observée sur le Haut-Rhône au cours des 25 dernières années et particulièrement au cours de la dernière décennie : les espèces thermophiles et lénitophiles ont été favorisées alors que les espèces sténothermes d'eau froide et rhéophiles ont montré une tendance à la régression (Daufresne et al., 2004, 2005). Une précocité de la maturation sexuelle et de la reproduction et l'arrêt de croissance estivale lorsque le seuil thermique supérieur est dépassé peuvent expliquer la régression des espèces sténothermes d'eau froide (Olivier \& Carrel, 2006). Ces évolutions sont observées localement, plus nettement aux stations en aval du site de Bugey qui sont exposées en permanence à des échauffements plus élevés (environ + $5^{\circ} \mathrm{C}$ et $+9^{\circ} \mathrm{C}$ ), que dans les stations situées en aval après mélange des eaux et homogénéité thermique.

Sur le Bas-Rhône, où les peuplements étaient déjà dominés par des taxons thermophiles et lénitophiles, cette dérive a été moins marquée (Bady \& Fruget, 2006; Daufresne \& Boët, 2005). Certaines espèces de Cyprinidés euryèces, comme le gardon, bénéficient des printemps et des étés plus chauds qui favorisent leur reproduction (Olivier \& Carrel, 2006) et leur survie (Souchon et al., 2005) dans les schémas thermiques actuellement observés. L'extension de la période chaude favorise les espèces de poissons dont la ponte est étalée ou peut être fractionnée. Peu de données existent sur les macrophytes en dehors de la surveillance effectuée à Saint-Alban; sur ce site la diversité des macrophytes semble peu affectée par un échauffement permanent voisin 
de $+5^{\circ} \mathrm{C}$. Une réduction de leur variété et de leur abondance est toutefois observée sur une partie de la zone en aval du CNPE.

Ces résultats sont en accord avec les évolutions potentielles des peuplements des cours d'eau français prévues par les modélisations réalisées dans le programme GICC-Aquabio (Pont, 2003). Sous l'effet de l'élévation de la température, cette étude prévoit pour les poissons une raréfaction des espèces actuellement caractéristiques des zones amont des bassins et un repli des espèces de Cyprinidés les plus rhéophiles vers ces zones amont.

Parmi les autres causes d'évolution, la qualité de l'eau qui s'est améliorée pour ce qui concerne les indicateurs de pollution organique, le décolmatage des fonds et/ou l'amélioration de la qualité physico-chimique du sédiment dans le Bas-Rhône lors des grandes crues de 1993-1994, ont constitué des facteurs favorables pour les macro-invertébrés et les poissons. Toutefois, ces remaniements du substrat sont à l'origine d'une remise en suspension de polluants toxiques accumulés de longue date (problème actuel des PCB).

Des espèces exotiques de macroinvertébrés et de poissons se sont installées dans le Rhône au cours du XX ${ }^{e}$ siècle, ces introductions apparaissent plus nombreuses depuis 1990. Certaines de ces espèces ont un caractère envahissant et sont susceptibles de modifier la structure des peuplements et d'avoir des impacts d'ordre fonctionnel.

Quoiqu'il en soit, il persiste un « gradient longitudinal » qui se manifeste par une meilleure qualité biologique du fleuve à l'amont de Lyon qu'à l'aval.
En dehors du chenal et des canaux d'amenée et de fuite des usines hydroélectriques, les tronçons courtcircuités et les annexes hydrauliques offrent des conditions d'habitats diversifiés propices à des espèces de plantes, d'invertébrés, de poissons. Ils constituent notamment des milieux privilégiés pour la reproduction des poissons. Ce sont aussi des refuges où subsistent des espèces protégées.

\subsection{Quelle est la part des rejets thermiques des CNPE dans ce contexte changeant?}

Dans les changements biologiques, les rejets thermiques interviennent en second ordre par rapport à l'évolution climatique et ils ont été observés surtout dans le chenal.

Le choix, au moment de la construction des CNPE, de ne pas mélanger immédiatement le rejet aux eaux du Rhône était motivé par la volonté d'éviter de créer une discontinuité thermique sur toute la largeur du fleuve qui aurait risqué de perturber le déplacement des poissons. De fait, les effets biologiques des rejets thermiques sont bien identifiés localement, à l'aval immédiat des CNPE, en des stations où l'échauffement est plus élevé (rive exposée au rejet) mais il subsiste en contrepartie des zones non échauffées sur la rive opposée.

Bien que la charge thermique appliquée au Rhône depuis une quinzaine d'année contribue à la modification écologique du fleuve, elle n'a pas eu d'effet majeur comparativement aux autres pressions anthropiques ou naturelles (climatiques). Par ailleurs, les 
tronçons court-circuités sont moins influencés par les rejets thermiques des CNPE que l'axe fluvial lui-même. Dans l'avenir, en absence de toute nouvelle installation de centrale thermique en circuit ouvert, la charge thermique restera constante et la part relative des rejets des CNPE par rapport à l'évolution naturelle prévue sous l'effet du changement climatique devrait même diminuer.

S'agissant des limites réglementaires de température et d'échauffement, jusqu'alors respectées en valeur instantanée, elles pourraient être établies selon d'autres modalités : le lissage journalier, sous réserve que les variations autour des seuils restent dans la gamme des amplitudes journalières observées sur le fleuve, pourrait ainsi être envisageable.

II faut enfin noter que, non comptés les effets sur la morphodynamique et l'habitat, le facteur hydrologique interagit avec les facteurs thermiques et physico-chimiques : les périodes de bas débits sont caractérisées par une moindre dilution des rejets thermiques, une plus forte amplitude journalière en température de l'eau (les échanges avec l'air étant facilités) et une plus forte amplitude des cycles liés à la photosynthèse sur l'oxygène dissous et le $\mathrm{pH}$. A contrario, les périodes de hauts débits se traduisent par un lissage des cycles et une dilution plus importante des rejets thermiques.

\subsection{Comment concilier les rejets thermiques avec l'atteinte des objectifs de qualité écologique du fleuve Rhône?}

II s'agit de maintenir les rejets thermiques dans un domaine compatible avec les objectif de la DCE du bon état écologique ou du bon potentiel écologique en tenant compte du changement climatique dans la mesure où le référentiel du bon état et du bon potentiel écologique intégrera dans l'avenir les effets du changement climatique.

Dans le chenal et les canaux où s'effectue l'écoulement principal du fleuve, les échauffements résiduels moyens induits par les rejets thermiques des $\operatorname{CNPE}\left(+0,6^{\circ} \mathrm{C}\right.$ à $+1,4^{\circ} \mathrm{C}$ ) sont nettement inférieurs aux élévations moyennes de température mesurées sur 25 ans $\left(+1,5^{\circ} \mathrm{C}\right.$ à $\left.+3,0^{\circ} \mathrm{C}\right)$. Le régime thermique $\mathrm{du}$ Rhône est donc principalement affecté par le changement climatique en cours, d'autant que la charge thermique rejetée est de plus en plus fréquemment contrainte par la température amont et les bas débits : les deux effets ne sont ni additifs, ni indépendants, mais ils se conjuguent (résultats de l'étude Rhône phase 2 sur les échauffements en période de température élevée du fleuve).

La surveillance hydrobiologique à long terme indique que les modifications constatées sur les peuplements de macro-invertébrés et de poissons s'expliquent en premier lieu par les effets du changement climatique.

Le régime thermique des tronçons court-circuités et des annexes hydrauliques est en grande partie indépendant de la température du Rhône en période estivale. Dans les conditions actuelles, ces milieux à haute valeur écologique ne sont pas affectés par les rejets thermiques des CNPE.

Les rejets thermiques doivent également être compatibles avec la migration des poissons amphihalins 
dans le Bas-Rhône (Combe et al., 2001); pour cela des connaissances sur les conditions thermiques compatibles avec les migrations de l'alose feinte et des autres espèces prises en compte dans le plan d'action 20042008 (Comité de gestion des poissons migrateurs du bassin RMC, 2003) doivent être acquises par des études in situ lorsque cela est possible. Le succès enregistré au cours des dernières années dans la montée de l'alose jusqu'en Ardèche(2) par les écluses de navigation de la CNR indique que les températures du printemps et du début de l'été permettent actuellement la migration de cette espèce. II faut toutefois préciser les seuils thermiques maximum de migration, mais aussi connaître l'effet de variations de température en quelques jours pour toutes les espèces figurant dans le plan d'action.

Enfin, les fortes réductions de débit résultant de la gestion hydraulique de la chaîne des aménagements du Rhône ont un impact direct sur les composantes biologiques (Olivier \& Carrel, 2006). Elles interfèrent en outre avec les rejets thermiques en augmentant transitoirement l'échauffement. Elles diminuent également le volume des zones refuges pour la faune. Une action sur l'hydrologie du fleuve dans le cadre d'une gestion de la production intégrée a été proposée par la DIREN de Bassin RMC (2003).

(2) À l'heure actuelle, suite à la capture de jeunes individus dans la retenue de Montélimar en 2005, l'alose serait susceptible de franchir les 5 premiers ouvrages du Rhône : Vallabrègues, Avignon, Caderousse, Donzère-Mondragon et Montélimar.

\subsection{Poursuivre et améliorer la surveillance hydro-écologique des CNPE et acquérir des connaissances}

Le programme actuel de surveillance hydro-écologique doit être poursuivi, il doit être adapté à la détection des changements thermiques, hydrologiques, chimiques et biologiques.

Le recueil systématique de données physico-chimiques et biologiques au titre des programmes de surveillance hydro-écologique des CNPE a permis de constituer des séries chronologiques qui ont été largement exploitées dans le cadre de cette étude et dans plusieurs publications scientifiques. Il est nécessaire de poursuivre ces mesures, en premier lieu pour contrôler l'impact des CNPE dans un contexte changeant, mais aussi pour mettre les données collectées à la disposition des chercheurs. Un point mérite cependant d'être examiné à l'occasion de la révision de ces programmes : améliorer le niveau de détermination des macroinvertébrés (à l'espèce) afin de permettre une meilleure exploitation des données collectées dans le cadre de ces programmes.

En période de crise climatique, la persistance de conditions hydrologiques et/ou thermiques exceptionnelles conduit à déclencher un programme de surveillance renforcé tel que celui mis en place par la DIREN de Bassin RMC lors de la canicule de 2003. Le retour d'expérience de ce programme a mis en évidence le rôle important tenu par les mesures en continu de température, oxygène dissous, $\mathrm{pH}$ et conductivité électrique aux stations automatiques installées et gérées par EDF à l'amont et à 
l'aval des CNPE. II convient de souligner la nécessité d'assurer la qualité de ces mesures malgré les difficultés rencontrées pendant ces périodes critiques.

Les observations in situ des mortalités de poissons pratiquées par les gardes-pêche constituent un précieux instrument d'alerte qui pourrait être complété par la conservation des poissons en vue d'une expertise vétérinaire pour identifier, si possible, la cause de mortalité.

Par ailleurs, l'observation et la collecte sur les filtres des organismes entraînés dans les prises d'eau des centrales thermiques du Rhône peuvent fournir des données qualitatives et semi-quantitatives sur les mortalités de poissons, de certains macroinvertébrés (cas des bivalves récoltés à Tricastin en 2003), d'algues et de macrophytes dérivants.

En matière de recherches, l'analyse des séries de données existantes a mis en évidence des modalités de fonctionnement des populations, en particulier chez les poissons. Néanmoins, ces hypothèses, concernant notamment le rôle de l'hydrologie et de la thermie dans le déroulement et le succès de la reproduction des cyprinidés, pourraient être testées par des protocoles de recueil de données ciblés pendant la période printanière sur les premiers stades de développement. Chez les invertébrés, la modélisation de traits biologiques particulièrement reliés à la thermie et à l'hydraulique s'avérerait intéressante en fonction de différents scénarios d'évolution climatique et/ou de rejet des CNPE.

D'autre part, la gestion des situations " de crise » telle que celle rencontrée au cours de l'été 2003, nécessiterait de compléter les observations par l'acquisition de résultats de type écophysiologique afin de préciser les réponses biologiques aux différentes variations du régime thermique du Rhône et en particulier dans les tronçons court-circuités. L'objectif est de prendre en compte les facteurs hydrologique et thermique dont tout indique qu'ils ne peuvent être considérés séparément en tant que facteurs écologiques.

Dans cette perspective, une action de recherche visant à intégrer les connaissances acquises dans un modèle de dynamique des peuplements couplé à des modèles hydrodynamiques et thermiques (étude spatiale à maille de quelques $\mathrm{m}^{2}$ de l'évolution conjointe de la disponibilité en habitats et en température pour les espèces électives du secteur en fonction de scénarios de gestion thermique et hydraulique) serait utile sur les secteurs à enjeux et permettrait de tester de nouveaux scénarios de changements (Souchon et al., 2005).

\section{RÉFÉRENCES BIBLIOGRAPHIQUES}

Agence de l'Eau Rhône Méditerranée \& Corse, 2004. Annexes géographiques de l'état des lieux, Version du 2 juillet 2004, 107 p.

Agence de l'Eau Rhône Méditerranée \& Corse, 2005. État des lieux, Bassin du Rhône et de cours d'eau méditerranéens, caractérisation du district et registre des zones protégées. Version adoptée par le Comité de Bassin du 4 mars 2005, $330 \mathrm{p}$.

Babut M. \& Miege C., 2007. Contamination des poissons et des sédiments du Rhône par les polychlorobiphényles. Synthèse des données recueillies en 2005-2006, Rapport Cemagref, Préfecture du Rhône. Juin 2007, 37 p.

Bady P. \& Fruget J.-F., 2006. Étude thermique du Rhône, Synthèse des 
données sur les invertébrés, Rapport intermédiaire, Rapport étude thermique du Rhône, phase III, 28 p.

Carrel G., 2002. Prospecting for historical fish data from the Rhone River basin : a contribution to the assessment of reference condition. Arch. Hydrobiol. 155(2) : 273-290.

Carrel G., 2006a. Le régime thermique du fleuve, Variabilité temporelle 19772004, Rapport Cemagref Aix en Provence, Rapport étude thermique du Rhône, phase III, 13 p.

Carrel G., 2006b. Physico-chimie des eaux du Rhône 1985-2004, Évolution des paramètres classiques, Rapport Cemagref Aix en Provence, Rapport étude thermique du Rhône, phase III, $18 \mathrm{p}$.

Carrel G., Pont D. \& Rivier B., 1995. Variabilité temporelle des peuplements piscicoles dans la section médiane du Bas-Rhône. Bull. Fr. Pêche Piscic. 337/338/339 : 101-111.

Cattanéo F., Carrel G., Lamouroux N., Breil P., 2001. Relationship between hydrology and Cyprinid reproductive success in the Lower Rhone at Montélimar, France. Archiv. Hydrobiol. 151: 427-450.

CNR, 2004. La crue du Rhône de décembre 2003, Synthèse hydrologique, Rapport N ${ }^{\circ}$ I218-DIT-HY 04-149, septembre 2004.

Combe P.-M., Le Corre M., Menella J.-Y. \& Perrin J.-F., 2001. La restauration des grands migrateurs rhodaniens et méditerranéens : contribution des sciences biologiques et sociales, Colloque International Lyon-Fleuves, $7 \mathrm{p}$.

Comité de bassin Rhône Méditerranée \& Corse, 2004. État des lieux / Document de première consultation officielle adopté par le comité de bassin le 2 juillet 2004, $22 \mathrm{p}$.

Comité de gestion des poissons migrateurs du bassin RMC, 2003. Plan de gestion des poissons migrateurs du Bassin Rhône-Méditerranée et Corse, 2004-2008, 142 p.
Daufresne M. \& Boët P., 2005. Évolution temporelle de la structure des peuplements piscicoles de grands fleuves français, Rapport EDF, Cemagref, $19 \mathrm{p}$.

Daufresne M., Roger M.C., Capra H., Lamouroux N., 2004. Long-term changes within the invertebrate and fish communities of the Upper Rhone River : effects of climatic factors. Global Change Biology 10 : 124-140.

Daufresne M., Souchon Y., Villeneuve B. \& Capra H., 2005. Influence du rejet thermique de la centrale nucléaire de Bugey dans un contexte de réchauffement climatique, Rapport Cemagref Lyon, EDF R\&D, Rapport étude thermique du Rhône, phase III, 21 p.

Desaint B., 2004. Étude thermique du Rhône, Phase 2, Rapport EDF DTG, $54 \mathrm{p}$.

Dessaix J., Fruget J.F., Olivier J.M. \& Beffy J.L., 1995. Changes of the macroinvertebrate communities in the dammed and by-passed sections of the French Upper Rhône after its regulation. Regulated Rivers 10(2-4) : 245-279.

Dessaix J. \& Fruget J.F., 2008. Évolution des peuplements de crustacés du Rhône Moyen au cours des 20 dernières années, relation avec la variabilité hydroclimatique. Hydroécol. Appl. $16: 1-27$

DIREN de Bassin RMC, 2003. Rejets thermiques exceptionnels des centrales de production d'électricité du Rhône, Rapport de suivi des impacts et orientations, $33 \mathrm{p}$.

Fruget J.-F., 2003. Changements environnementaux; dérives écologiques et perspectives de restauration du Rhône français : bilan de 200 ans d'influences anthropiques. VertigO 4(3) : 1-40.

Fruget J.-F., 2006. Étude thermique du Rhône, Étude locale des effets des rejets : exemple amont-aval de SaintAlban, Rapport étude thermique du Rhône, phase III, 8 p. 
Ginot V., Souchon Y. \& Roger P., 1996. Impact de l'élévation artificielle de température induite par le fonctionnement du CNPE du Bugey (fleuve Rhône) sur les communautés de poissons. Hydroécol. Appl. 8(1-2) : 1-33.

IPCC. 2007. Climate Change 2007: Synthesis Report. IPCC Fourth Assessment Report, http ://www.ipcc.ch/ ipccreports/ar4-syr.htm.

Lauters F. \& Poirel A., 2001a. Description statistique des longues séries de mesure de température de l'eau sur le Rhône et ses grands affluents (période 1977-1999), EDF - Département Surveillance Eau \& Ouvrages, Branche Environnement, Grenoble.

Lauters F. \& Poirel A., 2001b. Description statistique des longues séries de mesure de température de l'eau sur le Rhône et ses grands affluents (période 1977-1999) - Annexes, EDF - Département Surveillance Eau \& Ouvrages, Branche Environnement, Grenoble.

Ministère de l'écologie et du développement durable, 2005. Circulaire DCE 2005/12, 17 p.

Moatar F. \& Gailhard J., 2006. Water temperature behaviour in the River Loire since 1976 and 1881, Comptes-rendus Geoscience.

Nicolas Y. \& Pont D., 1995. Importance d'annexes latérales artificielles pour le recrutement en juvéniles de poissons dans un fleuve aménagé, le Bas-Rhône. Bull. Fr. Pêche Piscic. 337/338/339 : 249-257.

Olivier J.-M. \& Carrel G., 2006. La variabilité hydro-climatique et son rôle sur la reproduction des poissons Cyprinidés, 1982-2004, Rapport Cemagref Aix en Provence, UMR CNRS 5023, Rapport étude thermique du Rhône, phase III, $20 \mathrm{p}$.

Poirel A., Carrel G. \& Olivier J.-M., 2001. Illustration de la complémentarité des chroniques environnementales dans l'étude d'un hydrosystème fluvial: régime thermique et peuplements piscicoles du Rhône, Actes du colloque "Gestion des Grands Fleuves », juin 2001, Lyon.

Poirel A., Lauters F. \& Desaint B., 2008. 1977-2006 : Trente années de mesures des températures de l'eau dans le Bassin du Rhône. Hydroécol. Appl. $16: 191-213$.

Pont D. (coordinateur), 2003. Programme GICC-AQUABIO, Conséquences potentielles du changement climatique sur les biocénoses aquatiques et riveraines françaises, Rapport Final, novembre 2003, 382 p.

Roger M.-C. \& Capra H., 2002. Surveillance hydrobiologique du site du Bugey en 2001, Rapport Cemagref, Groupement de Lyon, 25 p. + annexes.

Roger M.-C., Faessel B. \& Lafont M., 1990. Réactualisation des données hydrobiologiques du Rhône sur le site du Bugey, Rapport Cemagref Lyon, novembre 1990, $67 \mathrm{p}$.

Roger M.-C., Souchon Y., Capra H., Chandesris A. \& Roger P., 2004. Surveillance hydrobiologique du site du Bugey en 2003, Rapport Cemagref, Groupement de Lyon.

Roger M.-C., Souchon Y., Capra H., Chandesris A., Roger P. \& Villeneuve B., 2005. Surveillance hydrobiologique du site du Bugey en 2004, Rapport Cemagref, Groupement de Lyon.

Souchon Y., Villeneuve B. Daufresne M. Capra H., 2005. Principaux effets des rejets thermiques décelés sur les peuplements de poissons du fleuve Rhône, Rapport Cemagref Lyon, EDF DTG, Rapport étude thermique du Rhône, phase III, 14 p.

Usseglio-Polatera P., 1985. Évolution des peuplements de Trichoptères et d'Ephéméroptères du Rhône à Lyon (1959-1982) : résultats de piégeage lumineux, Thèse de Doctorat, Université de Lyon, Villeurbanne, 2 volumes, 259 p., 203 p. 


\section{Annexe : Localisation des stations du programme de surveillance hydro-écologique des CNPE du Rhône}

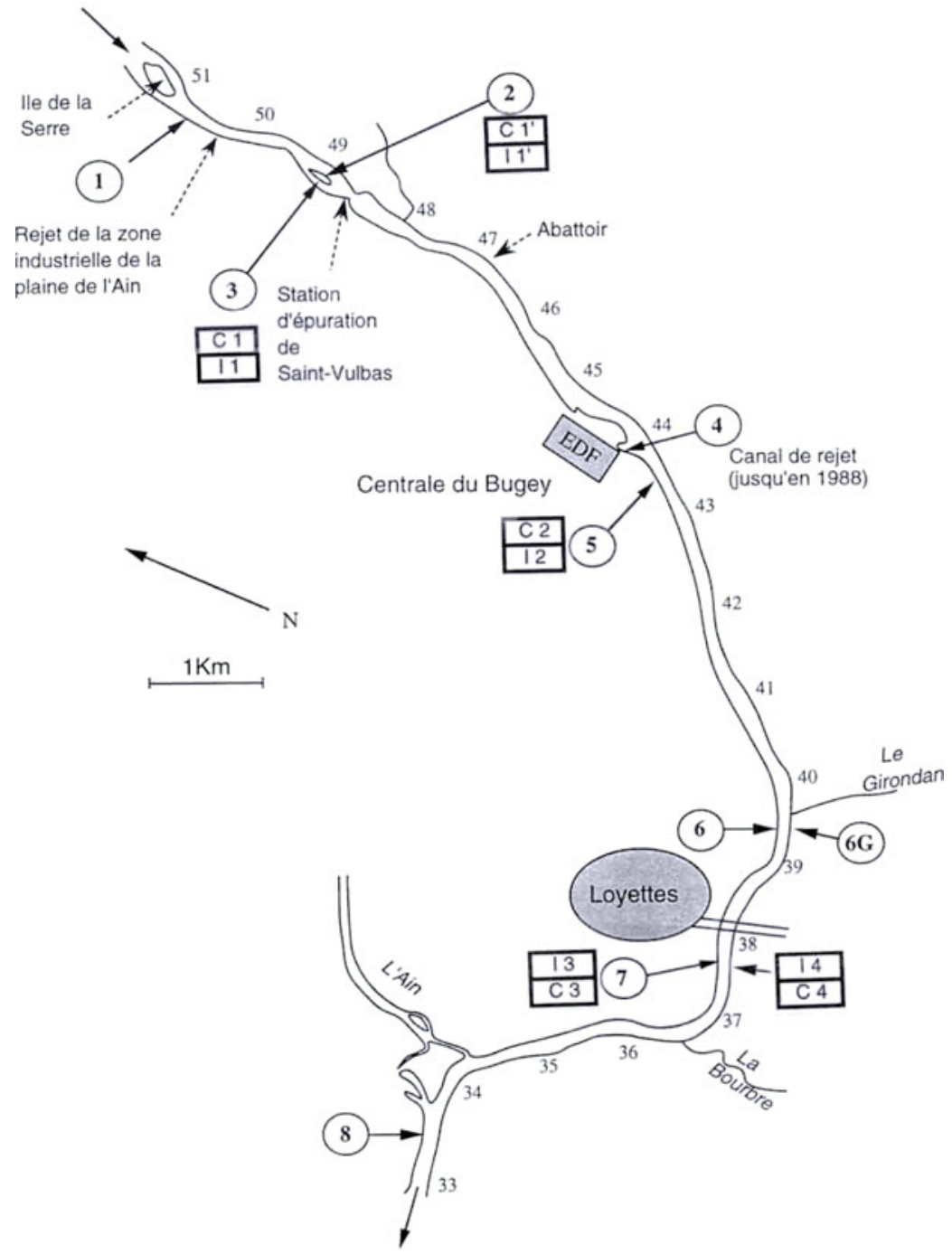

Fig. A1. CNPE de Bugey. Cercles : stations de pêches 1 à 8.11 à 14 : prélèvements d'invertébrés. C1 à $\mathrm{C} 4$ : analyses physico-chimiques.

Fig. A1. Bugey NPP. Circles: fish sampling points 1 to 8.11 to 14 : Invertebrates sampling points. C1 to C4 : physico-chemical measurements. 


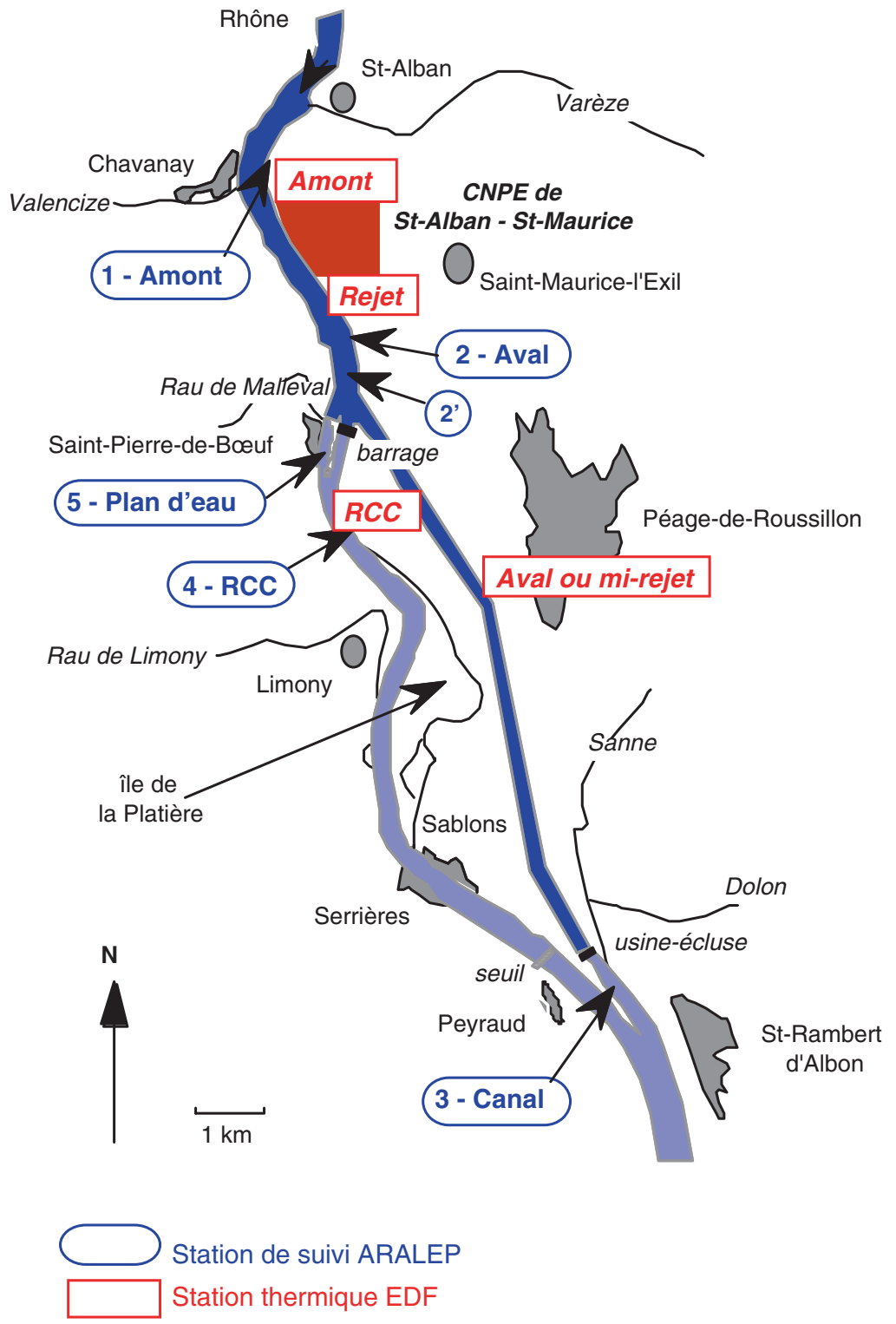

Fig. A2. CNPE de Saint-Alban. Localisation des stations de mesure du programme de surveillance hydro-écologique.

Fig. A2. Saint-Alban NPP. Location of sampling sites included in the hydro-ecological monitoring programme. 


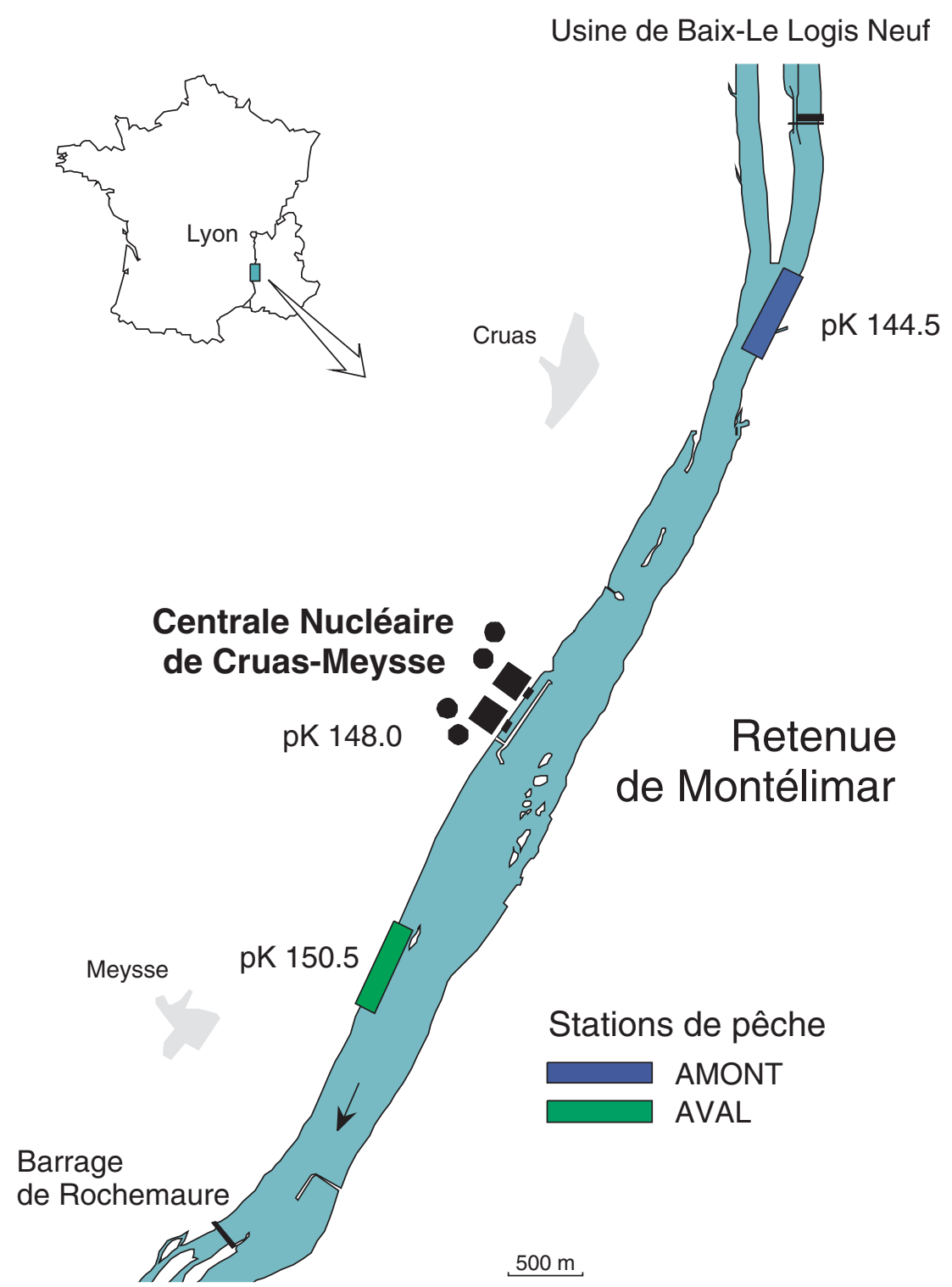

Fig. A3. CNPE de Cruas. Localisation des stations de pêche du programme de surveillance hydroécologique.

Fig. A3. Cruas NPP. Location of electrofishing sampling sites included in the hydro-ecological monitoring programme. 

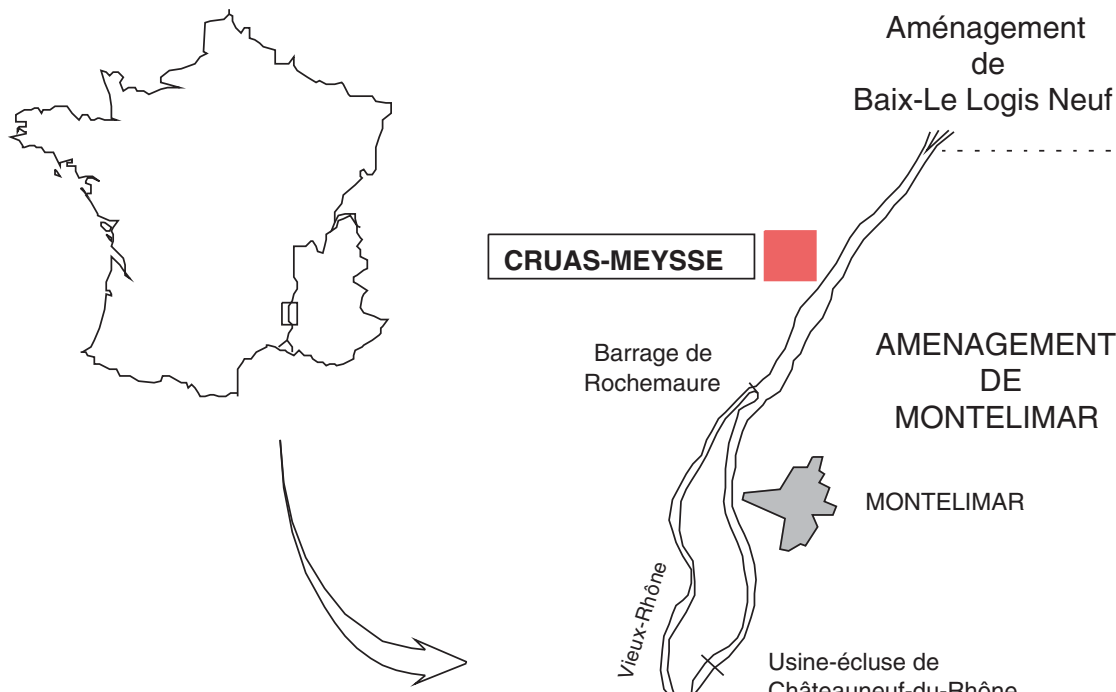

(1) Viviers

\section{CRUAS-MEYSSE}

Baix-Le Logis Neuf

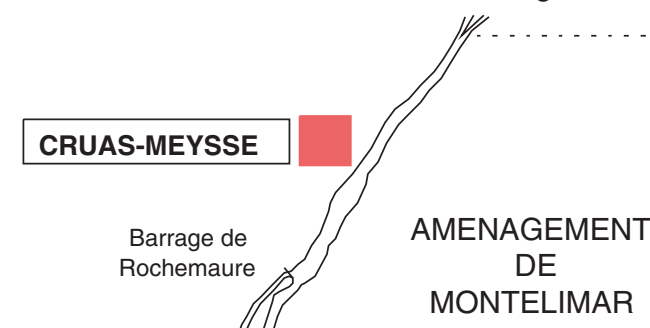

(2) Tricastin amont

(3) Tricastin rejet

(4) Tricastin aval

(5) Mornas

6) Pont-Saint-Esprit

Centrales nucléaires

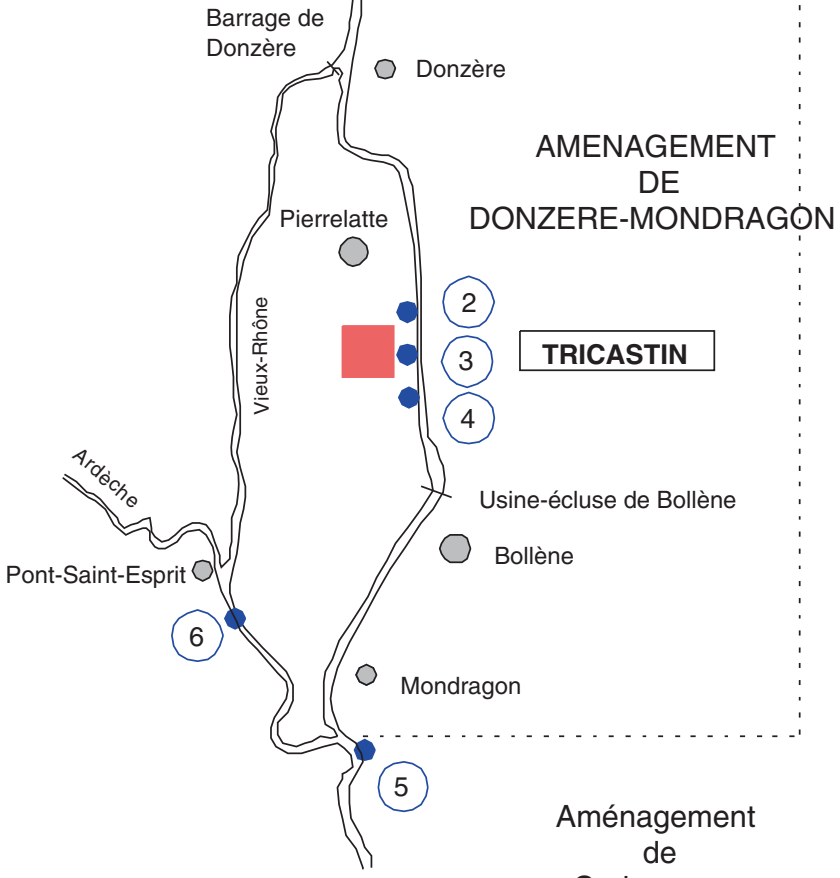

(6)

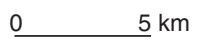

Caderousse

Fig. A4. CNPE de Tricastin. Localisation des stations de pêche du programme de surveillance hydroécologique.

Fig. A4. Tricastin NPP. Location of electrofishing sampling sites included in the hydro-ecological monitoring programme. 